\title{
XSTP: eXtended Satellite Transport Protocol
}

\author{
By \\ Maged E. Elaasar
}

\begin{abstract}
A thesis submitted to
the Faculty of Graduate Studies and Research

in partial fulfillment of

the requirements for the degree of

Master of Computer Science
\end{abstract}
Ottawa-Carleton Institute for Computer Science
School of Computer Science
Carleton University
Ottawa, Ontario

January 8, 2003

(c) Copyright

2002, Maged E. Elaasar 
National Library

of Canada

Acquisitions and Bibliographic Services

395 Wellington Street Ottawa ON K1A ON4 Canada
Bibliothèque nationale du Canada

Acquisitions et services bibliographiques

395, rue Wellington Ottawa ON K1A ON4 Canada
The author has granted a nonexclusive licence allowing the National Library of Canada to reproduce, loan, distribute or sell copies of this thesis in microform, paper or electronic formats.

The author retains ownership of the copyright in this thesis. Neither the thesis nor substantial extracts from it may be printed or otherwise reproduced without the author's permission.
L'auteur a accordé une licence non exclusive permettant à la Bibliothèque nationale du Canada de reproduire, prêter, distribuer ou vendre des copies de cette thèse sous la forme de microfiche/film, de reproduction sur papier ou sur format électronique.

L'auteur conserve la propriété du droit d'auteur qui protège cette thèse. $\mathrm{Ni}$ la thèse ni des extraits substantiels de celle-ci ne doivent être imprimés ou autrement reproduits sans son autorisation.

\section{Canadä}


The undersigned hereby recommend to the Faculty of Graduate Studies and Research acceptance of the thesis,

XSTP: eXtended Satellite Transport Protocol

submitted by

Maged E. Elaasar

In partial fulfillment of the requirements for the degree of

Master of Computer Science

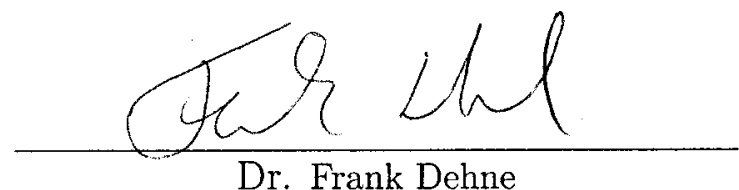

(Director, School of Computer Science)

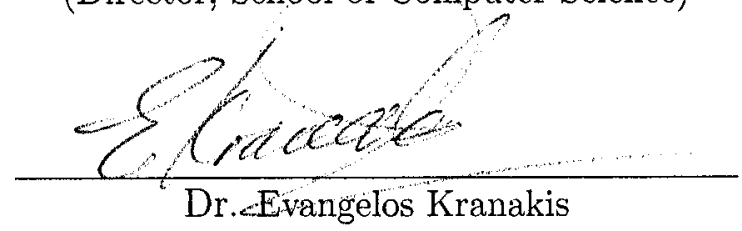

(Thesis Supervisor)

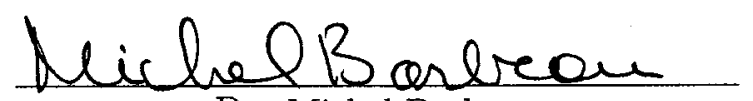

Dr. Michel Barbeau

(Thesis Supervisor)

Carleton University

January 8, 2003 


\section{Abstract}

The explosion of the e-community has spurred the need for a more global communication paradigm. Today, more people than ever are using mobile ad hoc networks to fulfill their communication needs. In particular, LEO satellite access networks are gaining popularity as an alternative or a complimentary way to get connected. Being both wireless and mobile, these networks have a unique set of link errors including bit corruption, handoff and limited connectivity. Unfortunately, most transport protocols, like the standard TCP, are only designed to handle congestion-related errors common in wired networks. This inability to handle multiple kinds of errors results in severe degradation in effective throughput and energy saving, which are relevant metrics for a mobile ad hoc environment. A recent study proposed a new transport protocol for satellites called STP that addressed many of the unique problems of satellite networks. However, there was no explicit attempt to implement a differentiating error control strategy in that protocol. This thesis proposes grafting a new probing mechanism in STP to make it more responsive to the prevailing error conditions in the network. The mechanism works by investing some time and transmission effort to deduce the cause of error. However, this overhead is recouped by handsome gains in both the connection's effective throughput and its energy efficiency. This sender-only mechanism leverages many unique features of STP, making it simple, scalable and easy to deploy. The mechanism's unique semantics make it quick to start and finish, yet efficient by its ability to self-terminate if it has prematurely started. Most importantly, the mechanism also preserves the end-to-end semantics of STP. The simulation compares the STP protocol both with and without the probing mechanism in different error conditions. The results show that this mechanism helps STP yield higher levels of throughput, while maintaining lower levels of overhead. The STP protocol with the new probing mechanism is called: the eXtended Satellite Transport Protocol (XSTP). 


\section{Acknowledgements}

Pursuing a master degree is a journey that one cannot make alone; and in this space I would like to thank the many people who have contributed to my successful journey.

First, I want to thank Allah for helping me out all along and enlightening my way. I also want to thank my wife Nariman, whose love, support and dedication throughout this whole journey has helped get me through it all. From putting up with long hours to helping me celebrate little victories along the way. I also thank my parents, whose prayers and support finally paid off.

Dr. Kranakis and Dr. Barbeau have been great supervisors. Dr. Kranakis gave me a lot of freedom in pursuing my research, while also providing wise advice and helpful discussions along the way. I have also been continually impressed with Dr. Barbeau's ability and dedication in leading a big group of students toward their degrees. He consistently inspired and motivated me to do my best. I feel very fortunate to have both of them as my advisors at Carleton.

I am also grateful to many other people including the members of my SmallSat group for their help and support. I also thank Waleed Ibrahim, Abdel-Rahman Amer and Hesham Elsayed for the enlightening technical discussions. Last but not least, I want to thank my employer Rational Software for funding my degree and putting up with my academic deadlines. 


\section{Table of Contents}

Abstract

Acknowledgements $\quad$ iv

List of Tables viii

List of Figures $\quad$ ix

List of Acronyms $\quad$ xi

1 Introduction $\quad 1$

1.1 Overview of LEO Satellite Networks . . . . . . . . . . . . . . . 3

1.1.1 Network Architecture . . . . . . . . . . . . . . . . 3

1.1 .2 Link Properties . . . . . . . . . . . . . . . . . . . . . 4

1.2 Transport over LEO Satellites . . . . . . . . . . . . . . . . . . 7

1.2.1 Unique Challenges . . . . . . . . . . . . . . . 7

1.2 .2 Error Control Strategies . . . . . . . . . . . . . . . . . . . . 9

1.2 .3 State of the Art . . . . . . . . . . . . . . . 10

1.3 Thesis Contribution . . . . . . . . . . . . . . . . . . . . 12

1.4 Thesis Outline . . . . . . . . . . . . . . . . 15

2 Background and Related Work 16

2.1 TCP Proposed Extensions for Satellites . . . . . . . . . . . . . . . . . 17

2.1.1 Control Parameter Proposals . . . . . . . . . . . . . . 18

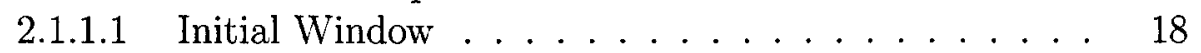

2.1.1.2 Maximum Segment Size . . . . . . . . . . . . 19

2.1.1.3 Receiver Window . . . . . . . . . . . . . . 20

2.1.1.4 Sender and Receiver Buffers ............ . . 21

2.1.1.5 Round Trip Time . . . . . . . . . . . . . 21

2.1.1.6 Connection Handshaking . . . . . . . . . . 22

2.1.2 Flow Control Proposals . . . . . . . . . . . . . . . . . . 24 
2.1.2.1 Byte Counting . . . . . . . . . . . . . 24

2.1.2.2 Transmission Pacing . . . . . . . . . . . . . 25

2.1.2.3 Disabling Delayed Acknowledgements . . . . . . . . 26

2.1.2.4 Selective Acknowledgements . . . . . . . . . . . 26

2.1.2.5 Acknowledgement Compression . . . . . . . . . 27

2.1 .3 Error Control Proposals . . . . . . . . . . . . . . . . 28

2.1.3.1 Proposed Classifications . . . . . . . . . . . 30

2.1.3.2 End-to-End Proposals . . . . . . . . . . . . . . 33

2.2 STP: Satellite Transport Protocol . . . . . . . . . . . . . . . 40

2.3 SCPS-TP: SCPS Transport Protocol . . . . . . . . . . . . 46

2.4 Summary $\ldots \ldots \ldots \ldots \ldots \ldots \ldots \ldots \ldots \ldots \ldots$

3 XSTP: Extended Satellite Transport Protocol 48

3.1 General Architecture . . . . . . . . . . . . . . . . . . . . 49

3.2 Detailed Design . . . . . . . . . . . . . . . . . . . 53

3.2 .1 Protocol Class . . . . . . . . . . . . . . . . . . 53

3.2 .2 Session Class . . . . . . . . . . . . . . . . 58

3.2 .3 Receiver Class . . . . . . . . . . . . . . . . . . . 62

3.2 .4 Sender Class . . . . . . . . . . . . . . . . . . . . 65

4 XSTP-Probing Mechanism $\quad 71$

4.1 Motivation . . . . . . . . . . . . . . . . . . . 71

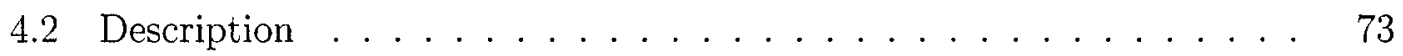

4.3 Design . . . . . . . . . . . . . . . . 84

5 Simulation and Performance Metrics 88

5.1 Simulation Environment . . . . . . . . . . . . . . . . . . . . 89

5.2 Simulation Configuration . . . . . . . . . . . . . . . 93

5.3 Performance Metrics . . . . . . . . . . . . . . . . . . 98

5.4 Test Cases . . . . . . . . . . . . . . . . . . . 100

6 Simulation Results and Analysis 104

6.1 Bit Corruption Tests . . . . . . . . . . . . . . . . . 105

6.1 .1 Effective Throughput . . . . . . . . . . . . . . 107

6.1 .2 Overhead .................... . . 110

6.1.3 Throughput/Overhead Ratio . . . . . . . . . . . . . . . . 113

6.2 Handoff Tests . . . . . . . . . . . . . . . . . . . . . . 113

6.2 .1 Effective Throughput . . . . . . . . . . . . . 116

6.2 .2 Overhead . . . . . . . . . . . . . . . 118

6.2 .3 Throughput/Overhead Ratio . . . . . . . . . . . . 120

6.3 Limited Connectivity Tests . . . . . . . . . . . . . . . . . . 122 
6.3.1 Effective Throughput . . . . . . . . . . . . . . . 124

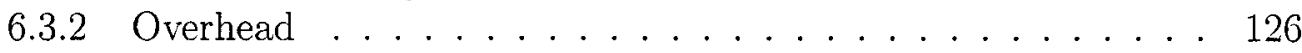

6.3.3 Throughput/Overhead Ratio............. 129

7 Conclusion and Future Work 131

$\begin{array}{ll}\text { Bibliography } & 137\end{array}$

A Transmission Control Protocol 143

$\begin{array}{ll}\text { B Measurement Data } & 150\end{array}$ 


\section{List of Tables}

B.1 Bit Corruption Test Measurements . . . . . . . . . . . . . . . 152

B.2 Handoff Test Measurements . . . . . . . . . . . . . . . 153

B.3 Limited Connectivity Test Measurements . . . . . . . . . . . . . . . . 154 


\section{List of Figures}

1.1 A typical satellite access network ............... 4

2.1 Error classification techniques for wireless networks [Gur00] . . . . . 32

2.2 Inter-arrival gap scheme $[$ BV99] . . . . . . . . . . . . . . . 35

2.3 Spike scheme $[\mathrm{CCV02}] \ldots \ldots \ldots \ldots \ldots$

2.4 Zigzag scheme $[$ CCV02] . . . . . . . . . . . . . . . . . 37

2.5 STP segment kinds $[$ Kat99] . . . . . . . . . . . . . . 42

3.1 A PIX message ... . . . . . . . . . . . . . . . 50

3.2 PIX protocol and session configurations including XSTP . . . . . 51

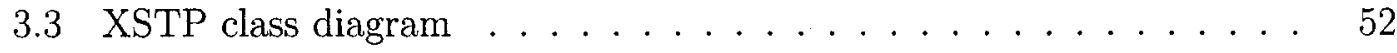

3.4 XSTP active and passive keys . . . . . . . . . . . 53

3.5 XSTP session connection sequence diagram . . . . . . . . . . 56

3.6 XSTP session disconnection sequence diagram . . . . . . . . . . . 57

3.7 XSTP session state machine . . . . . . . . . . . . . 61

4.1 A flow chart of XSTP probing mechanism . . . . . . . . . . . . 75

4.2 Breaking the POLL-STAT interleaf . . . . . . . . . . . . . 77

4.3 Triggering of the probing mechanism by an early timeout event . . . . 77

4.4 Triggering of the probing mechanism by a false early timeout event . 79

4.5 Phases of a probing cycle as it happens in the network . . . . . . . 81

4.6 Phases of a probing cycle as reflected in the probing map . . . . . . 81

5.1 Simulating a virtual network using QLP . . . . . . . . . . . . . 91

5.2 The two-state Markov chain in the XDELDROP protocol . . . . . . 93

5.3 The general simulation configuration . . . . . . . . . . . . . . 94

5.4 The four states of the simulated network . . . . . . . . . . . 97

5.5 The three simulated link errors . . . . . . . . . . . . . . . . 101

6.1 Effective throughput under bit corruption with 1 second mean duration 108

6.2 Effective throughput under bit corruption with 10 seconds mean duration 108

6.3 Overhead under bit corruption with 1 second mean duration . . . . 111 
6.4 Overhead under bit corruption with 10 seconds mean duration . . . . 111

6.5 Throughput/overhead ratio under bit corruption with 1 second mean duration . . . . . . . . . . . . . . . . . 114

6.6 Throughput/overhead ratio under bit corruption with 10 seconds mean duration . . . . . . . . . . . . . . . . . . 114

6.7 Effective throughput under handoff with 0.5 second rendezvous . . . . 117

6.8 Effective throughput under handoff with 1 second rendezvous . . . . . 117

6.9 Overhead under handoff with 0.5 second rendezvous . . . . . . . . . . 119

6.10 Overhead under handoff with 1 second rendezvous . . . . . . . . . . . 119

6.11 Throughput/overhead ratio under handoff with 0.5 second rendezvous 121

6.12 Throughput/Overhead ratio under handoff with 1 second rendezvous 121

6.13 Effective throughput under limited connectivity with 5 seconds rendezvous ........................... 125

6.14 Effective throughput under limited connectivity with 10 seconds rendezvous ........................... 125

6.15 Overhead under limited connectivity with 5 seconds rendezvous . . . 127

6.16 Overhead under limited connectivity with 10 seconds rendezvous . . . 127

6.17 Throughput/overhead ratio under limited connectivity with 5 seconds rendezvous . . . . . . . . . . . . . . . . 130

6.18 Throughput/overhead ratio under limited connectivity with 10 seconds rendezvous . . . . . . . . . . . . . . 130

A.1 A typical behavior of a TCP session [Gur00] . . . . . . . . . . . 147 


\title{
List of Acronyms
}

\author{
ACK Acknowledgment Flag \\ APP Application Protocol \\ ARQ Automatic Repeat Request \\ ATM Asynchronous Transfer Mode \\ BER Bit Error Rate \\ BGN Begin Flag \\ BGNAK Begin Acknowledgement Flag \\ CC Connection Count Option \\ CCECHO Connection Count Echo Option \\ CI Confidence Interval \\ DUPACK Duplicate Acknowledgment \\ END End Flag \\ ENDAK End Acknowledgment Flag \\ EST Established State \\ FIFO First In First Out Policy \\ FIN Finish Flag \\ FIN-ACK Finish Acknowledgment Flag \\ FTP File Transport Protocol \\ GEO Geostationary Earth Orbiting Satellite \\ ICMP Internet Control Message Protocol \\ IETF Internet Engineering Task Force \\ IP Internet Protocol \\ LEO Low Earth Orbiting Satellite \\ LIMD/H Linear Increase/Multiplicative Decrease with History \\ MSS Maximum Segment Size \\ MTU Maximum Transfer Unit \\ NACK Negative Acknowledgement
}




$\begin{array}{ll}\text { PEP } & \text { Performance Enhancing Proxy } \\ \text { PIX } & \text { Protocol Implementation framework for Linux } \\ \text { POLL } & \text { POLL Flag } \\ \text { POSIX } & \text { Portable Operating System Interface for X-based Systems } \\ \text { QLP } & \text { Queue Link Protocol } \\ \text { RFC } & \text { Request For Comment } \\ \text { ROTT } & \text { Relative One-Way Trip Time } \\ \text { RST } & \text { Reset Flag } \\ \text { RTO } & \text { Retransmission Timeout } \\ \text { RTT } & \text { Round Trip Time } \\ \text { SACK } & \text { Selective Acknowledgement } \\ \text { SCPS } & \text { Space Communication Protocol Specification } \\ \text { SCPS-TP } & \text { SCPS Transport Protocol } \\ \text { SD } & \text { Sequenced Data Segment } \\ \text { SD } & \text { Standard Deviation } \\ \text { SSCOP } & \text { Service Specific Connection Oriented Protocol } \\ \text { STAT } & \text { Status Flag } \\ \text { SYN } & \text { Synchronization Flag } \\ \text { SYN-ACK } & \text { Synchronization Acknowledgment Flag } \\ \text { T/TCP } & \text { TCP for Transactions } \\ \text { TCP } & \text { Transmission Control Protocol } \\ \text { UDP } & \text { User Datagram Protocol } \\ \text { STP } & \text { Satellite Transport Protocol } \\ \text { USTAT } & \text { Unsolicited Status Flag } \\ \text { VDELDROP } & \text { Virtual Delete and Drop Protocol } \\ \text { VSAT } & \text { Very Small Aperture Terminal } \\ \text { XDELDROP } & \text { Extended Delay and Drop Protocol } \\ \text { XSTP } & \text { Extended Transport Protocol } \\ \text { XSTP-OFF } & \text { XSTP with the probing option turned off } \\ \text { XSTP-ON } & \text { XSTP with the probing option turned on } \\ & \end{array}$




\section{Chapter 1}

\section{Introduction}

Communication has always been vital to the growth and development of human society. Earlier societies have always strived to come up with newer ways of communication. In today's information age, computer networks and the evolution of the Internet have transformed the world into one global village. However, the explosion of the e-community has spurred the need for a more global communication paradigm; one that will eventually ensure that connectivity is commonplace at home, at work or on the move. This requirement helped steer research in the wireless direction. Early attempts lead to the evolution of wireless data networks, where Internet users can communicate while roaming freely without the need of any wired setup. While these attempts were a large step in the right direction, they did not scale well for some applications that required quick deployment and automatic configuration. As a logical succession, attention moved to mobile ad hoc networks, where groups of mobile computing devices communicate among each other without the need of a fixed 
network setup.

Satellite data networks are a typical example of mobile ad hoc networks. Satellite networks have enabled a whole new class of applications by connecting telecommunication networks, empowering cellular networks, transmitting radio and video signals and collecting intelligence information. Satellites are generally divided into two types: Geostationary Earth Orbiting (GEO) and Low Earth Orbiting (LEO). However, throughout this paper the focus is on networks containing LEO satellite links, which have different characteristics from their traditional GEO counterparts. As detailed by Sun et al. [SSC96], LEO satellites are subject to much reduced transmission path loss and much shorter signal time delays. In addition, LEO satellites are relatively inexpensive, making them an economical solution for Internet connectivity in areas of the world otherwise handicapped by costly terrestrial infrastructure setup. LEO satellites are also perfect for experimentation, particularly during a time when research funds are running low and the possibility of failure is quite high.

While a lot of research has focused on the development of routing protocols for satellite networks, as exemplified in [RS96, CE95], the research in this thesis focuses mainly on enhancing the transport behavior over networks containing LEO satellite links. Although wireless and satellite links certainly share a lot of common characteristics, like high bit-error rates and intermittent connectivity, they also have enough 
distinct properties to be taken as different environments for data transport. As surveyed by SCPS [SCP98], these properties include highly variable round trip times, disproportional up and down link capacities, limited computing resources (power, memory and speed), and relatively higher throughput.

\subsection{Overview of LEO Satellite Networks}

\subsubsection{Network Architecture}

The typical architecture of LEO satellite networks exposes a different set of challenges to unaware transport protocols. Since a LEO satellite network is both ad hoc and mobile, its topology and configuration are generally random and frequently changing. Therefore, manual fine tuning of transport protocols, which is usually done by network administrators, is not enough in this case. As described by Sangal [San99], the general LEO satellite network infrastructure consists of three main components:

Terminals which are computing devices equipped with packet radio transceiver hardware, allowing them to communicate directly with satellites when they fall within their coverage area.

Base Stations which act as gateways between satellite and terrestrial switched networks. They also send signals for controlling satellite configuration.

LEO Satellites which are revolving around the earth in multiple paths and constellations. 


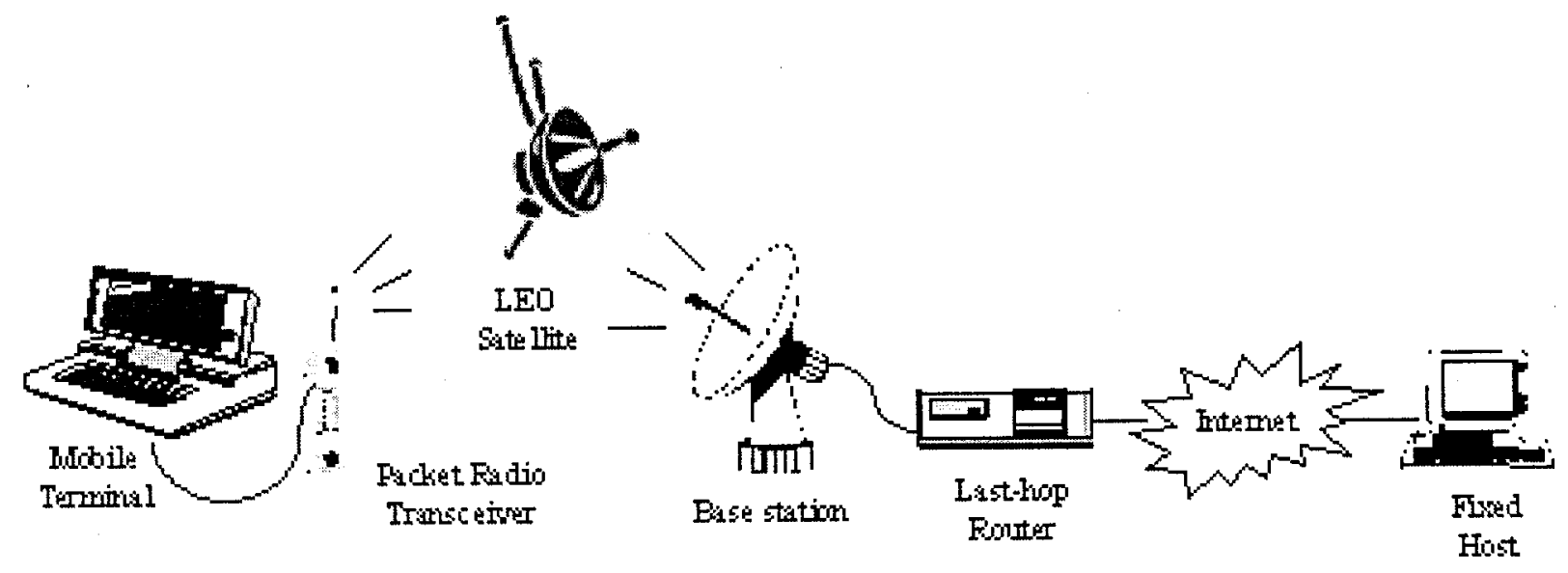

Figure 1.1: A typical satellite access network

LEO Satellite networks are often used as access points to other networks like the Internet. In this case, they are called LEO satellite access networks. For a fixed terminal in the Internet, a LEO satellite is visible as another Internet node on the IP layer. Moreover, it is also common for both ends of a network connection to be connected through either satellite--only or mixed satellite and other kind of links. A typical setup for a LEO satellite access network is depicted in Figure 1.1.

\subsubsection{Link Properties}

Since a satellite link is basically a special kind of wireless link, it naturally inherits all of a wireless link's characteristics. Allman et al. [AGS99, ADG $\left.{ }^{+} 00\right]$ and the researchers at SCPS [SCP98] describe satellite links as having a natural broadcast capability and an inherent ability to reach mobile users, which make satellite links 
ideal substitutes for terrestrial links. Satellite links also cover large areas resulting in reduced switching and forwarding overhead. However, being radio-based, satellite links can have limited bandwidth due to the natural limitation of the radio spectrum and due to certain international agreements that control the allocation of this resource. Also affected are transport protocols as satellite links have limited ability to trade bandwidth to solve other design problems.

Satellite networks are also characterized by having asymmetric links, usually due to the high cost of the needed technology to support both directions. This asymmetry is typically manifested in the links' speeds, bandwidth or both. It impacts the performance of transport protocols that use acknowledgements in the reverse direction as a self-clocking mechanism. Having slower-arriving acknowledgments hinders transmission speeds in the forward direction. Another restricting feature of LEO satellites is their limited computing resources largely due to power and size limitations. Power restrictions in particular can affect the stability of a connection and the frequency of errors, putting a damper on the overall performance of a transport protocol.

Similar to other wireless links, satellite links are lossy with potentially high biterror rates (BER), resulting in frequent packet drops. This effect is mainly due to several environmental factors (rain, pollution,etc) that cause noise, multi-path distortion and shadowing of radio channels. However, advanced error control coding is sometimes used in the link layer to mitigate such problems. It is still challenging 
though to hide the side effect of those solutions from unaware transport protocols. In fact, most reliable transport protocols have an inherent assumption that any loss is due to network congestion. Therefore, when a segment loss is detected, these transport protocols switch to a more conservative stance, effectively under-utilizing the link.

Another problem for LEO satellites is their intermittent connectivity due to their constant movement in their orbits. The window of connectivity to a given satellite is around 10\% of the time [SCP98]. A handoff occurs when one LEO satellite goes out of range and other one goes in range of some satellite access device. It can also happen when a LEO satellite leaves the range of some base-station and enters the range of another. The connectivity can also be lost for more extended periods due to a physical obstruction of the satellite signal or an inefficient distribution of the LEO satellites by the service provider. Both handoff and limited connectivity can lead to periods of "blackout", during which all packets get dropped. As mentioned by Allman et al. [AGS99], these periods are usually confusing for unaware transport protocols and may lead them to take wrong decisions (like invoking congestion control) that severely affect their performance. 


\subsection{Transport over LEO Satellites}

\subsubsection{Unique Challenges}

All data networks, including satellite access networks, are run by communication protocol stacks. The performance of any data connection is a direct reflection of the aggregate performance of all protocols in the stack. The transport protocol is one of the crucial protocols in any communication stack. While some transport protocols provide unreliable service, this thesis explores those that provide end-to-end reliable (complete, correct, in-sequence and without duplication) transmission service. Many standard transport protocols (like TCP) are generally unaware of the specific characteristics of their underlying networks. According to Tsaoussidis and Matta [TM01], these protocols are originally designed to address the problems and satisfy the transport goals of wired networks. Therefore, such protocols assume continuous connectivity, data loss resulting from network congestion and balanced bi-directional links. These protocols are also calibrated to overcome the problems of stability and heterogeneity in terms of receiver buffers, network bandwidth and delay. In addition, such protocols strive for fairness in bandwidth consumption and efficiency in link utilization by adopting proper congestion control mechanisms.

These features perform well for wired, high-speed networks. However, they fail miserably for satellite data networks, rendering their performance unacceptable. As explained by researchers at SCPS [SCP98], transport protocols targeted for satellite 
data networks should strive to provide fair link access, high aggregate throughput and high reliability through differentiating between unique error conditions. Transport protocols should also base bandwidth utilization on a precedence policy, maximize link utilization and provide an optional semi-reliable service when needed. The foregoing is in addition to providing new or updated algorithms to handle the unique properties of satellite networks mentioned earlier. These objectives call for revisiting the standard transport protocols and possibly proposing new ones. Specifically, Tsauoussidis and Matta [TM01] outlined major features that transport protocol designers have to work on:

- Correct detection of the nature of error (duration, frequency, etc.)

- Implementation of different error-recovery strategies (aggressive, conservative or more fine-tuned) which are sensitive to the nature of error detected

- Optimization of energy expenditure and connection time utilization

- Bypassing the problems of asymmetric links or having different mechanisms handling problems in each direction

Tsauoussidis and Matta added that transport protocols for mobile ad hoc networks need new performance metrics, other than traditional effective throughput and total expended time, to fairly judge the protocol's performance. They proposed the use of energy efficiency and transmission overhead as more appropriate metrics. These 
metrics address the nature of the battery-powered devices that typically empower wireless and satellite networks.

\subsubsection{Error Control Strategies}

Many studies showed that the ability of a protocol to correctly classify the nature of the detected error can make all the difference for the performance of such a protocol [TB00, TBGP00, BV98, Sam99, KLB99, TBV00, BPSK96, HV99]. Not less important is the ability to take the right action in light of the perceived loss and to predict future losses. Protocols that lack the ability to distinguish errors run the risk of taking an aggressive stance in response to deteriorating link conditions, or wasting bandwidth resources in response to infrequent transient errors. Both situations lead to inefficient energy utilization by the protocol.

In the absence of explicit network feedback, transport protocols need to rely on other methods to distinguish the different error conditions. Congestion errors, common in wired networks, occur when one or more intermediate routers overflow as a result of being overwhelmed by the incoming traffic. These errors are usually accompanied by noticeable increase in delays. All reliable transport protocols should respond to this event by slowing down their transmission rate, or in other words shrinking their sending window. Failure to do so can affect the fair sharing of bandwidth between competing connections. A more catastrophic result can happen when the network reaches congestive collapse, a situation where large numbers of packets 
get dumped from the routers.

On the other hand, link errors can vary in nature. According to Tsaoussidis and Matta [TM01], link errors are usually characterized by both their duration and frequency. Generally, the more frequent the link errors, the worse the throughput gets. As standard transport protocols mistake these errors for congestion, they slow down their transmission and hence become unnecessarily overconservative. Even transport protocols with better error control strategies can expend some transmission overhead investigating the error condition. Determining the exact cause of a link error is quite intriguing. However, it is usually enough to conclude on the burstiness and frequency of such an error.

\subsubsection{State of the Art}

The Transmission Control Protocol (TCP) has become the defacto standard for the Internet today. More than twenty years of research have produced a protocol that is perfectly optimized for today's high performance networks. Naturally, TCP was one of the first chosen transport protocols for the new wireless, satellite, and heterogeneous networks. Quickly, it became obvious that the protocol needs some improvements to perform as well in these new environments. Many researchers took the approach of proposing extensions for TCP to make it more efficient in these networks. Other research efforts went on to verify the achieved results. The availability of current test beds and the strong user base are definite advantages to this approach. However, the 
burden of staying compatible with legacy systems is a major obstacle confining the improvement of TCP.

Other researchers took the approach of developing new transport protocols that are more tailored to the characteristics of their network environment. Unlike the first approach, this approach does not involve the painful process of retrofitting proposed extensions into TCP, but rather involves incorporating a lot of them in the original design of the new protocol. This approach usually adds more integrity and less complication to the protocol. However, it runs the risk of not complying to the standards. This approach also lacks rigorous and comprehensive testing schema, usually defined and available for TCP.

One of those protocols, proposed by Katz and Henderson [HK97, Kat99], is the Satellite Transport Protocol (STP). This protocol is designed exclusively for satellite networks. The authors try to keep interface and feature parity with TCP, while designing their protocol from the ground up with satellite networks in mind. Specifically, they address the problems of asymmetry, variable round trip times and degraded performance in the presence of multiple errors per round trip. These features among others make this protocol better suited than TCP for use in satellite environments. However, the STP protocol is lacking one fundamental feature that is essential for any transport protocol targeted for heterogeneous networks. This missing feature is a discriminating error control mechanism. Unfortunately, STP inherits the assumption 
that any error results from network congestion and is therefore mitigated by applying congestion control measures. This inability to classify and properly handle different error types usually compromises both the protocol's throughput and expended energy.

\subsection{Thesis Contribution}

This thesis proposes a new error control strategy for STP that makes it more adaptive to the unique error conditions in satellite access networks. The new strategy is based on an end-to-end probing mechanism that uses the persistence of the error condition as an indication to the kind of prevailing error in the network. The mechanism works by suspending data transmission when an error is detected and investing some time and transmission effort to sense the current delays in the network. The mechanism associates error conditions accompanied by noticeable delays with network congestion. Otherwise, it associates these errors with different link error events. The strategy proposed here is based on an earlier one proposed by Tsaoussidis and Badr in the context of TCP called TCP-probing [TB00]. However, the new strategy leverages many unique features of STP to enhance the quality of error control. It reuses STP's acknowledgment polling cycle as a probing mechanism and for early error detection. It also uses STP's selective negative acknowledgement as an explicit error indication and a way to detect premature activation. Finally, it makes use of STP's lack of reliance on timeouts to finish faster. The new probing mechanism preserves the endto-end semantics of STP and is implemented as a configurable option in the sender 
only.

Using simulation, this thesis shows that the new probing mechanism for STP indeed improves the effective throughput and increases the energy efficiency of the protocol under various network error conditions. The specific cases studied in this thesis are common in LEO satellite access networks. These cases include three link error conditions: bit corruption; handoff; and limited connectivity, in addition to network congestion. The simulation is divided into categories of tests; each one simulating a network connection that experiences phases of both congestion and one kind of link error. The performance of STP with probing is compared in every test with the one without it. Three performance metrics are measured: the effective throughput, which is the average data reception rate at the receiver; the transmission overhead, which is the percentage of extra bytes transmitted during the reliable transport of data; and the ratio between them. While the first metric is used to determine where a protocol lies in the conservative/aggressive scale, the second one reflects on the level of energy expended in the transmission. This level gains more importance in LEO satellite access networks where various battery-powered devices (LEO satellites and satellite access devices) typically exist. The third metric expresses the protocol's ability to manage the tradeoff between effort expended and throughput achieved. The results show that the new probing mechanism makes STP yield higher levels of throughput, while maintaining lower levels of overhead in most error conditions. 
The whole simulation is carried in PIX (Protocol Implementation Framework for Linux)[Bar02a, BB02, Zha02], developed in the School of Computer Science at Carleton University. The main component of the simulation framework is XSTP eXtended Satellite Transport Protocol. XSTP is a software implementation of the STP protocol in PIX. PIX offers the implementation a preestablished object-oriented design pattern, ease of configuration and deployment and access to light-weight libraries. The new probing mechanism is also implemented as a configurable option in XSTP and is called XSTP-probing. Both the protocol and its probing mechanism are to be included in a new light-weight protocol stack developed specifically for a LEO satellite access network experiment [Bar02b], done by the School of Computer Science at Carleton University.

In addition to XSTP, three other protocols are implemented as part of the PIXbased simulation framework. The first is a simple data streaming protocol called APP. This protocol generates bulk data traffic, which is the traffic pattern used in the tests. The second protocol, called QLP, is a link protocol based on the POSIX message queues to simulate virtual networks on a single physical machine. The final protocol, called XDELDROP, is based on a similar $\mathrm{x}$-Kernel ${ }^{1}$ protocol called VDELDROP [TB00]. The protocol encompasses an algorithm that could be configured to simulate different error conditions in a network by applying different levels of delays and drops

\footnotetext{
${ }^{1} \mathrm{x}$-Kernel is another function-oriented protocol implementation framework [HP91]
} 
to forwarded packets.

\subsection{Thesis Outline}

The rest of the thesis is organized into six chapters. Chapter 2 reviews the literature of the available transport protocols and some proposed extensions for improving them in satellite access networks. Chapter 3 describes the design of the new XSTP protocol in the PIX framework. Chapter 4 details the new XSTP-probing mechanism. Chapter 5 explains the simulation framework including the application, network and error generating protocols. Chapter 6 presents and reflects on the simulation results. Chapter 7 concludes by giving a summary and mentioning issues for future work. 


\section{Chapter 2}

\section{Background and Related Work}

Mobile ad hoc networks, particularly satellite networks, have attracted a lot of attention from the research community since their early introduction. Several propositions have been made to enable data communication over satellite networks. However, one of the main challenges of this field is the efficient transport of data across these networks. Initial studies [AGS99, $\left.\mathrm{ADG}^{+} 00, \mathrm{JBB} 92\right]$ were conducted to evaluate the performance of existing transport protocols. Most of the studies focused on the Transmission Control Protocol (TCP) as a prime candidate, due to its popularity in the Internet. An overview of TCP is given in Appendix A. The collective conclusion of these studies were disappointing as it revealed the suboptimal performance of TCP in mobile ad hoc networks. The most important reason for that lackluster performance is the inherent bias in TCP towards congestion control as a prime form of flow control. Other reasons include some assumptions made by TCP about the characteristics of lower protocols that are more aligned with high-speed wired networks than with 
mobile ad hoc networks. Since these results were published, there has been a number of propositions to enhance the performance of TCP in these networks. Most of these propositions try to boost TCP's performance by suggesting new or modified algorithms without violating the standards published by the Internet Engineering Task Force (IETF). Other studies proposed new protocols that are more targeted towards mobile ad hoc networks in general or satellite networks in particular.

This chapter focuses on reviewing transport protocol propositions, particularly in the context of LEO satellite access networks. It starts by enumerating some of the important extensions to TCP, particularly those that address the problems of LEO satellites. These extensions are classified into three groups: control parameter related, flow control related and error control related. There is a particular focus on error control strategies as they are most relevant to the work in this thesis. After that, some different satellite transport protocols, like STP and SCPS-TP are discussed. There is a particular focus on STP as it provides a context to the contribution in this thesis.

\subsection{TCP Proposed Extensions for Satellites}

The behavior of TCP is described by RFCs and standardized by the IETF. While this behavior is optimized for fast wired networks, it is not equally efficient for mobile ad hoc ones, such as LEO satellite networks. However, there has been a tremendously 
concerted effort by the research community to come up with new extensions to TCP, allowing it to behave more efficiently in these environments. Most of these initiatives are tracked and logged by the IETF [AGS99, $\mathrm{ADG}^{+} 00$, JBB92] in an attempt to promote further experimentation by the research community before adopting any of them as a standard.

\subsubsection{Control Parameter Proposals}

\subsubsection{Initial Window}

The TCP slow start algorithm starts by injecting an initial window number of segments into the network. While it sounds logical to make this window as large as possible to jump start the connection, a standard size of "one" segment is usually recommended. This size is considered a safeguard against transmitting an overwhelming number of segments that can cause more degradation to an already congested network. Alternatively, this segment size can put a damper on the overall throughput of a network with intermediate routers that either have limited size buffers or lower transmission speeds. However, according to Allman et al. [APS99], it is acceptable to increase the initial window to three or four segments at the start of a connection, while keeping it at one segment for further slow start reentry.

One advantage of this increased initial window is to save up to three RTTs from the connection startup time. Another advantage is to decrease the time necessary to trigger the fast retransmit algorithm when a packet is detected lost and the number of 
outstanding segments is small. This second advantage is practically useful in the case of receivers that delay sending ACKs by acknowledging every other segment. The obvious disadvantage of this proposition is the potential risk of causing congestion at the start of a connection. However, one study conducted by Shepard and Partridge [SP98] showed that a slow start with four segments is no worse than another with only two segments after two RTTs. Another simulation study by Poduri and Nichols [PN98] proved that this large initial window extension is useful for short-lived connections in terms of their response time, without excessively increasing congestion losses. In a LEO satellite environment, where link errors are significant, this extension can reduce the time required for error recovery, especially at connection startup time.

\subsubsection{Maximum Segment Size}

The Maximum Segment Size (MSS) of a TCP connection is limited by the network path's Maximum Transfer Unit (MTU). According to Stevens [Ste94], a small MSS is beneficial for interactive applications, which are sensitive to perceived delays. Their small and usually fragmented traffic will not have to wait for large segments to fill before getting transmitted. On the other hand, a large MSS is more efficient in terms of header overhead but can make segments more susceptible to corruption by a bit-error over links characterized by high bit-error rates (BER), such as satellite links.

In addition, the larger the MSS, the more aggressive the session becomes in terms 
of its number of bytes transmitted per unit time (assuming the session does not use burst reduction techniques). This incidence usually increases network congestion, resulting in faster termination of slow start and limiting the future growth of cwnd. Furthermore, the larger the MSS, the longer the session's round trip, affecting loss recovery in two ways: the larger MSS increases the Retransmission Timeout (RTO), which leads to a less timely loss recovery, and it makes it slower for DUPACKs to arrive and trigger the fast retransmit algorithm. Since this size can cause a tremendous effect on the overall performance of a TCP session, extensions that help find an optimal value for it are invaluable.

\subsubsection{Receiver Window}

The size of the receiver window is advertised with every transmitted ACK. This size is set to the free space in the receiver's buffer and is usually used as a receiver-driven congestion flow mechanism. Therefore, the size of this window can affect the performance of the session. In the best case, this size is set to the available capacity in the network, preventing buffer overflow and avoiding over-buffering (excessive queuing resulting from router buffers being too large for link utilization). However, the foregoing is not usually the case since receivers have no knowledge of the network conditions. A small window size can help a connection by reducing the possibility of congestion. However, a too small window size can put a damper on the sender's window growth or disrupt the error recovery by reducing the number of outstanding 
segments. A detailed analysis of the effect of this control parameter on performance is given by Stallings in [Sta00].

\subsubsection{Sender and Receiver Buffers}

In a LEO satellite environment, every resource is very precious and memory is no exception. To accommodate a particular number of simultaneous connections, TCP must allocate enough buffer space for each connection. A recent study by Semke et al. [SMM98] showed that TCP requires at least a bandwidth-delay product worth of buffer space at each end of the connection to operate optimally. However, it is both hard and impractical to compute the bandwidth-delay product since it can span several orders of magnitude across different paths in the network. In the best case, the knowledge of networking experts is required, who are not usually available to manually tune these buffer sizes. The study proposed by Semke et al suggests a new mechanism to adaptively tune buffer sizes based on network conditions and system memory availability. Having such a capability could be priceless when memory resources are in strong demand and short supply.

\subsubsection{Round Trip Time}

Many existing implementations of TCP measure one RTT per window, which scales fine for smaller windows. For larger windows, sampling one segment in a window results in very poor RTT estimates. One way to improve RTT estimation is to use the 
timestamp option [JBB92]. Senders place timestamp values in every outgoing segment and receivers reflect this value back to the senders in the acknowledgments. The senders will then calculate the round trip time by subtracting the echoed timestamp from the current time. Senders and receivers do not have to be clock-synchronized to use this option. The only requirement for this value is to be monotonically increasing. The accurate estimation of RTT is invaluable to many TCP algorithms and extensions. This accurate estimation also produces a more accurate RTO value, which can help prevent bogus retransmissions and achieve a more timely recovery. On the other hand, for a small value of MSS the overhead of sending a timestamp with every segment can be quite high.

\subsubsection{Connection Handshaking}

Some TCP connections are defined as short-lived or transaction-oriented. They are typically found in interactive applications, like web browsers and telnet terminals. For those connections the overhead expended in connection setup and termination is usually not satisfactory. At least three segments are required for connection setup and four more for connection termination. This requirement is above three other segments needed to reliably send the data: one for sending the request, one for sending the reply along with an ACK for the request, and one to finally $A C K$ the reply. One solution, described by Stevens in [Ste96], is called TCP for Transactions (T/TCP). The T/TCP extension mitigates the mentioned overhead by adopting an accelerated 
open strategy.

T/TCP assigns a 32-bit incrementing connection count to newly-opened connections in the form of a TCP option (CC), sent out with every segment. A host maintains a cache of the last $C C$ value received in an acceptable $S Y N$ message from each other host. The transaction sequence starts when a client sends an active open segment to a server containing the client SYN, the request data, the client FIN and client CC option. The server compares this CC value to the one in the cache. If it is greater, the SYN is considered new, the data is passed up to the server, and the connection is called half-synchronized. If no cached CC exists for this host or if it is not greater than the $\mathrm{CC}$ value in the cache, the normal three-way handshake is carried out. After processing the request, the server sends the client a segment containing the server SYN, the reply data, the server FIN, the ACK for the client FIN, the server $\mathrm{CC}$ option and an echo of the client $\mathrm{CC}$ option (CCECHO). This ACK covers all the sequence space up to the FIN. The connection is then called fully-synchronized. Finally, the client sends the server a segment containing the ACK for the server FIN.

$\mathrm{T} / \mathrm{TCP}$ is a very interesting extension due to its minimal set of changes to TCP. Its improved transaction response cycle is also an advantage for real-time constrained applications that might exist in LEO satellite networks. 


\subsubsection{Flow Control Proposals}

\subsubsection{Byte Counting}

The slow start phase is providing TCP senders a safe access to the network. However, Allman et al. $\left[\mathrm{ADG}^{+} 00\right]$ showed that this phase can also waste available network capacity, particularly when coupled with delayed acknowledgements at the receiver. Senders increase their congestion window based on the rate of arriving ACKs, not on the number of acknowledged bytes. When receivers delay their ACKs in the reverse direction, the growth of cwnd slows in the forward direction. Byte Counting, rather than the standard ACK counting, is a sender-based mechanism, proposed by Allman [All98], to mitigate such a problem. Using this mechanism, the congestion window cwnd is increased based on the number of acknowledged bytes covered by the arriving ACK, not the number of those ACKs. This mechanism effectively decouples the congestion window growth from any ACKing interval used by the TCP receivers. Let ackbytes be the number of acknowledged bytes in an arriving ACK, then cund is updated using the following formula:

If $c w n d \leq$ ssthresh then

$$
c w n d=c w n d+\text { ackbytes }
$$

else

$$
\text { cwnd }=\text { cwnd }+\frac{\text { ackbytes }}{\text { cwnd }}
$$




\subsubsection{Transmission Pacing}

The TCP self-clocking property is very useful in getting the connection gradually up to speed based on the experienced network conditions. However, TCP senders using byte counting might get into a situation where they need to send a burst of segments into the network in response to a delayed ACK that is acknowledging multiple segments. This burst of data can cause or proliferate congestion by overwhelming the bottleneck router. In the context of LEO satellites, connectivity can also be temporarily lost and later resumed. As explained by Allman et al. $\left[\mathrm{ADG}^{+} 00\right]$, network conditions could have changed enough that if TCP senders choose to reuse their last cwnd, they can end up being too aggressive by introducing bursts into an already congested network.

Rate-based pacing, introduced by Visweswaraiah et al. [VH97], is a technique to mitigate this problem by introducing a new timer in the sender to pace the transmission of outgoing segments with a specific rate. This timer can be used throughout the connection or get terminated with the first arriving ACK to avoid any significant overhead. The appropriate pacing rate can be either deduced from prior trials or set as a parameter. This technique effectively reduces potential bursts that can overwhelm the network. 


\subsubsection{Disabling Delayed Acknowledgements}

As explained by Stevens in [Ste94], delaying ACKs is a feature used by TCP receivers to attempt to piggyback ACKs with outgoing data segments in the same direction. The most common value for this delay is $200 \mathrm{msec}$. However, if no data segment is transmitted within this period or if another segment arrives, an $\mathrm{ACK}$ is sent right away. Since TCP's self-clocking property depends on the rate of arriving ACKs, this feature can significantly affect performance. One solution can be to turn off this feature, particularly during slow start, to keep the pace of arriving ACKs required for cwnd growth. However, in a connection where segment inter-arrival time is bound by transmission delays, which can be larger than $200 \mathrm{msec}$, this solution does not practically affect performance. This large inter-arrival time is common for wireless and slower LEO satellite links.

\subsubsection{Selective Acknowledgements}

TCP receivers usually use a cumulative acknowledgement strategy to inform senders of the last correctly received segment. However, this strategy fails to inform senders about those segments that are received out of sequence and cached in the receiver buffer. In light of this missing information, TCP senders can best recover at the rate of one segment per RTT. However, if this missing information is available to senders, they can recover from all these losses in much fewer round trips. 
The ability to provide missing information, is the basic rationale for the TCP selective acknowledgement (SACK) option described in [JB88] by Jaconson and Braden. This option contains a list of blocks, describing the sequence space for those segments that are received and cached within the window. Each block is 16-bit containing an origin, relative to the $\mathrm{ACK}$ number field, and a size. The only limiting factor to the number of blocks is the 44 bytes maximum TCP options size. Also, two of those bytes are reserved to inform TCP about the use of this option in the ACK segment. Once the sender receives back an acknowledgement containing a SACK option, it analyzes the sequence of blocks and retransmits only those segments occupying the holes.

Some TCP extensions already make use of this option to take smarter decisions about segment retransmissions. Generally, these TCP extensions try to decouple the choice of what segment to send from when to send it. However, as shown by Hayes in [Hay97], most of these algorithms generate a large line-rate burst of data at the end of loss recovery. Therefore, some sort of burst suppression algorithm is required. In LEO satellites networks where link errors are dominant, this extension can be very helpful in utilizing the available bandwidth.

\subsubsection{Acknowledgement Compression}

According to Allamn et al. $\left[\mathrm{ADG}^{+} 00\right]$, satellite networks can have their reverse links shared by many satellite receivers, as in VSAT networks, or supported by terrestrial modem links. In both cases, there is a high asymmetry between the forward and 
the reverse links. In the case of TCP, its self-clocking property grows the congestion window in the forward direction relative to the acknowledgement arrival rate in the reverse direction. Therefore, a slower reverse link can restrict the throughput of a forward link by hampering the speed of returning ACKs. This asymmetry can be as significant as $75: 1$, resulting in an unacceptable throughput for the TCP connection.

One proposal, by Balakrishnan et al. [BPK97], to mitigate this problem is to have TCP receivers adapting their ACKing behavior based on the network conditions in the reverse direction. One way to communicate a building congestion situation to the receivers is by having routers drop ACKs or mark them with a special congestion pending flag, which can be reflected back by senders in new data transmissions. In response to these notifications, receivers can perform multiplicative backoffs to their ACKing rate. Once the receivers learn that congestion is relieved, usually by getting segments with their flag turned off, they can speed up by applying linear increases to their rate. However, it is always recommended to generate ACKs at least once, but ideally several times per RTT.

\subsubsection{Error Control Proposals}

Reliable transport protocols need to implement error control strategies that adapt to the nature of the detected error. The ability of a protocol to correctly classify and appropriately recover from errors translates into direct gains in throughput and energy saving. On the other hand, poor error control decisions hamper throughput, 
increase energy consumption and subdue performance. The issue of energy saving is particularly of great importance for battery-powered devices like LEO satellites. A study by Tsaoussidis et al. [TBGP00] showed that it is not always wise to tradeoff energy for added gains in throughput. Rather, the balance of these performance goals should be left as a configuration parameter.

Error control is usually two-fold: error detection followed by error correction. Most transport protocols, especially those that were originally designed for wired networks, assume that all segment losses are due to network congestion. This assumption is because these networks have significantly advanced to the degree that link-related errors are almost negligible, which explains why error control strategies in these protocols tend to be congestion centric. While error detection is usually implemented by a retransmission timeout (RTO) mechanism, error correction typically involves reducing the congestion window and backing off the retransmission timer.

On the other hand, different types of errors are dominant in wireless and satellite networks. These errors are either link-related, usually caused by noise or fading channels, or mobility related such as those resulting from hand-offs, signal obstruction and sporadic visibility. Many studies, conducted by Allman, Biaz, Tsoussidis and others [TM01, AGS99, ADG $\left.{ }^{+} 00, \mathrm{KLB} 99, \mathrm{HV} 99\right]$, show that handling these kinds of errors by engaging congestion control can lead to serious performance degradation. Instead, these researchers recommend using recovery strategies that are more tuned 
to the nature of the error. However, the problem of differentiating the error kinds can itself be very tedious, especially in the absence of explicit network feedback and genuine end-to-end mechanisms.

\subsubsection{Proposed Classifications}

There are many classifications of error control strategies for heterogeneous networks in the literature. A recent study by Vaidya [Vai99] gave two classifications of these approaches in the context of TCP. The first classification is based on the nature of the actions needed to improve performance. Generally, these approaches attempt to either hide the link error from the sender or make it more informed about the cause of the error being link-related. The rationale here is to prevent the sender from reducing its congestion window unnecessarily, thinking that congestion is the cause of the error. The second classification is based on where the modifications are needed, which can be at the sender, at the receiver, at intermediate nodes or at a combination of these

places. Furthermore, Vaidya divided the propositions into the following groups: link level, split connection, TCP-aware link, TCP-unaware approximation of TCP-aware link, explicit notification, receiver-based and sender-based approaches.

Ideal transport protocols should take congestion control measures upon experiencing congestion errors, and avoid these measures when the errors are link-related. Alternatively, ideal network behavior should try to hide the link errors from the transport protocols by transparently and efficiently recovering them locally. While it is 
difficult to achieve either one of these two ideals, proposed solutions try to approximate at least one of them. However, for a proposition to be widely accepted, it should not violate the basic semantics of the existing protocols, should inter-operate with legacy systems and should be easily deployable.

Another classification given by Ludwig [Lud99] is shown in Figure 2.1. It divides propositions into five categories depending on the location of the suggested modification. The first category is based on mechanisms that give either the sender, the receiver or both better tools to distinguish the type of error, while retaining the endto-end semantics of their respective transport protocols. These semantics, such as slow-start, congestion avoidance and tolerance to reordered packets are safety factors minimizing the risk of a possible congestive collapse in the network.

A second category is based on the concept of splitting the connection into two at the boundaries of the wired and wireless networks. Proposals in this category rely on the assumption that link errors are more dominant in the wireless part of the connection while congestion errors are more found in the wired part. Therefore, these proposals pre-configure each one of the two connections with the appropriate error control strategy. As detailed by Border et al. $\left[\mathrm{BKG}^{+} 01\right]$, the intermediate node at the boundary of the two networks is called the Performance Enhancing Proxy (PEP). It is important to realize that it is not a requirement that the two connections, separated at PEP, use the same transport protocol. In this case, fake acknowledgements can 
FiII Erad-to- Exd

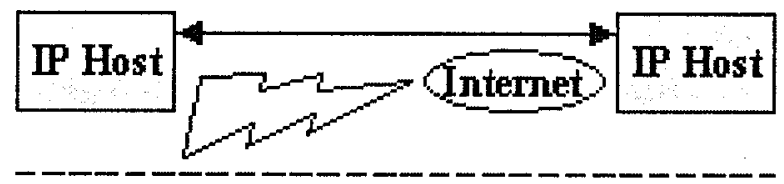

Hard-state Transpart Laye Spiltive

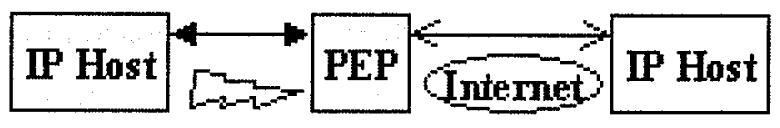

Soft-state Transpout Lyer exthing

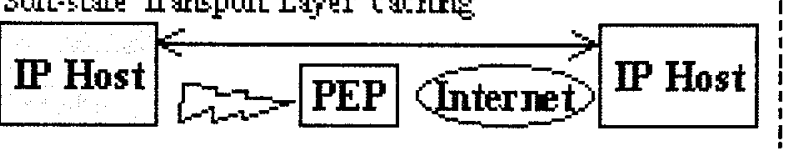

Cross Loprer Sigriline

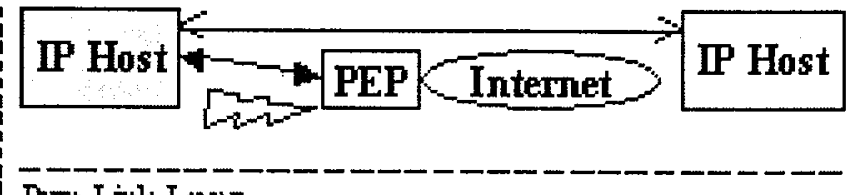

Fure Iimk Las

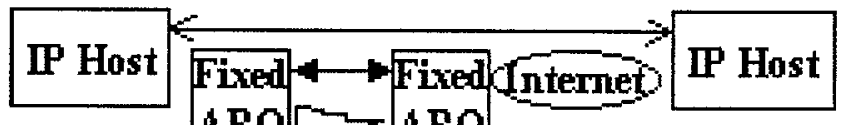

\section{ARO [M- ARO}

Figure 2.1: Error classification techniques for wireless networks [Gur00]

be sent back to the sender at PEP before reaching its destination in the other half of the connection. This practice obviously violates the end-to-end semantics since the overall connection is subject to the interaction of two different connections with two different states. In this case, the PEP proxy is said to maintain a hard state.

The concept of PEP is also utilized in another category of solutions, which depends on maintaining a rather soft state of the connection. Proposals in this category use the same protocol across the connection but maintain a cache of the outstanding packets at PEP. These solutions use the cache to retransmit lost packets in that part of the connection beyond PEP, which is usually more vulnerable to link errors. The Snoop proposal, suggested by Katz et al. [BSK95], is one implementation of these soft-state 
proxy solutions. This implementation recovers link errors locally by retransmitting lost packets from the cache maintained at PEP. This category of solutions has the advantage of preserving the end-to-end semantics of its transport protocols.

A more direct way of classifying an error type is to use explicit notifications, which are typically implemented by means of cross-layer signalling. Error events in other layers are directly communicated to the transport protocol, which handles them by invoking the appropriate error control procedures. Examples in this category include the bad-state [BKPV96] and the explicit loss [BPSK96] notifications. These methods are usually hard to deploy since they require the collaboration of both the terminal and the intermediate nodes of a connection.

The last category consists of pure link layer solutions. As described by Stallings [Sta00], these solutions recover link errors by mechanisms such as Automatic Repeat Requests (ARQ). These mechanisms effectively hide link errors from transport protocols, protecting them from mistaking the errors as congestion-related. However, one disadvantage of these solutions is their possible effect on the round trip time measurement, which is critical to most flow control strategies.

\subsubsection{End-to-End Proposals}

This paper focuses on end-to-end proposals that involve changing either the sender, the receiver or both. These proposals usually introduce heuristics to classify the type of the error. For example, Biaz and Vaidya [BV98] define a class of loss predictors 
based on some congestion avoidance techniques. Biaz and Vaidya's predictors use a simple statistic on the RTT and the throughput to decide on the cause of the error. However, their results are negative as they discover that these predictors are poor at discovering link errors.

In a subsequent work [BV99], Biaz and Vaidya propose a new algorithm to classify the error type by using segment inter-arrival times at the receiver. This approach assumes a network, where only the last hop is wireless and is also the bottleneck link. In this case, the wireless base station typically buffers packets destined for the wireless receiver at the other end of the link and releases them with equal spacing. Meanwhile, the receiver is measuring the inter-arrival time of incoming segments, as illustrated in Figure 2.2(a). When a gap is detected, the receiver gets the size of the gap in segments $n$ and compares it to the noticed increase in inter-arrival time. As shown in Figure 2.2(b), if the increase is equivalent to $n$ times the normal time, the receiver takes this increase as a strong indication of link corruption. Otherwise, the gap is congestion-related. In the latter case and as demonstrated in Figure 2.2(c), out-of-order packets are buffered back to back at the base station, arriving with no variation in the inter-arrival time at the receiver. However, Biaz and Vaidya only confirm their result in the existence of no competing traffic in this last hop.

Another scheme, called the Spike scheme, is suggested by Tobe [TTM $\left.{ }^{+} 00\right]$. It 


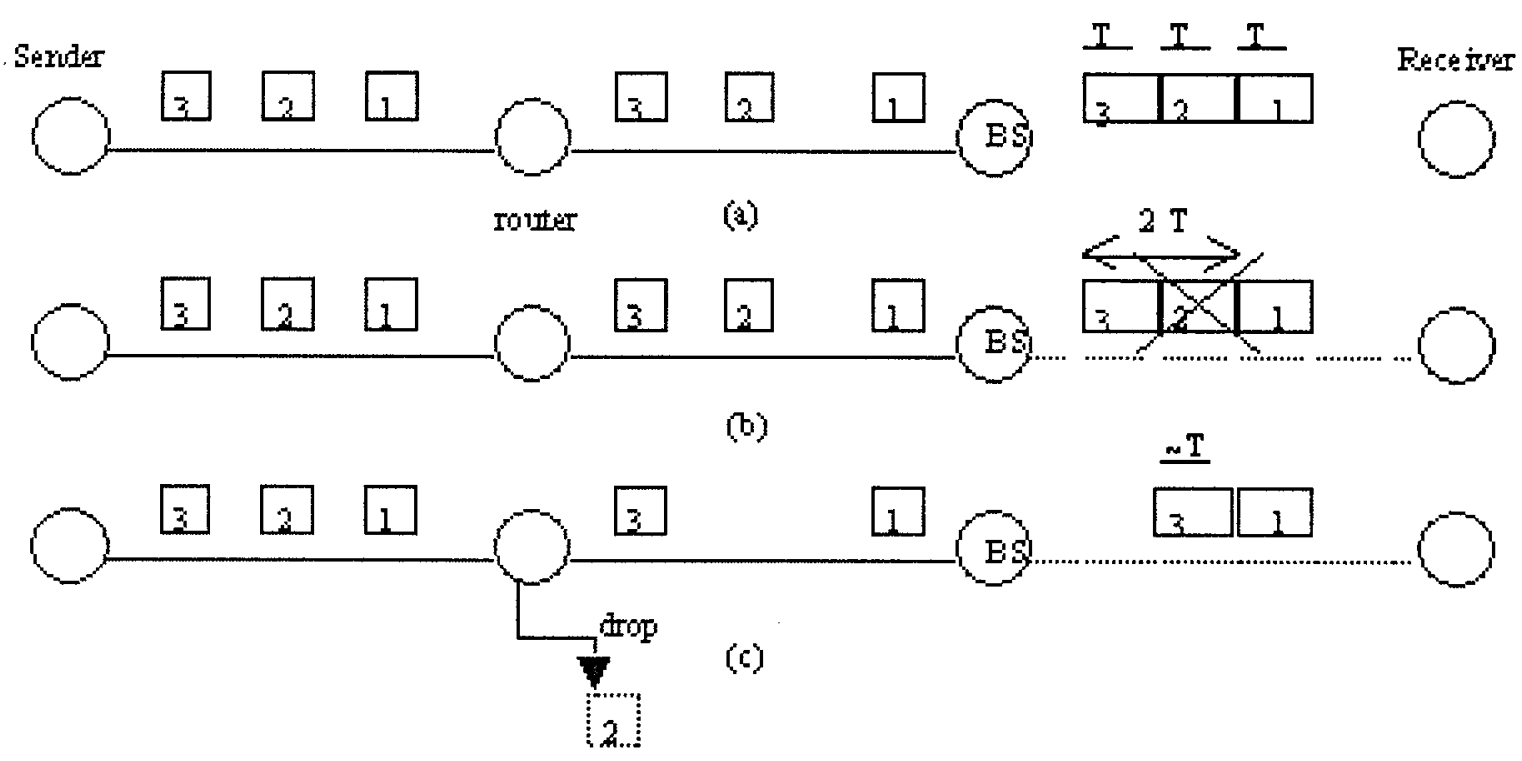

Figure 2.2: Inter-arrival gap scheme [BV99]

utilizes the Relative One-Way Trip Time (ROTT) to identify the state of the connection. ROTT is a measure of the time spent by a packet to travel from a sender to a receiver. ROTT is calculated at the receiver as a relative measure, since the clocks used by the sender and the receiver are usually out of synch. Plotting ROTT versus time produces a graph that shows spikes during the periods of congestion, as shown in Figure 2.3. Therefore, whenever a loss is detected while the connection is in this spike state, the cause is considered to be congestion, otherwise it is link-related. Two thresholds define the start and the end of the spike state and are calculated based on the overall network delays.

A more interesting heuristic proposed by Cen et al. [CCV02] is called ZigZag. It 


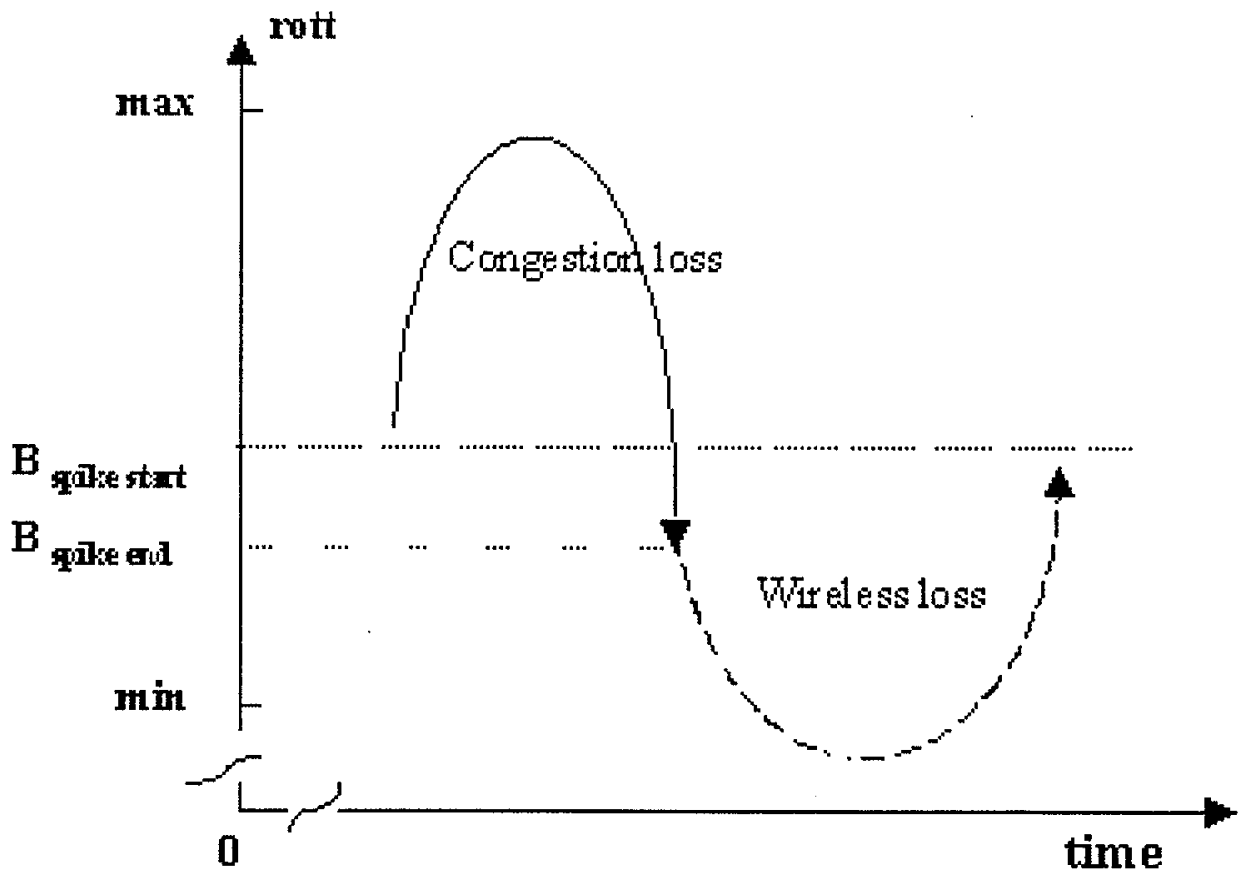

Figure 2.3: Spike scheme [CCV02] 


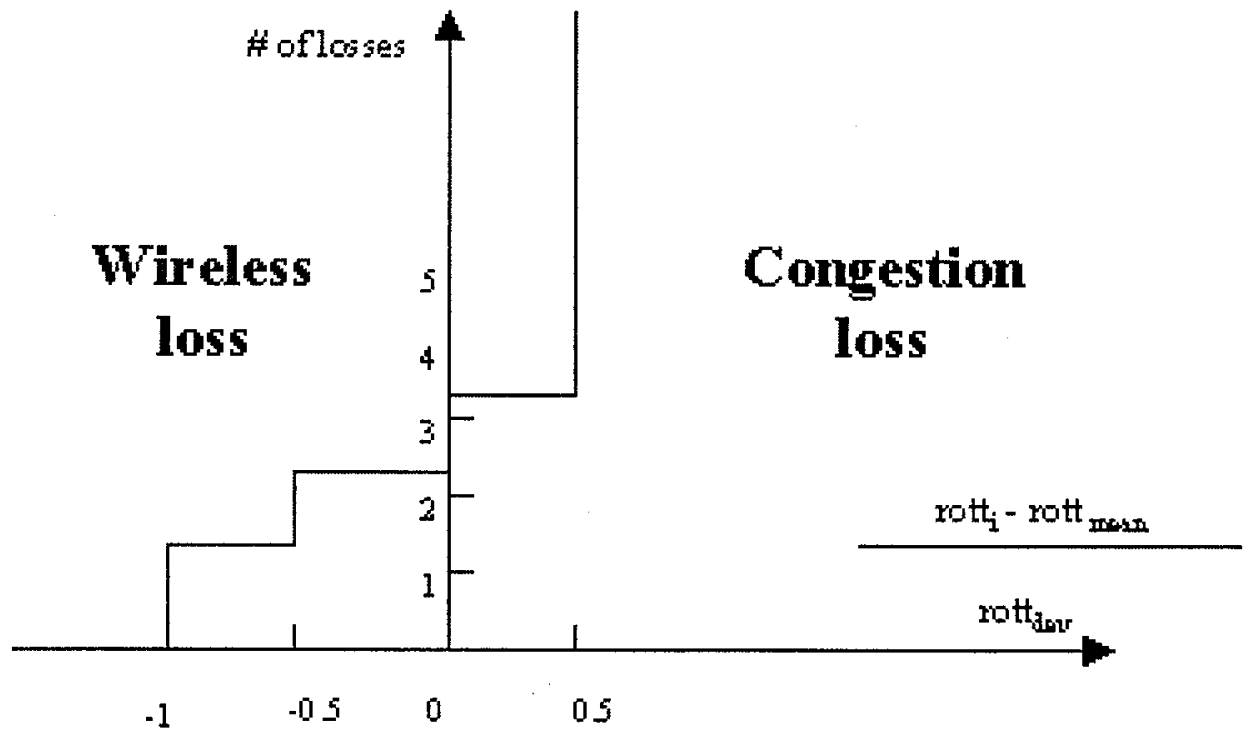

Figure 2.4: Zigzag scheme [CCV02]

performs the error classification based on the number of segment losses $n$ and the deviation of the ROTT measurement at the time of the detection. ZigZag works on the assumption that a more severe loss is usually associated with congestion, which is usually accompanied by higher ROTT measurements than normal. The algorithm outlines certain expected ROTT deviation levels for each amount of loss detected. These levels are proportional to the number of losses. If the deviation noticed exceeded the corresponding level, it is a strong indication that the loss is congestion-related. If that is not the case, it indicates that the noticed delays are normal, usually characteristic of link errors. The scheme is depicted in Figure 2.4. 
Other heuristics need the collaboration of the sender and receiver sides of a connéction. For example, Kim et al. [KLB99] proposed an algorithm called Linear Increase/Multiplicative Decrease with History (LIMD/H). This algorithm uses the history of packet losses and the evolution of the transmission rate to make the loss type classification. LIMD/H algorithm tries to assess whether a sender is sending at, above or below its fair share of the bandwidth. Based on that assessment, it classifies the error into one of three groups. The first group is for congestion errors, which result from transmitting at a rate that is larger than the session's fair value. The second group is for probe errors, which result from performing linear increases in the sending rate to probe the network for additional capacity. The final group is random errors, which basically result from link errors. The effective transmission rate is measured by the receiver and reflected back to the sender in each round trip. Based on the error type, the algorithm suggests taking an appropriate error control measure.

The Wave IE Wait protocol, proposed by Tsaoussidis et al. [TBV00], is another example of the collaboration between the sender and the receiver. The sender in this protocol groups fixed-size segments into waves, whose lengths are dictated by the receiver. Segments belonging to a single wave are transmitted back-to-back by the sender. Using this knowledge about the wave level, the receiver can estimate the total time it should take the whole wave to arrive once the first segment is received. The receiver then uses that information to calculate the effective throughput and 
compare it to a baseline throughput, which is calculated when no congestion exists. If losses are detected with noticeable degradation in throughput, a congestion situation is detected; otherwise, the error is probably link-related. Based on the outcome of this test, the wave level is maintained, lowered or increased. The new level is communicated back to the sender in the acknowledgement, at least once per round trip. The same wave mechanism is implemented later by Tsaoussidis and Zhang in the TCP-Real protocol [ZT01].

Another outstanding work by Tsaoussidis and Badr [TB00] introduced the concept of probing to transport protocols. Upon detecting an error, the sender suspends transmission and initiates a probing cycle, consisting of multiple exchanges of small segments and their acknowledgments between the sender and the receiver. The sender initiates the cycle by sending a probe segment and waits for its acknowledgement. Once received, it sends the next probe and again waits for its acknowledgement. Any missing probe or acknowledgement re-initiates the cycle. For each probe exchange the RTT is measured and at the end of the cycle the vector of measured RTTs is compared with the RTT estimate at the time of detecting the error. If all of those RTTs are less than or equal to the RTT estimate, it is assumed that the error was transient and therefore transmission is restored at the previous levels (Immediate Recovery). Otherwise, congestion is detected and normal congestion control measures 
like halving the congestion window and backing off the RTO are taken. It is interesting to realize that the time expended in the probing cycle is more than made up for if the error turned up to be link-related. The sender, in this case, avoids taking unnecessary congestion control measures, which usually leave the session too conservative. Also, the rationale behind holding off transmission while probing is to save energy that might be wasted in useless retransmissions when the network is experiencing deteriorated conditions.

\subsection{STP: Satellite Transport Protocol}

The Satellite Transport Protocol (STP), proposed by Katz and Henderson [Kat99, HK97], is a transport protocol that is specifically optimized for asymmetric satellite access networks. The protocol provides a reliable connection-oriented byte streaming service to application protocols through a similar interface to TCP. While STP incorporates many of the basic principles found in TCP, it is only functionally but not semantically equivalent to it. The protocol is also modelled after an ATM-based link protocol known as SSCOP. Many of the design principles of SSCOP, originally developed for broadband wide-area ATM networks, are well suited for satellite networks. STP is found to outperform TCP in environments characterized by high BER, severe asymmetry and varying RTTs, typically characteristic of LEO satellite links.

STP sessions can transmit variable length data segments, as shown in Figure 2.5. 
To minimize the control overhead in smaller data segments, STP enforces separation between data and control information. It achieves this separation by defining eight distinct segment types. The first two are the BGN and the BGNAK segments, which are used to establish the connection. The next two segments are the END and the ENDAK, which are used to terminate the connection. The fifth type is the variable length sequenced data segment $(\mathrm{SD})$ with a 24 -bit sequence number and a checksum. SD Segments rather than data bytes are numbered in STP, resulting in a high modulus. These segments are cached before being transmitted in the network, along with timestamps indicating their last time of retransmission. These timestamps are used as a protection against spurious retransmissions. Once these segments get acknowledged, they are removed from the cache.

While TCP's acknowledgement strategy results in a significant amount of ACK traffic in the reverse direction, STP has a more efficient mechanism. TCP receivers acknowledge arriving segments by either sending an ACK for every segment or two (depending on the use of delayed ACKs) or by using the SACK extension. On the other hand, STP employs an automatic repeat request (ARQ) mechanism that uses selective negative acknowledgements (NACK). Only segments reported missing by receivers are retransmitted. Unlike TCP, there is no RTO mechanism in STP, which makes it more resilient to RTT variations.

STP uses two more segments in the ARQ mechanism, namely the POLL and the 


\begin{tabular}{|c|l|}
\hline source port & dest port \\
\hline Woe & $\begin{array}{l}\text { sequence } \\
\text { number }\end{array}$ \\
\hline checksum & instance \\
\hline Oata \\
\hline
\end{tabular}

Sequenced Data 80 )

\begin{tabular}{|l|l|}
\hline source port & degt. port \\
\hline tupe & $\begin{array}{l}\text { sequence } \\
\text { number }\end{array}$ \\
\hline checksurn & instinnos \\
\hline list element 1 \\
\hline list element2 \\
\hline
\end{tabular}

Unsolicited STAT (USTAT)

\begin{tabular}{|l|l|}
\hline source port & dest port \\
\hline type & $\begin{array}{l}\text { sequence } \\
\text { number }\end{array}$ \\
\hline checksum & instance \\
\hline resered & optons \\
\hline \multicolumn{2}{|c|}{ timestamp } \\
\hline
\end{tabular}

FOLL

\begin{tabular}{|l|l|}
\hline source port & dest. port \\
\hline type & $\begin{array}{l}\text { sequence } \\
\text { number }\end{array}$ \\
\hline checksum & instance \\
\hline vindow & options \\
\hline \multicolumn{2}{|c|}{ timestamp } \\
\hline
\end{tabular}

BGN,BGNAK

\begin{tabular}{|c|c|}
\hline source port & des. poit \\
\hline thpe & $\begin{array}{l}\text { sequence } \\
\text { number }\end{array}$ \\
\hline checksum & instance \\
\hline window & options \\
\hline imestamp \\
\hline Istelement 1 \\
\hline Mt element2 \\
\hline
\end{tabular}

$$
\text { STAT }
$$

\begin{tabular}{|l|l|}
\hline source port & desi.port \\
\hline type & $\begin{array}{l}\text { sequence } \\
\text { number }\end{array}$ \\
\hline checksum & instance \\
\hline
\end{tabular}

END, EWDAK

Figure 2.5: STP segment kinds [Kat99] 
STAT segments. An STP session periodically transmits a POLL segment containing a timestamp and the sequence number of the next segment to be sent. An STP receiver responds to a POLL segment by transmitting a STAT segment. This segment contains an echo of the POLL's timestamp, the sequence number of the next expected segment and a gap report. This report lists all the segment gaps found in the receiver's cache of out-of-sequence segments. The STAT segment can be contrasted to TCP's SACK option where only the most recent three gaps are reported. The last segment type in STP is the called the unsolicited STAT (USTAT). This segment is sent by a receiver to quickly notify a sender of a new segment gap, without waiting for the sender's next POLL. A USTAT segment contains the sequence number of the next expected segment in addition to the most recently found gap. However, in a network where packet reordering is a possibility, receivers can delay sending USTATs until there is a high probability of segment loss (usually indicated by receiving $n$ more segments after a gap is first detected). It is important to notice that USTATs are the primary form of NACKs since they allow for a more timely recovery. POLL/STATS cycles, on the other hand, are used to recover all second-order losses. Therefore, POLL/STATS cycles can be scheduled at a lower rate (two or three times per RTT is typical). Generally, this ARQ mechanism results in lower reverse link traffic when the loss is negligible and a speedy recovery when the loss is severe.

STP keeps a smoothed estimate of the RTT based on a timestamp exchange in 
each POLL/STAT pair. When gap reports are received by senders, the reports are inspected on a segment by segment basis. For segments reportedly missing by a USTAT, it has to be their first retransmission. For those segments reported missing by a STAT, one RTT must have passed since a segment was last retransmitted. A segment also must have been last retransmitted before the STAT's timestamp. These validations performed by a sender reduces the chances of spurious retransmissions.

TCP has a self-clocking property (the arrival of an ACK triggers the departure of a new segment) that helps smooth out data transmission at a rate sustainable by the network. However, STP uses another mechanism that adapts to the amount of rate control required in the network, ranging from no rate control to explicit rate control. In the former case, a congestion-centric fow control mechanism that is windowbased, is used. However, unlike TCP, the slow start phase is not reentered (unless transmission is suspended for a very long time) as there are no coarse retransmission timeouts used. In the latter case, an explicit rate flow control is used where the transmission rate is guaranteed not to exceed a certain configurable rate.

Since STP lacks the self--clocking property, it depends on a delayed send timer to pace transmissions uniformly over the estimated RTT. Using that timer, the implementation of the explicit rate control mechanism becomes straight forward. The procedure is performed by putting an upper limit on the timeout of the delayed send timer. The granularity of this timer is usually dependant on the access speed of the 
network interface, and on enforced rates by the network. It is interesting to realize that this pacing mechanism reduces the risk of introducing large bursts to the network, which usually results from the ARQ process.

STP also supports segment type overloading. Certain type combinations are allowed to exist in the same segment. The protocol uses this feature to support a fast connection start mechanism similar to the one proposed in T/TCP [Ste96]. In this case, the data is allowed to be transmitted along with the BGN segment in anticipation of a connection acceptance by the targeted host. This process is done by overloading the segment's type to enable both the BGN and the SD flags. Both the POLL and the END flags can also be enabled, allowing a whole transaction to be embedded into a single STP segment. Furthermore, POLL segments can also be piggybacked with the SD segments when both segments are scheduled to be transmitted at the same time, effectively reducing the total number of segments transmitted to the network.

Finally, it is important to realize that since STP is not semantically compatible with TCP, it cannot directly be deployed to the Internet. However, STP can be deployed either to a private satellite network or to the satellite portion of a TCP connection, which is split at the boundaries of the wired and satellite networks. 


\subsection{SCPS-TP: SCPS Transport Protocol}

The SCPS transport protocol (SCPS-TP) [SCP98] is an experimental protocol specifically designed to address the needs of various transport missions in space. The inception of this protocol comes from TCP and UDP. However, many of the high performance extensions [JBB92] proposed by the research community are incorporated as well. One of the protocol objectives is to support full, partial and minimal transport reliability modes. This protocol is also meant to operate efficiently under a wide range of delays, bandwidth, and error conditions. In addition, the SCPS-TP protocol supports connectionless multitasking and priority-based processing. Above that, the protocol offers several customization options to meet the unique requirements of space missions.

SCPS-TP introduces some new mechanisms to enhance the transport performance in space. The selective negative acknowledgement capability was first introduced by this protocol to allow receivers to send more feedback about the error condition back to the senders on a regular acknowledgement. The protocol also incorporates an explicit error notification mechanism that makes it capable of choosing the most appropriate recovery strategy. Specifically, the protocol can respond to explicit congestion notification, explicit corruption notification and link outage notification. In the former case, the protocol halves its congestion window and doubles its RTT, while in the second case it does neither of these two actions. For the third case, the protocol 
puts its normal transmission on hold and goes into persist mode until the link is back up. These explicit notifications are implemented using an ICAP like protocol. Other extensions implemented in SCPS-TP include header compression, the configuration of the primary source of data loss, and a partially reliable service option.

\subsection{Summary}

Several proposals are made by the research community to mitigate problems characterizing data transport in satellite networks. Most of these proposals are made in the context of TCP. Other researchers took the approach of implementing custom transport protocols that are more tuned to satellite networks. One of the most interesting research topics in this area is the error control strategies employed by transport protocol in satellite networks. A successful strategy is basically able to detect and properly adapt the protocol's transmission rate to the prevailing error condition in the network. It also manages a tradeoff between the achieved throughput and the level of overhead expended to achieve the result. However, an ideal strategy that perfectly manages this tradeoff is yet to be perfected. 


\section{Chapter 3}

\section{XSTP: Extended Satellite Transport Protocol}

XSTP is a software implementation of the STP protocol in the PIX (Protocol Implementation Framework for Linux) framework [Bar02a, BB02, Zha02]. PIX offers a protocol implementation a preestablished object-oriented design pattern, ease of configuration and deployment and access to light-weight libraries. XSTP is the main component of the PIX-based simulation framework that is used in this thesis. The protocol is used to host a new error control strategy, called XSTP-probing, which is introduced in the next chapter. The protocol is also to be deployed as a transport protocol in a new PIX-based communication protocol stack for an experimental LEO satellite access network [Bar02b]. This chapter explains the general architecture and the detailed design of the new XSTP protocol. 


\subsection{General Architecture}

The architecture of XSTP is directly affected by design patterns existing in PIX. The PIX framework clearly separates the concept of a protocol from that of a session. A protocol abstraction defines the basic semantics of the represented protocol like its addressing scheme and capabilities. It also defines the rules of managing the protocol's sessions and performs the actual managing of these sessions. A session abstraction, on the other hand, represents a connection instance. It defines the runtime state of a specific connection and sets the rules of maintaining this state. A typical communication suite defined in PIX consists of several protocols grouped into levels and arranged into a hierarchy. A single connection consists of a chain of sessions; each session belongs to exactly one protocol on each level. PIX declares both the protocol and session entities as passive classes. Rather than having their own threads of control to handle incoming and outgoing messages, these threads are assigned directly to the messages. Messages are data abstractions that are either sent or received. They are created at some level and then passed either up or down the session chain. This process effectively creates a multi-threaded environment handled differently by each protocol. A message is usually interpreted by each protocol as a combination of header and payload. A payload for one protocol typically contains the lower protocol's header and payload combined. An example of a PIX message is shown in Figure 3.1. 


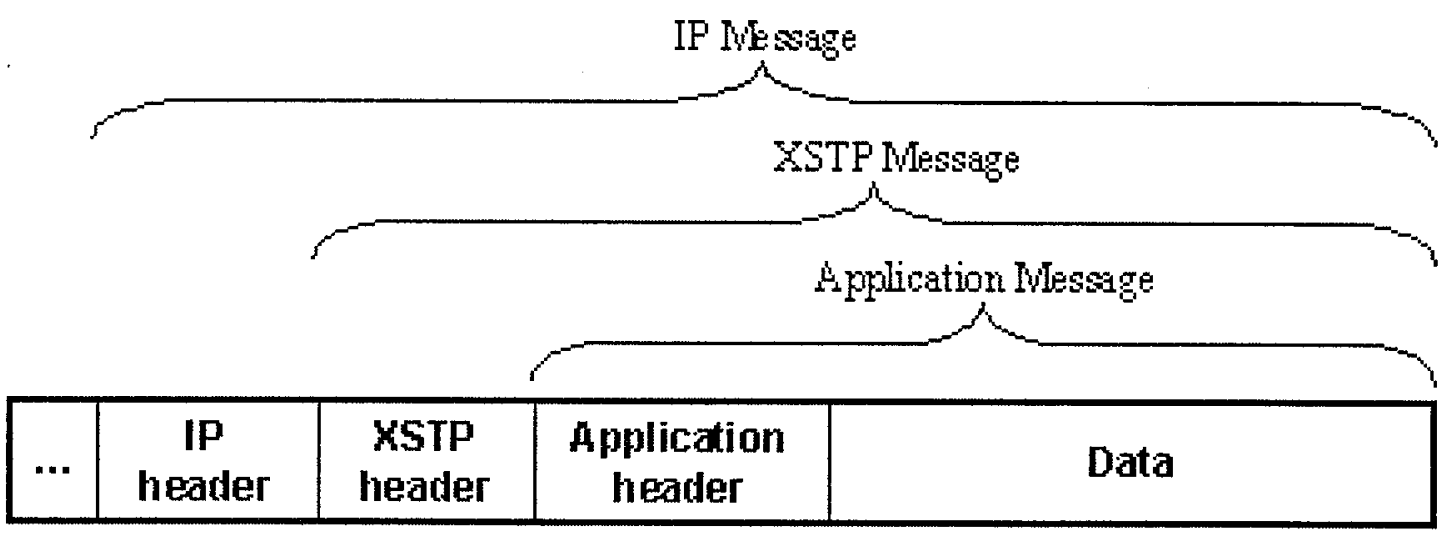

Figure 3.1: A PIX message

In the case of XSTP, the protocol can typically be deployed on top of a network protocol such as the Internet Protocol (IP). XSTP will also provide a reliable byte streaming service to application level protocols such as the File Transfer Protocol (FTP). Furthermore, unlike other sessions in PIX, an XSTP session has exactly one lower and one upper sessions. A typical configuration for a communication suite that includes XSTP is shown in Figure 3.2. When such a suite is initialized, an instance of the XSTP protocol is created, configured and then installed in the appropriate location in the protocol hierarchy. Once there, application level protocols can use the service of the protocol to manipulate XSTP sessions.

As we said, the design of the XSTP protocol conforms to the PIX framework's specifications. Therefore, separate protocol and session entities (classes) are defined. The relationship between them is such that a protocol instance can own one or more 

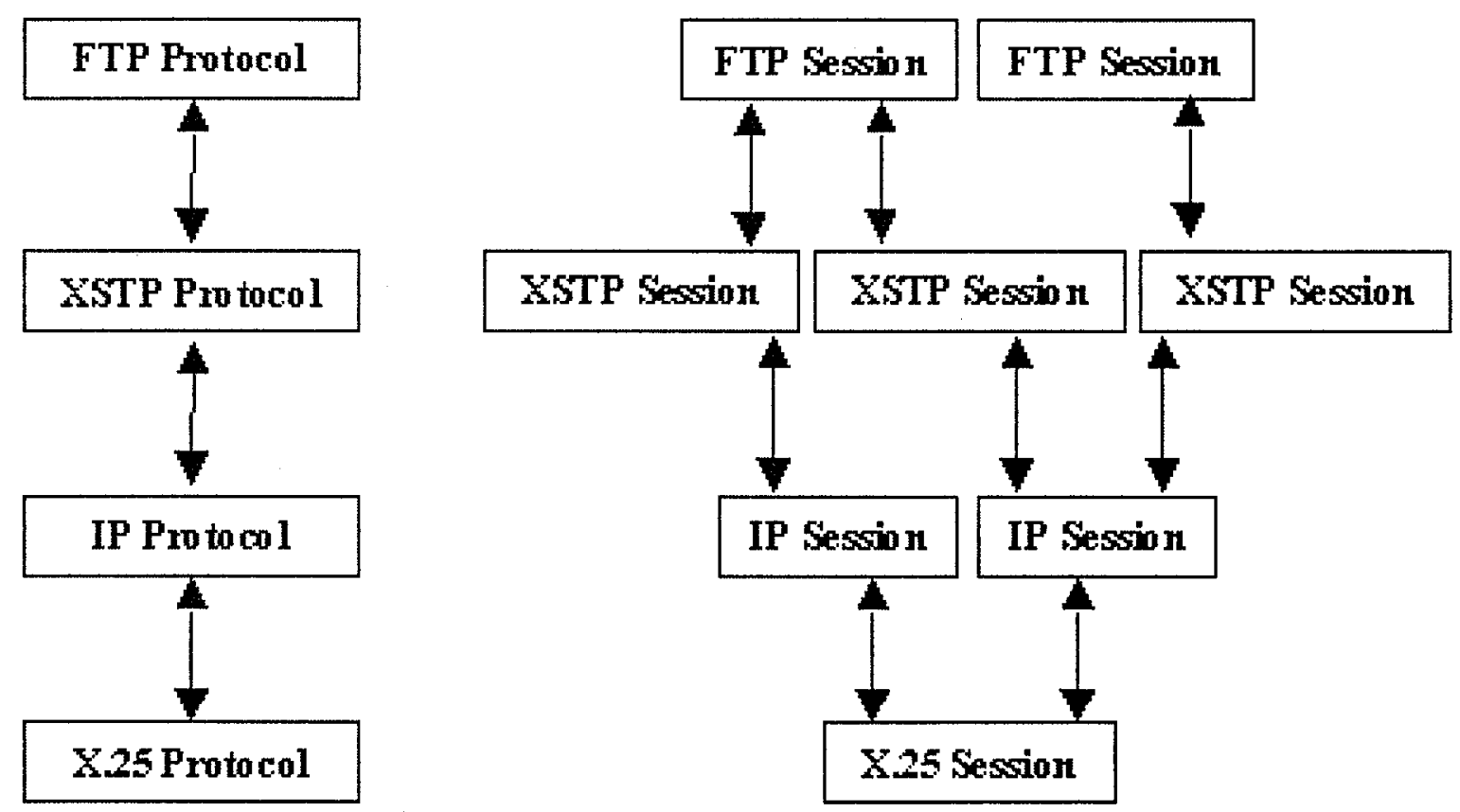

Figure 3.2: PIX protocol and session configurations including XSTP 


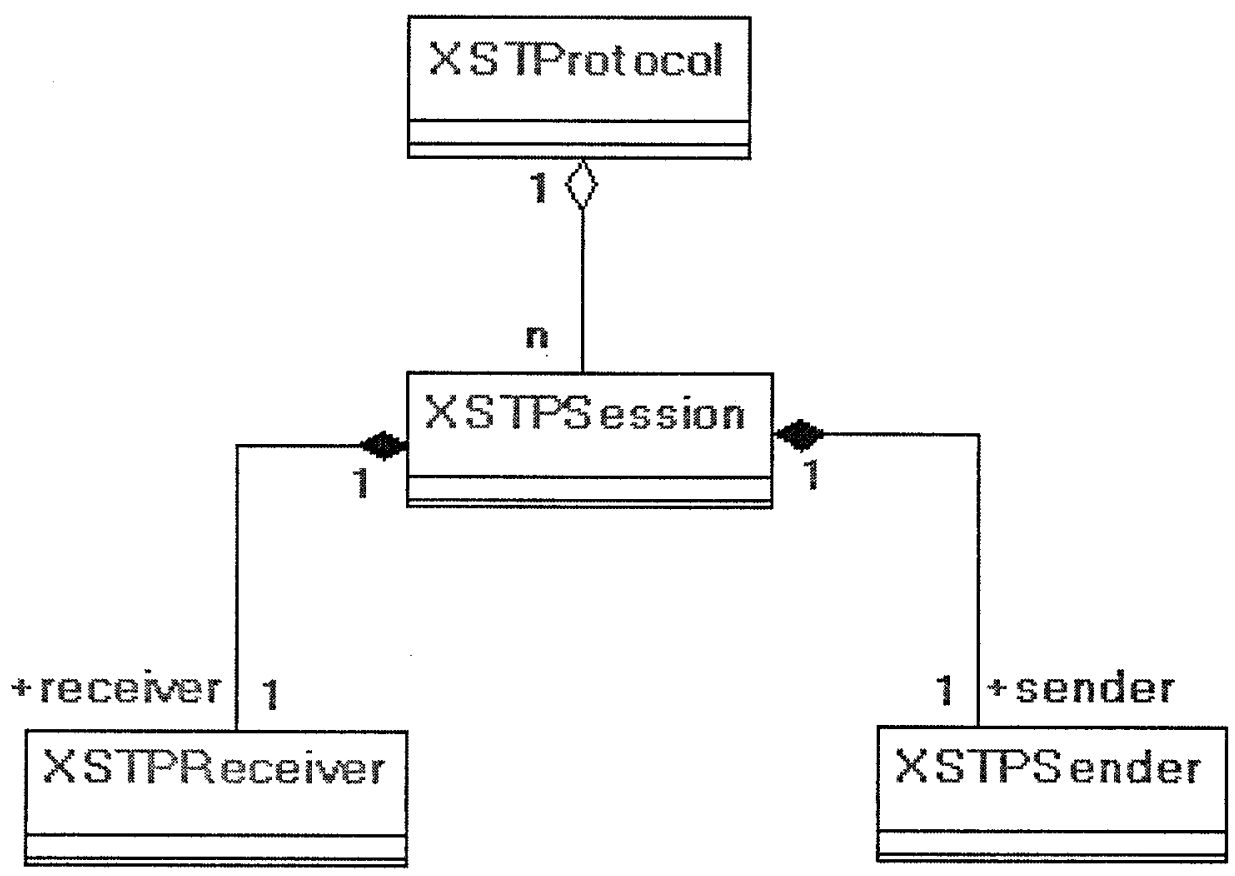

Figure 3.3: XSTP class diagram

session instances. An XSTP session basically plays the roles of both a sender and a receiver. For that reason some of the session's responsibilities are re-factored into two new classes: an XSTP sender and an XSTP receiver. An instance of each of those two classes is created in the private state of the session's object. These two instances play the sending and receiving roles of the session. The complete design is shown in Figure 3.3 and detailed in the following Section. 


\begin{tabular}{|l|}
\hline XSTPPassiveld \\
\hline othspot: Fort \\
\hline
\end{tabular}

\begin{tabular}{|c|}
\hline XSTPActiveld \\
\hline Ssh scot: Pot \\
Sht dort: Port \\
\hline shlls: Sescorr \\
\hline
\end{tabular}

Figure 3.4: XSTP active and passive keys

\subsection{Detailed Design}

\subsubsection{Protocol Class}

The protocol class is called XSTProtocol. Using the framework, it is designed to support both active and passive sessions. While active sessions are created by clients, passive sessions are created by servers. The protocol keeps separate collections of these kinds of sessions. Every session, active or passive, is identified by a unique key. A passive key consists of a listening port, which is a short integer identifying a server's transport address. An active key, on the other hand, consists of a local port, a remote port, and a reference to a lower session. While a remote port is basically a listening port, a local port signifies a client's transport address. However, the combination of local and remote ports is only unique in the context of a lower session, which is the reason for having a reference to that session in the active key. The definitions of both keys are shown in Figure 3.4.

Passive sessions are defined by enable objects. An enable object contains the 
session's passive key and a reference count. An application's server session can register a' listening XSTP port by calling the protocol's $x$ OpenEnable method with a passive key containing the port. This method looks up an enable object for that port in the passive map. If one is found, its reference count is incremented; otherwise a new enable object is created and inserted in the passive map. The advertised listening port is used to accept active session creation requests made by clients of the server session. When the server session needs to stop advertising its listening port, it calls the protocol's $x$ OpenDisable method with the same passive key. This method finds the matching enable object in the passive map and decreases its reference count. If the count becomes zero, the enable object is completely removed from the map. The protocol's $x$ OpenDisableAll is a convenient method to disable all the listening ports of the protocol by emptying the protocol's passive map.

On the other hand, an application's client session uses XSTP to connect to a server's advertised listening port. The client session accomplishes that by calling the protocol's xOpen method with an active key. A client session can either specify a real free port or an ephemeral one (replaceable by XSTP with a real free port) as the session's local port in an active key. However, the key's remote port has to be an existing listening port. The method starts by creating an XSTP session instance, then calling its $x$ Connect method to establish the connection synchronously. If the connection is successfully established, the method returns the session to the client; 
otherwise the session instance is deleted and a null object is returned. The connection use-case is demonstrated in the sequence diagram in Figure 3.5. The client uses the created active session to send and receive data across the connection. When it is done with the session, the client calls the protocol's $x$ Close method to close the session synchronously. Since an XSTP session has only one upper session, the $x$ Close method directly removes the session from the active map, deletes its instance and propagates the close request to its lower session. The disconnection use-case is demonstrated in the sequence diagram in Figure 3.6. Furthermore, XSTP also supports close requests coming from a lower session. Basically, a lower session informs XSTP of its closure by calling its $x$ CloseDone method. The protocol can then close all of its sessions that reference the lower one.

When an XSTP session sends a connection request to a listening port, the received request message is popped from a lower session to the XSTP protocol instance through its $x$ Demux method. The protocol then constructs an active key out of the message's header and uses that key to lookup a session in the active map. For a new connection request, an active session is typically not found. The protocol then constructs a passive key from the same message header and uses it to search the passive map. If an advertised listening port is found, the protocol creates a new active session in the listening state and forwards the request message to this session through its $x P o p$ method. If no matching listening port is found, the request is simply ignored and a 


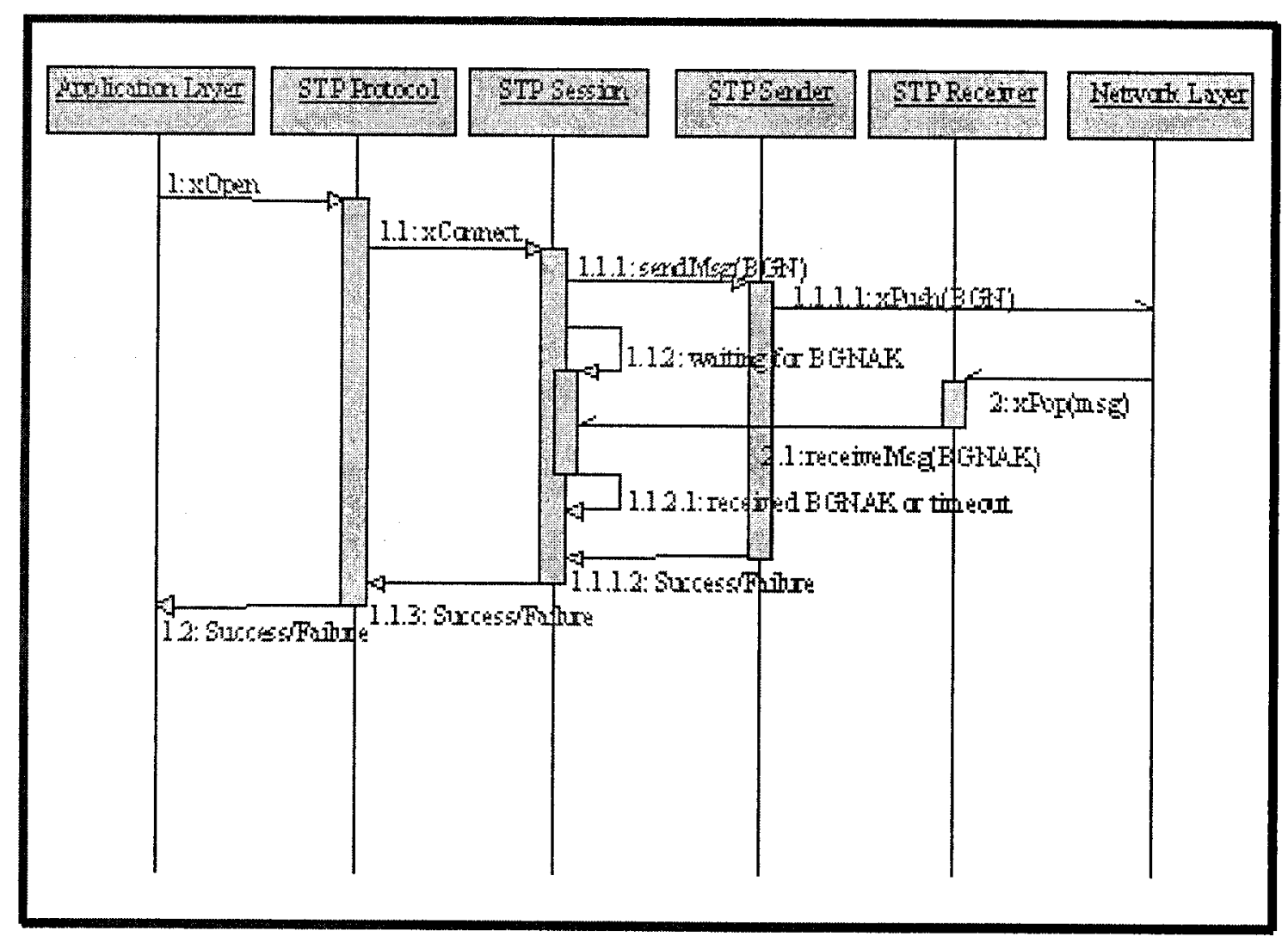

Figure 3.5: XSTP session connection sequence diagram 


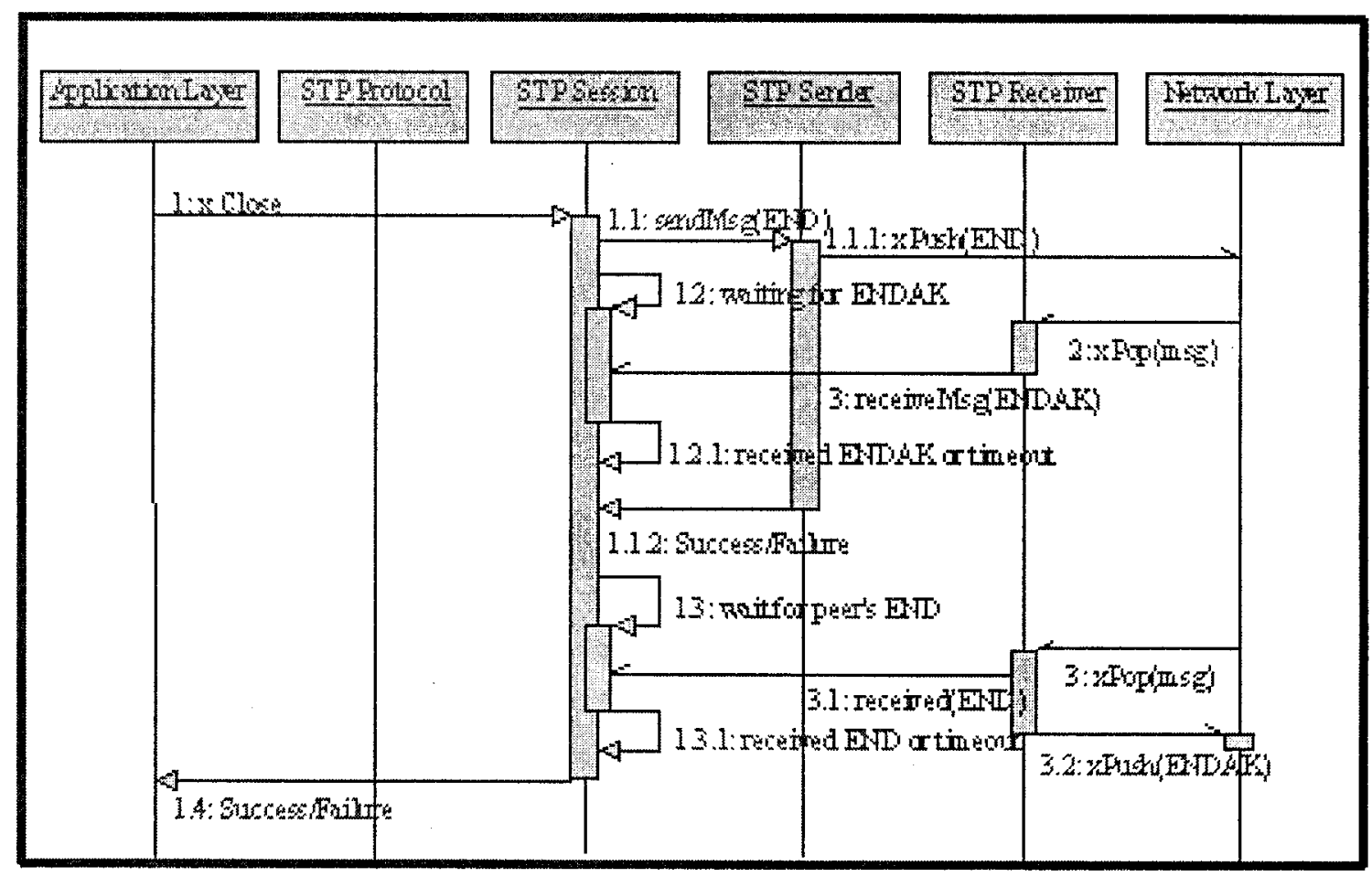

Figure 3.6: XSTP session disconnection sequence diagram 
reset message is sent to the source session.

In addition to its session manipulation capabilities, XSTP also multiplexes arriving messages to their designated sessions. When an incoming message is passed up from a lower session by calling the protocol's $x$ Demux method, XSTP starts by checking the message's checksum. If the checksum test failed, the message is ignored; otherwise the protocol continues its processing. It uses the message's header to create an active key to look up a session in the active map. If one is found, the protocol next verifies that the message belongs to that incarnation of the session (the incarnation number is part of the message header). If it does, the message is popped to the session by calling its $x$ Pop method; otherwise, the message is ignored. If no matching active session is found, the message is also ignored unless it is a new connection request message. In that case, the protocol deals with it as described previously. In the special case where the message is a connection request for a different incarnation of an existing active session, XSTP considers this message an evidence that the peer of that session has closed. Therefore, XSTP also closes the session.

\subsubsection{Session Class}

The session class is called XSTPSession. The definition of this class follows the pattern introduced by the PIX framework. This class exposes an $x P$ ush method to allow client sessions to push data messages down an XSTP connection. The class also exposes an $x P o p$ method to allow its protocol to pop received messages from the other end of the 
connection. As mentioned, the sending and receiving logic is so complex that it has to be re-factored in two other classes: a sender and a receiver. Therefore, the session's implementation of the previous two methods simply delegates to these two helper objects. In addition, the XSTP session exposes an $x$ Control method to allow other objects to communicate with it by getting and setting certain advertised properties. The foregoing is a realization of the PIX inter-layer communication capabilities.

XSTP session instance has a state. In a multi-threaded environment such as PIX, access to an instance's state has to be synchronized, which is the reason to enforce a mutually exclusive access to all the session's entry points. This protection is obviously extended to the session's sender and receiver classes as well. Only one thread is allowed to operate on a session's state at a time. However, multiple threads can still operate on different sessions concurrently. The session typically schedules threads using a first-in-first-out (FIFO) policy.

Each XSTP connection consists of two sessions created on the client and the server sides. An XSTP session is best modelled as a simple state machine like the one in Figure 3.7. While a client session is created in the CLOSED state, a server session is created in the LISTEN state. After being created, a client session starts a handshaking cycle that involves sending a BGN segment (connection request) to the server. It is only after a BGN_ ACK segment is received by the client that the connection handshaking is completed successfully. The two sessions then move to the 
EST state. Similarly in the disconnection process, one of the sessions has to do an active close. This disconnection process is accomplished by starting a handshaking cycle that involves sending an END segment (a disconnection request). It is only after an END_ ACK segment is received by the initiating session that the connection is labelled half closed. For the connection to be fully closed, the other session has to also complete a similar disconnection handshaking cycle successfully. After that is accomplished, both sessions go back to the CLOSED state before having their instances destroyed.

As mentioned, session creation and destruction are synchronous operations; which means that the caller has to wait for these operations to either succeed or fail before moving on. To preserve the integrity of the caller, both of these operations have a timeout mechanism that is triggered only after exhausting several attempts. While the connection handshaking is attempted right after a session is created, the disconnection handshaking is only attempted when the session is in the idle state. A session is in the idle state when it is neither sending nor receiving. In that state, the session typically maintains a keep-alive timer to periodically check (by sending a POLL segment) whether its peer session is still alive.

The XSTPSession class instance maintains the session's state that is common to both the sender and the receiver, such as the session's smoothed round trip time and variance estimates. The XSTPSession class also offers its two helper classes methods 


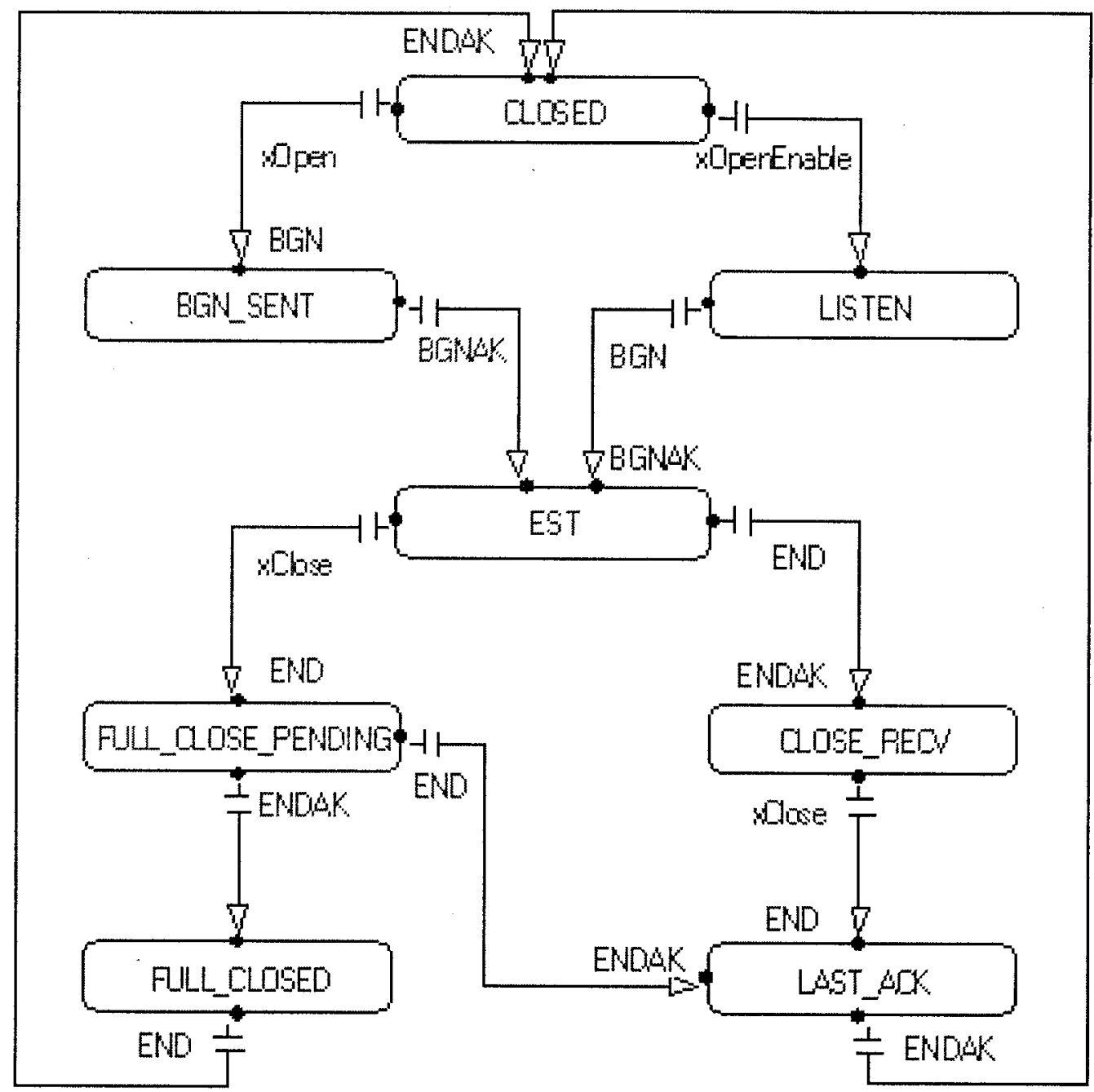

Figure 3.7: XSTP session state machine 
to communicate with other upper and lower abstractions. One of these methods is to notify the upper protocol of a peer session's closure event. Another method is to present received data messages to the upper protocol. A third method is to drop the session and reset the peer in case of error. As noticed, XSTPSession class is just a coordinator of both the sender and receiver classes.

\subsubsection{Receiver Class}

The receiver class is called XSTPReceiver. This class can best be described as the session's state machine controller. It retains all the knowledge about the state machine's possible transitions. These transitions are usually triggered by the received segments passed to the class from the session through its receiveSegment method, which is also the class's only entry point.

For a segment to reach this point, it must have passed the checksum and addressing tests performed by the protocol. Therefore, the method starts by waking up the session from its keep-alive state. After that, a validation check is performed on the segment. Once validated, the segment's basic information and extended options are extracted from the header before inspecting the segment's type. If it is an RST segment, the receiver drops the session; otherwise it processes the segment based on its simple or composite type. XSTP allows segments to combine certain types together to save network bandwidth by reducing the number of transmitted segments. However, these composite types are processed in a certain logical order that preserves 
the semantics of the protocol.

For each segment type, the receiver defines a handler method. Each method is passed as much information from the segment as it needs. The receiver also performs at least one or more of the following actions: trigger a state transition, order a certain response segment and ask for a certain post-action. While state transitions are executed right away by each handler, responses and post actions are collected first from all handlers then checked for sanity before being processed. A request to transmit the composite response segment is first forwarded to the sender object, followed by executing the requested post actions. The currently supported actions include one to keep-alive and another to notify the upper protocol of the peer's closure. Finally, since handling the received segment can affect the sender's state, a refresh to that state is performed.

Another major responsibility of the XSTP receiver is data assembly and presentation to upper protocols. While an STP session presents data asynchronously by appending the data to an STP session socket's receive buffer, an XSTP receiver presents the data synchronously by calling the upper protocol's $x$ Demux method. It is then up to the application protocol to consume the data directly or cache it for delayed consumption. When a data segment arrives, the receiver's data segment handler applies some semantic tests to it. Depending on the results of these tests, the segment is either ignored, presented, or cached in the receive cache. The cache 
is basically a sorted list of out-of-sequence segments. If the cached segment is also the next expected segment, the receiver checks whether it fills the first gap in the cache. If it does, a new data message is created to hold all the presentable data from the segment and the cache and is passed up to the upper protocol. If the segment is not the next expected one, the receiver just copies it to the cache in the appropriate order.

As in STP, the XSTP receiver has a responsibility to send USTAT notifications to the session's peer when new segment gaps are discovered. The reported segment gap is described by its lower and upper sequence numbers. However, in a network where packet reordering is a possibility, the receiver can be configured to delay this notification up to a number of further segments. If meanwhile the receiver discovers a new gap, it reports the first gap immediately and starts tracking the new one. Moreover, unlike STP, the XSTP receiver does not require the gap to be totally intact when it gets reported. Instead, the receiver allows reporting gaps that are partially filled. This process ensures that whatever remains from the gap is promptly reported and not ignored.

The final responsibility of an XSTP receiver is to process acknowledgment segments. The receiver uses the segment timestamp to make sure it only handles newer segments. When a POLL segment is received, the receiver requests the transmission of a STAT segment. This segment contains a gap report constructed by traversing 
the receiver's cache and creating a gap list. A gap is easily recognized when two consecutive segments in the cache have non-consecutive sequence numbers. In addition to handling POLL segments, a receiver also processes arriving STAT and USTAT segments. The receiver first extracts the updated peer's status information from these segments. It then passes this information to the sender by calling the sender's updateFlow Control method. The sender uses this information to adjust its flow control strategy.

\subsubsection{Sender Class}

The sender class is called XSTPSender. This class is responsible for constructing and transmitting segment messages and implementing the session's flow control strategy. As mentioned, the session passes data messages, pushed down from the application session, to its sender object. It does this by calling the sender's pushDataMessage method. However, the sender does not transmit a data segment right away. Instead, it copies the message's data to a send buffer. If there is not enough space for the data

in the buffer, the message thread is suspended. It is later awakened when some data is freed from the buffer. As soon as new data is buffered, the sender's state also gets updated.

At any point in time, the sender is either transmitting, persisting or sitting idle. The sender enters the transmission state when there is enough data to transmit and there is room in the transmission window. The transmission window is governed by 
a window control strategy that controls the number of segments that are allowed to be transmitted at any point in time. This window is calculated as the minimum of the receiver's advertised window and the sender's congestion window. The sender's transmission rate is not based on the rate of arriving acknowledgments (self-clocking), but rather on a send timer. This timer paces the transmission uniformly across the round trip to minimize the chance of introducing huge bursts into the network. Whenever the transmission state is entered, the sender updates the total number of allowed data segments, the timer sending interval and the burst size (the number of segments to be sent back-to-back each time). However, if the number of allowed segments is below a certain configurable threshold, the sender transmits all of them in one burst. If the sender determines that no new transmissions are currently possible, it immediately stops the send timer.

In addition to data transmission, XSTP senders also send POLL segments periodically. These segments are sent to trigger peers to transmit back STAT segments containing acknowledgments for the received segments. The polling rate is a configuration parameter that should be set discretionally. The higher the rate, the faster the segments get acknowledged, but also the more acknowledgment overhead is incurred. To minimize that overhead, POLL segments are allowed to be piggybacked with data segment if they both happen to be scheduled around the same time for transmission. To implement this feature, both have to be scheduled by the same send timer. With 
each timer tick, the sender's attemptTransmission method is called to examine if a transmission of a data segment, a POLL segment or both is due. After that process, the sender reschedules the next transmission (if any) before restarting the send timer. The attemptTransmission method is a hidden entry point to the session, and is therefore also synchronized.

An XSTP sender transmits a data burst by creating data segments and forwarding them back to back to its session for transmission. All segments in a burst except the last one have the maximum allowed segment size. If a POLL is scheduled at the same time of a data burst, the POLL is piggybacked with the last segment of the burst and thus takes from its full size. The sender can also force a POLL to be piggybacked with a data segment if the sender has transmitted a certain configurable ratio of the send buffer since the last POLL. This process is done to expedite receiving acknowledgments and therefore rapidly freeing the send buffer. Since the send buffer is not segmented, the sender maintains a separate queue of unacknowledged segment entries called the send cache. Before transmitting any segment, the sender makes a new entry for this segment in the cache. The entry contains the segment's sequence number, data size, number of retransmissions and the last retransmission timestamp. This information becomes handy in the segment retransmission process.

Under certain circumstances the sender might not be able to transmit any new data segment. This situation can happen if a slow peer advertises a zero window to 
prevent a fast sender from overwhelming this slow peer with data segments. In this case, the sender exits the transmission state and goes into the persistence state. In that state, the sender suspends data transmission and periodically schedules POLL segments to request window updates from the sender's peer. Only after receiving a non-zero window does the sender go back to the transmission state. When the sender is neither transmitting nor persisting, it is in the idle state. Only when the sender is in this state can a session be safely closed. However, a session can force its sender to go into the idle state by calling its stop method. This process is usually done as a session is dropped. When the stop method is called, all the threads that are suspended on the sender's insufficient buffer condition are resumed and allowed to safely return.

To facilitate the transmission of any kind of segment, the sender exports the transmitSegment method. This method knows about all the header fields and the options needed by each kind of segment. After collecting all the needed information, the method creates a new PIX message, whose size equals that of the segment plus the lower protocols' headers. The transmitSegment method then copies the segment's information to the message before forwarding the message to the session for actual transmission.

An other responsibility of the sender is to manage the session's flow control strategy. This strategy is affected by feedback received from the session's peer. This 
feedback can either be in the form of a STAT or a USTAT segment. These status segments contain both cumulative and selective negative acknowledgments. The cumulative acknowledgment informs the sender about the receiver's next expected in-sequence segment. This allows the sender to advance the left edge of its transmission window and remove entries from the sending cache up to but not including that segment's entry. The sender also frees data from the send buffer that belongs to those acknowledged segments and releases any pending threads that are waiting for the send buffer to free. After that process, the sender opens its congestion window either exponentially or linearly depending on whether the sender is in the slow start or in the congestion avoidance phase of flow control respectively.

On the other hand, the status segment's selective negative acknowledgment is basically a gap report. The sender examines every reportedly missing segment and determines if these segments qualify for retransmission. The sender distinguishes gaps reported by STAT segments from those reported by USTAT segments. While missing segments reported by a USTAT can only be retransmitted once, those reported by a STAT can be retransmitted only if an RTT (subject to backoff) has elapsed since they were last transmitted. To retransmit a segment, the sender has to find its entry in the send cache. Using information in that entry, the sender finds the segment's offset and size in the send buffer and uses that information to create another copy of the missing data segment. After examining all gaps and retransmitting the required segments, 
the sender closes its congestion window by cutting it in half. Further retransmissions in the same window does not result in further closing of the congestion window, since these retransmissions are probably caused by the same loss event. After closing the window for the first time, the sender exits the slow start phase and goes into the congestion avoidance phase. Once there, the sender never goes back to slow start again unless it has been idle for some time (a configuration parameter). 


\section{Chapter 4}

\section{XSTP-Probing Mechanism}

\subsection{Motivation}

In a typical heterogenous network, these exist many kinds of error conditions that widely vary in their nature. One of the classic problems of standard transport protocols in these networks is their inability to detect and effectively react to such different error conditions. The basic assumption made by those protocols is that network congestion is the cause of all perceived errors. However, in a typical LEO satellite access network there can be some other types of error conditions including bit corruption, handoff and limited comectivity. These non-congestion link-related errors are systemically perceived as congestion-related by those unaware transport protocols. Unfortunately, engaging congestion control in these cases hampers throughput and leaves the session too conservative even for moderate levels of link errors. Also, based on the used congestion control tactics, there can be further increase in the level of segment retransmission, which usually translates into more increases in energy 
expenditure.

Unfortunately, the STP protocol [Kat99, HK97] inherits this congestion control bias from its ancestor protocols. Although the protocol can efficiently recover from multiple losses in the same round trip, its error recovery tactics can negatively affect its overall performance. Specifically, slowing down in response to link-related errors can hamper the effective throughput. However, the protocol was found to outperform the standard versions of TCP in the existence of high level of BER and when multiple losses are reported in the same round trip. In this chapter, a new error control strategy is proposed for STP to stretch the protocol's ability to adapt to the different error kinds found in LEO satellite access networks. The new strategy is implemented in XSTP: the protocol's new implementation in the PIX framework, introduced in the previous chapter.

The new strategy is based on a probing mechanism called XSTP-probing that is implemented as a configurable option in XSTP. The mechanism is triggered upon detecting a segment loss to assess the level of congestion in the network. If congestion is detected, the mechanism responds by invoking congestion control; otherwise it resumes with Immediate Recovery ${ }^{1}$. The mechanism also adapts to the level of error in the network by suspending new data transmission and by striving to send

\footnotetext{
${ }^{1}$ Immediate Recovery restores the congestion window to the same level as before probing. [TB00]
} 
only in windows of error-free connection. The probing mechanism is modelled after an earlier one, proposed by Tsaoussidis and Badr in the context of TCP called TCP-probing [TB00]. The remainder of this chapter explains the probing mechanism mainly by comparing it to TCP-probing. An attempt to highlight how differences between the two mechanisms can positively or negatively affect the performance is also made. However, it is important to realize that an empirical comparison of the two mechanisms is not performed in this thesis and therefore all comparison remarks are not conclusive but rather argumentative. The main reason for that is unsuitability of TCP-probing to be directly implemented in XSTP due to lack of sufficient assumptions like the availability of the ACK timeout concept. Alternatively, an extra effort is needed to implement the TCP protocol and its probing mechanism as well in the PIX framework to be used for comparison with XSTP-probing. Since this is beyond the time frame of thesis, it is left as a future work.

\subsection{Description}

The goal of any error control strategy is to adapt the sender's transmission rate to the varying error conditions in the network. This goal is usually accomplished by taking an aggressive stance when the error is found to be transient and a conservative one when it is more persistent. The XSTP-probing mechanism is no exception. In fact, this mechanism goes even further by probing the connection for possible error free conditions and only transmitting in those windows. The mechanism accomplishes 
that task by suspending new data transmission upon detecting a loss and initiating a probing cycle to collect RTT statistics on the connection. The mechanism then compares these RTT statistics to the RTT estimate available when the loss was discovered. Interestingly, the duration of that probing cycle is proportional to the level of error in the network which helps the connection sit out the error conditions. After the cycle is finished and if congestion is detected by proliferating RTTs, congestion control is immediately invoked. Otherwise, transmission levels are restored without taking any action. Finally; reportedly missing segments are immediately retransmitted. Figure 4.1 highlights the basic algorithm of the XSTP probing mechanism.

Unlike the TCP-probing mechanism, which introduces changes to both the sending and the receiving ends of a connection, XSTP-probing is a sender-only mechanism. This property simplifies the implementation and facilitates the deployment of the mechanism to a network with nodes running an older version of the protocol. Also, while TCP-probing introduces several new segment types and their associated states, XSTP-probing leverages the unique semantics of XSTP and does not introduce any new segment types. In fact, XSTP-probing reuses the polling cycle of XSTP, which is the protocol's low frequency acknowledgement mechanism, as its probing cycle. Specifically, the POLL segment becomes the probe and the STAT segment becomes the probe acknowledgment. Fortunately, the XSTP receiver is kept unaware whether the received POLL is a probe or just a normal POLL. Also unlike TCP-probing, the 


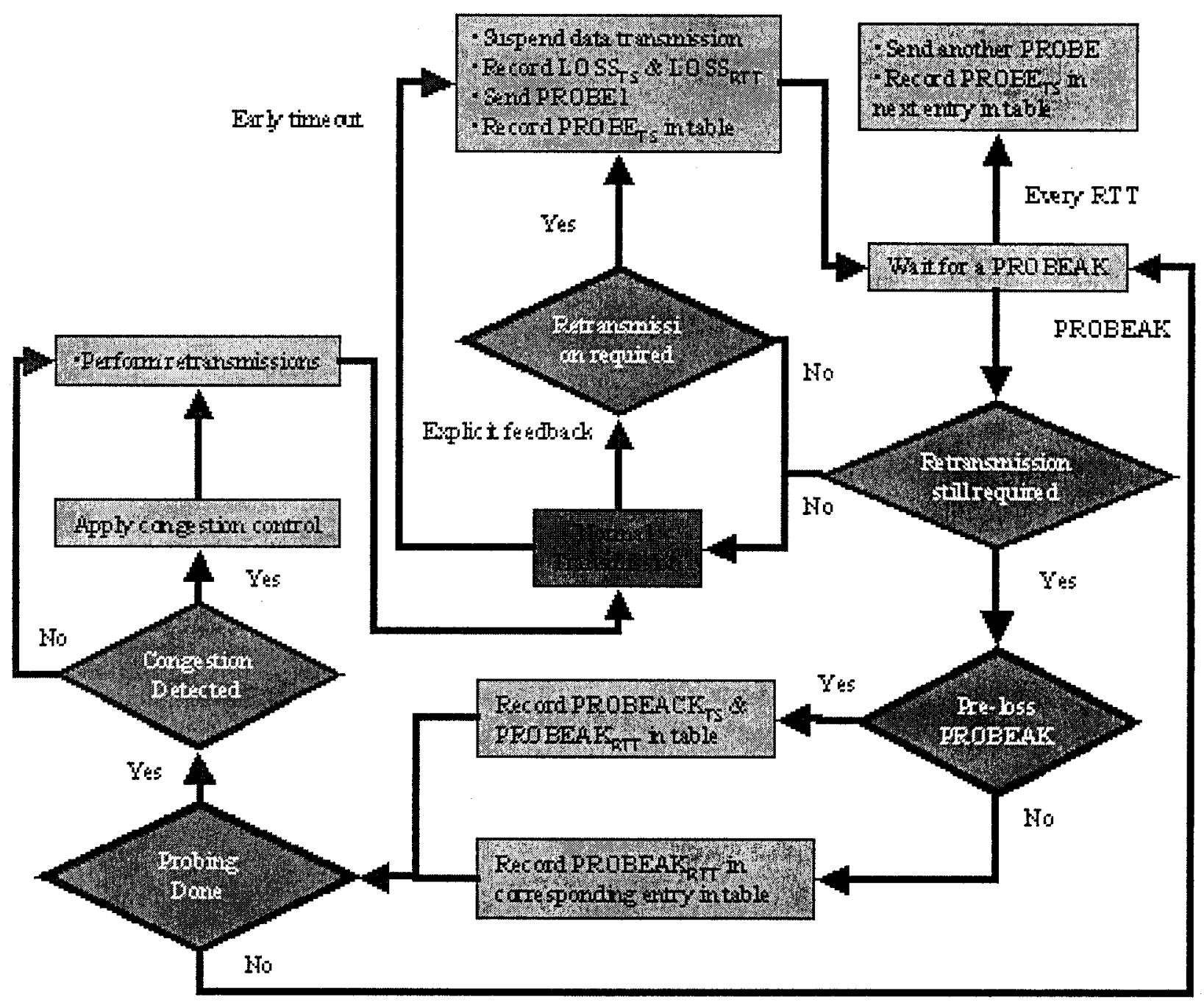

Figure 4.1: A flow chart of XSTP probing mechanism 
mechanism does not introduce several states to track the progress of the probing cycle. That fact makes XSTP-probing more scalable in terms of its ability to configure the probing cycle with different numbers of probing exchanges.

Furthermore, the mechanism gets triggered when a loss is discovered either implicitly or explicitly. The implicit method is called early timeout and is implemented by detecting a break in the POLL/STAT segment interleaf. To explain, XSTP senders transmit a configurable number of POLL segments every round trip. After the first round trip, STAT segments start to arrive at the sender. The rate of arriving STAT segments becomes similar to the rate of leaving POLL segments, producing an interleaved pattern of a sent POLL followed by a received STAT, as depicted in Figure 4.2. If either a POLL or a STAT is dropped in the connection, the session detects a break in the interleaf pattern within a maximum of one RTT and a minimum of RTT / POLLS_PER_RTT, as shown in Figure 4.3. This depiction is in contrast to TCP-probing; where a timeout usually spans multiple RTTS.

On the other hand, the explicit loss detection method relies on receiving feedback from the receiving session in the form of either a STAT or a USTAT segment. However, it is only after validating the gap reports in these segments and finding at least one segment worthy of retransmission that the probing mechanism gets triggered. This condition protects the mechanism from being triggered prematurely as a result of an invalid alarm. This method can be contrasted to TCP-probing's three DUPACK 


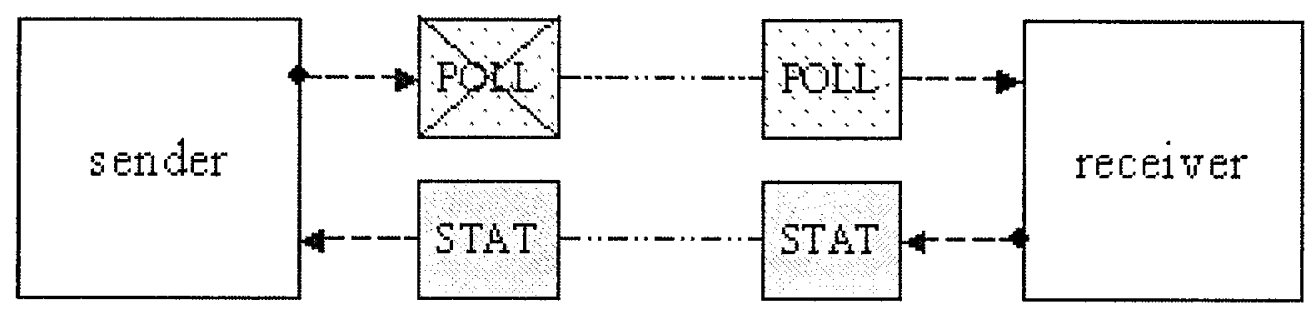

(a) Bya dropped POLL

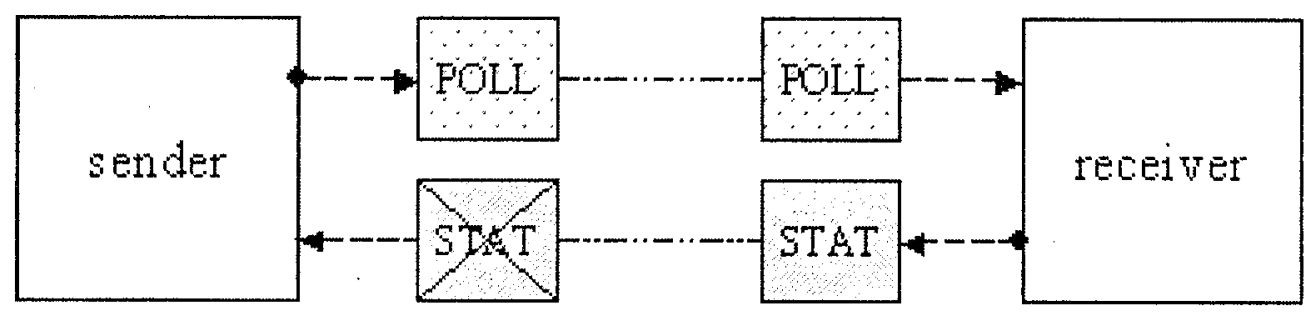

(b) Bya dropped STAT

Figure 4.2: Breaking the POLL-STAT interleaf

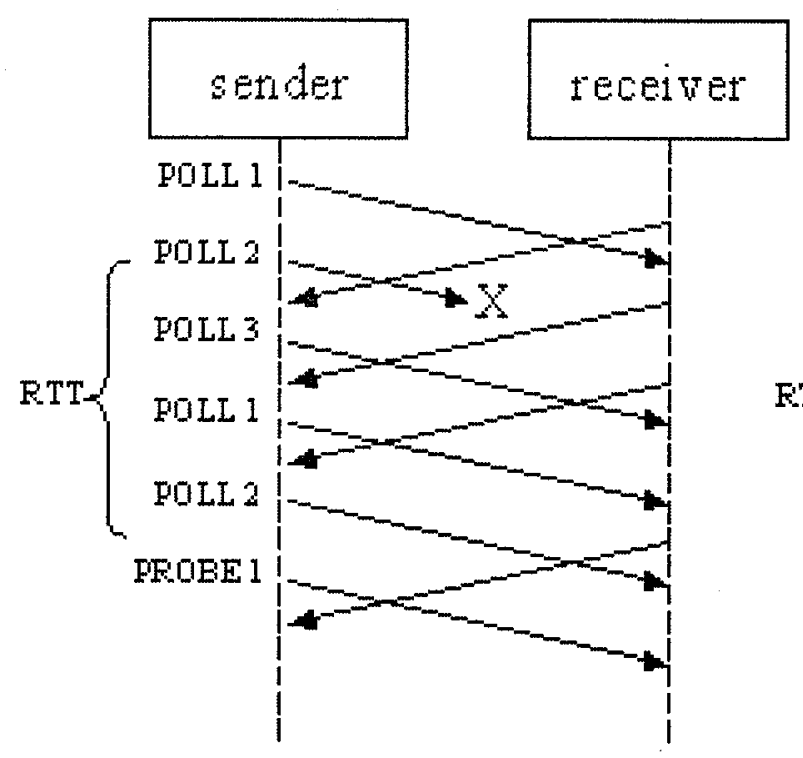

(a) Bya dropped FOLL

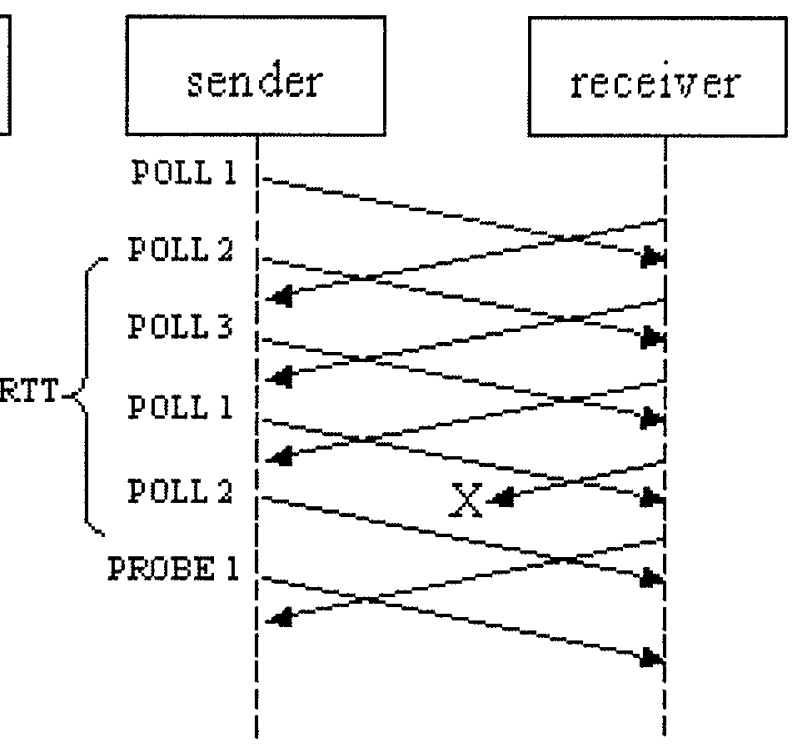

(b) Bya droped STAT

Figure 4.3: Triggering of the probing mechanism by an early timeout event 
heuristic, which is only a best effort and may lead to premature probing.

As the probing mechanism starts up, the session goes into probing mode. This mode supersedes the current state in the sender. Again, there is a contrast to TCPprobing going into the newly introduced "PR_ X_ SENT" states. After that event, the mechanism suspends new data transmission and records the current timestamp and RTT estimate (loss.TS and loss.RTT). Only then, the probing cycle becomes ready to be initiated. Furthermore, a fundamental difference between TCP-probing and XSTP-probing is not in the way their probing cycles are defined but in the way the cycles are implemented. Both mechanisms define the probing cycle as a number of consecutive exchanges of probes and their acknowledgements. For the sake of simplicity, that number is considered to be two (as in TCP-probing) for the remainder of this chapter.

In the case of TCP-probing, a complete cycle is labelled with a version number that is stored and reflected by all segments (two probes and two probe acknowledgments) participating in that cycle. Only after the first probe exchange is successfully completed, does the other exchange start. If any segment in that cycle gets dropped, the whole cycle is abandoned and a new one is initiated with a new version number. The mechanism detects the loss of a segment by means of a timeout (set to the currently estimated RTT). On the other hand, XSTP-probing follows its protocol in not using a timeout event. Rather, the mechanism sends a probe segment every RTT 


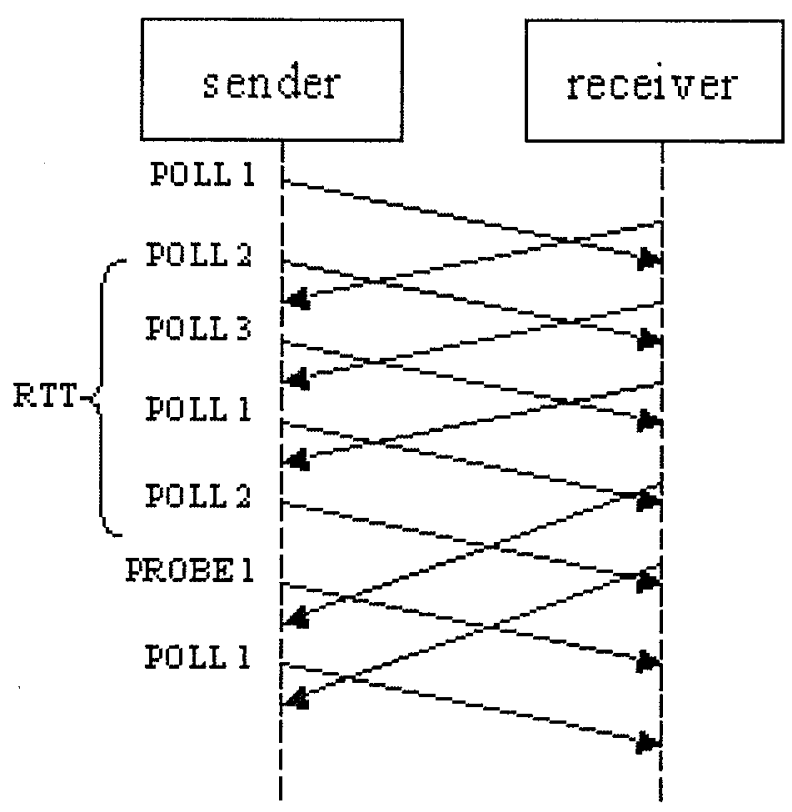

Figure 4.4: Triggering of the probing mechanism by a false early timeout event

irrespective of whether a probe acknowledgment is received or not. The advantage becomes clear when the RTT gets a little extended (a common phenomenon in LEO satellite links where the RTT experiences moderate variations). In this case, the previous exchange is not ignored but is rather given more time (up to one more RTT) to complete. If after that time it is still not completed, a new exchange begins and the old exchange becomes obsolete (since it is followed by two new probe exchanges). This situation is depicted in Figure 4.4, where PROBE1 gets transmitted when no stat arrived since the last POLL.

The XSTP-probing mechanism implements this strategy by defining an ordered 
map between the sent probe's (POLL) timestamp and its corresponding acknowledgment's (STAT) RTT measurement. Whenever a probe is sent, its timestamp is reordered in the next empty entry in the probing map. Also, whenever a valid probe acknowledgment is received, its RTT measurement is saved in the corresponding entry in the map. The probing cycle does not complete until two consecutive entries in the map get filled with RTT measurements. It is important to mention that the probing map has a constant size that is set as a configuration parameter. The map also has a policy of deleting the oldest entry to make room for new probe entries; hence the map size should be set in proportion to the expected error levels in the network. Figure 4.5 illustrates different phases of a typical probing cycle as they occur in the network. Figure 4.6 shows the corresponding stages of the cycle as reflected in the probing map.

Three more salient points exist in the previous strategy. The first point is related to arriving probe acknowledgements that bring back empty gap reports. This event is usually taken by the mechanism to indicate that probing is started prematurely due to packet reordering or a false early timeout event. In this case, the mechanism immediately self terminates, turns the probing mode back off and restores new data transmission at the previous levels. The second point relates to arriving probe acknowledgments (STAT segments) that do not correspond to existing probes in the 
(b)

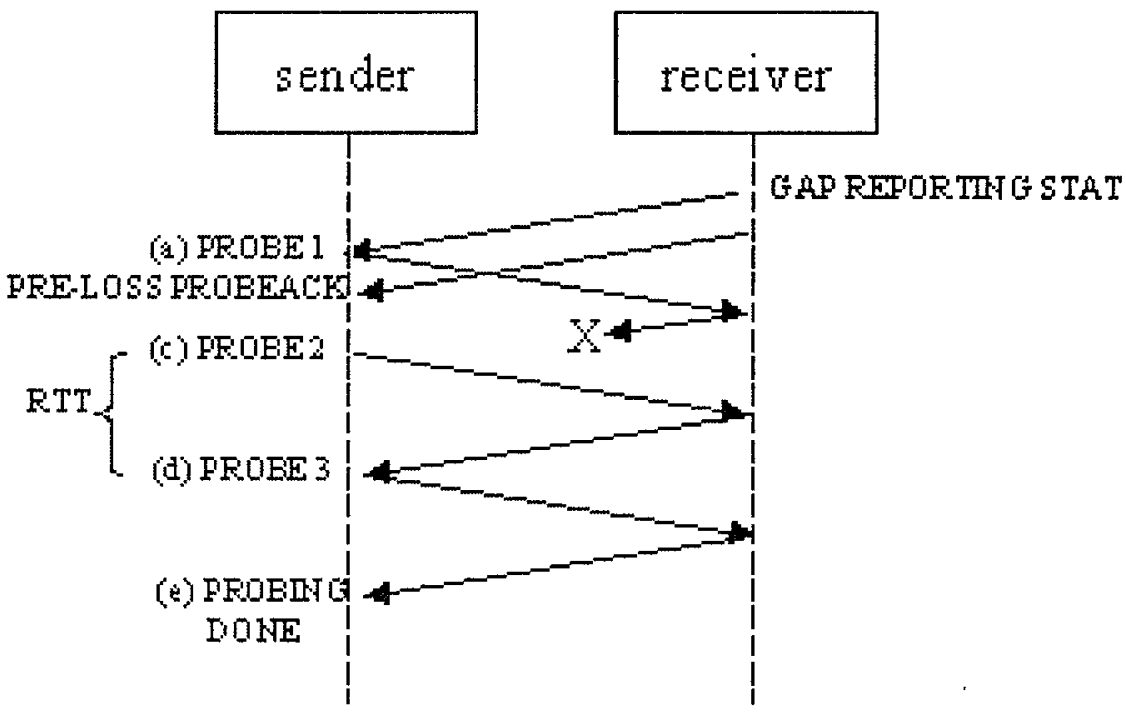

Figure 4.5: Phases of a probing cycle as it happens in the network

\begin{tabular}{|c|c|}
\hline \multicolumn{2}{|c|}{ Frobinc Table } \\
\hline PFOEE TS & PFOEE RT T \\
\hline & \\
\hline $\mathbf{1}^{\text {Ex }}$ Ts & \\
\hline & \\
\hline & \\
\hline
\end{tabular}

\begin{tabular}{|c|c|}
\hline \multicolumn{2}{|c|}{ Fobine Table } \\
\hline PROEE TS & FFOEE RTT \\
\hline Pre-Loss TS & Pre-Loss RTT \\
\hline In $^{\text {TS }}$ & \\
\hline & \\
\hline & \\
\hline
\end{tabular}

(b) After recering po-loss STAT

\begin{tabular}{|c|l|}
\hline \multicolumn{2}{|c|}{ Frobine Table } \\
\hline PROEE TS & FROEE RTT \\
\hline FTu-Lose TS & FTs-Lose RTT \\
\hline 1n TS $^{\text {nt }}$ & \\
\hline $2^{\text {nd }}$ TS & \\
\hline & \\
\hline
\end{tabular}

(c) Bfter one RTT

(a) Start of mobing

\begin{tabular}{|c|c|}
\hline \multicolumn{2}{|c|}{ FTobing Tsble } \\
\hline PEOEE TS & PFOEE RTT \\
\hline FTe-Loss TS & Fre-Loss FTT \\
\hline $\mathrm{l}^{\mathrm{sh}} \mathrm{TS}$ & \\
\hline 2 ad TS & $2^{n d} \mathbf{R T}$ \\
\hline $3^{\text {rd }} \mathrm{TS}$ & \\
\hline
\end{tabular}

(d) After two RTTs

\begin{tabular}{|c|c|}
\hline \multicolumn{2}{|c|}{ Probing Tsble } \\
\hline PEOBE TS & PROEE RTT \\
\hline PTe-Loss TS & FTe-Loss RTT \\
\hline $1^{\prime 4} \mathrm{TS}$ & \\
\hline $2^{\mathrm{d}} \mathrm{TS}$ & $2^{\text {ad }}$ RT T \\
\hline $3^{1 d} T S$ & $3^{\text {rd }}$ RTT \\
\hline
\end{tabular}

(e) After three RTTs

Figure 4.6: Phases of a probing cycle as reflected in the probing map 
map. This scenario usually appears in one of two situations: either the acknowledgments took so long to arrive that their probe entries got deleted from the map, or these acknowledgments correspond to POLL segments that had been sent before probing was started (their timestamps are smaller than loss.TS). While the mechanism ignores the acknowledgments in the former case, it records them in the map in the latter. Actually, during the first RTT of probing, the mechanism reserves an entry in the map (usually the first) to those kinds of acknowledgments. The rationale to consider those acknowledgments is their being close enough in time to the error (sent during the same RTT containing the error) that their feedback provides invaluable information about the network condition at that time. The mechanism records the acknowledgments' timestamps and RTT measurements in the reserved entry in the map. Since XSTP normally sends multiple POLL segments per RTT, the probing mechanism can receive several pre-loss acknowledgments. In this case, the mechanism overrides the reserved entry in the map with every arriving acknowledgment. Interestingly, this ability to handle pre-loss probes can allow the probing cycle to finish much quicker (in around one RTT) if the first probe acknowledgment also made it on time (since two exchanges are required). The third and final point is related to USTAT segments. Although they are used as explicit triggers to the probing mechanism, the segments are totally ignored while the probing mode is on. Their gap reports, usually repeated by consecutive STAT segments, are processed only after probing is done. 
One very important side of the probing mechanism is the decision making criteria at the end of the probing cycle to determine the probable cause of the error. Although many possible heuristics can be used, the same simple one introduced by TCPprobing is adopted by XSTP-probing. This heuristic compares the two probes' RTT measurements to the loss.RTT. If both probes are less than or equal to the loss.RTT, congestion is "not" detected and the error is considered link-related. Otherwise, congestion control is applied. However, in some cases, the RTT can moderately vary over the round trips (for example due to LEO satellite mobility). In this case, the varying RTTs can confuse the probing mechanism into thinking that the RTT has extended due to congestion. For this scenario, we define a configurable RTT tolerance parameter (ratio) in the probing algorithm to smooth out the effect of that phenomena on the measurements. This parameter should be set based on the expected RTT variance in the network.

As mentioned, the mechanism invokes congestion control as soon as it detects congestion. However, the specific congestion control measure depends on how long the probing cycle takes to finish. If the cycle is long enough to reach XSTP's threshold for idle transmission, the sender goes back to slow start; otherwise the congestion window is closed by the congestion avoidance algorithm (the window gets halved). This congestion control logic is in contrast to TCP-probing where the sender goes back to slow start if probing is triggered by a timeout event. If the timeout later 
turns out to be inaccurate, the connection becomes needlessly overconservative. After probing is done, the missing segments reported by the last probe acknowledgement (STAT) are retransmitted and the normal polling rate is restored.

\subsection{Design}

The XSTP-probing mechanism is implemented as a configurable option on the XSTP session. The mechanism is modelled as a class called XSTPProbing. Each XSTP sender object owns an instance of that class. This instance is created with three configuration parameter's: the maximum number of trackable probe exchanges $M A X_{-} P R O B E S$;

the number of requested probe exchanges $R E Q_{-} P R O B E S$; and the RTT tolerance ratio $R T T$ TOLERANCE. The probing object's local state contains a loss descriptor and a probing map. The loss descriptor consists of a loss timestamp (loss.TS) and a loss round trip time (loss.RTT). The probing map, whose size is set to $M A X_{-} P R O B E S$, is implemented as a circular array of probing entries containing the probe's timestamp and its corresponding RTT measurement. The entries are sequentially ordered by the probe's timestamp. The probing object also keeps an activation flag, which is initially set to false.

A sender starts the probing mechanism by calling the probing object's activate method with the current timestamp and RTT estimate. The object uses these values to initialize its loss descriptor before turning its activation flag on. Once the object 
is activated, the sender enters the probing mode, which means suspending new data transmission by closing the data sending interval, changing the polling rate to one POLL per RTT and disabling further RTT backoff. The sender then initiates a probing cycle, in which it sends a probe each round trip. For each probe, the sender records the probe's timestamp by calling the probing object's recordProbe method. This method puts the timestamps in the next entry in the probing map. When all entries get filled, the probing object starts overriding older entries. It is important to mention that the first probe's timestamp goes into the second not the first entry in the map. The first entry is initially reserved for potential pre-loss probes.

Whenever a probe acknowledgment (STAT) arrives, the sender updates its congestion window by examining the segment's cumulative acknowledgment. After that, the sender examines the segment's gap report. If the report is empty, the sender immediately aborts the probing mechanism by calling the probing object's deactivate method. This method resets the measurement map and loss descriptor before turning its activation flag off. However, if some segments are reported missing, the normal gap validation is performed. If at least one segment is determined worthy of retransmission, the STAT is considered a valid probe acknowledgment. In this case, the sender measures its RTT and calls the probing object's recordMeasurement method with both the acknowledgment's timestamp and RTT values. 
This recordMeasurement method starts by comparing the acknowledgment's timestamp to that of the loss descriptor. If the timestamp is smaller, the acknowledgment is considered a pre-loss probe acknowledgment and both its timestamp and RTT values are stored in the first entry in the map. If the received acknowledgment is not the first pre-loss acknowledgement, the method overrides the older data in the first entry (usually pre-loss acknowledgements stop arriving after the first post-loss probe acknowledgment is received). However, if the acknowledgment's timestamp turns out to be greater than the one in the loss descriptor, the method looks up the acknowledgment's corresponding timestamp entry in the map and records its RTT in the appropriate corresponding slot.

Finally, the method checks if there are $R E Q_{-} P R O B E S$ consecutive filled entries in the map. If there are, the method returns true to signal back the end of the probing cycle by returning true; otherwise the method returns false. If the method returns positively, the sender calls the probing object's is CongestionDetected method, which implements the congestion detection heuristic mentioned in the previous Section. The method first applies the tolerance ratio RTT TOLERANCE to the RTT measurements (only those consecutive REQ PROBES measurements that ended the probing mode). The method then compares the RTT measurements to the loss.RTT. If any one of the measurements is greater than the loss.RTT, congestion is signaled 
by returning true; otherwise false is returned. The sender then terminates the probing mechanism by calling the probing object's deactivate method. If congestion is detected, the sender closes its congestion window by the congestion avoidance algorithm; otherwise the window is left alone. The sender then concludes by retransmitting the missing segments, restoring the polling rate and the resuming new data transmission. 


\section{Chapter 5}

\section{Simulation and Performance Metrics}

The previous chapters introduced the new design of the XSTP protocol along with its probing mechanism implemented in the PIX framework. The mechanism establishes an adaptive error control strategy for the protocol. In a LEO satellite access network, errors can vary in nature from congestion-related to link-related. They also vary in their intensity (frequency and duration) from transient to more persistent. Using simulation, the new XSTP-probing mechanism is tested in various error conditions and its effect on the protocol's performance is quantified. This chapter describes the simulation environment and configuration, enumerates the metrics used to evaluate the effect on performance and finally elaborates on the different test cases under study. 


\subsection{Simulation Environment}

The design of the XSTP protocol is realized in the PIX framework. The implementation is done in a recent distribution of the Linux $\mathrm{OS}^{1}$ using $\mathrm{C}++$. XSTP is configured as part of a communication protocol stack that is implemented exclusively in PIX for this simulation. Advantages of that setup include the ability to run the whole simulation in user- mode outside the kernel, making the process of debugging and logging much easier. The protocol also gets tested using its original code, instead of having to re-implement the code in some simulator's pseudo-language, which helps keep its semantics more intact. The obvious downside here is the extra effort of implementing a simulator and the lack of a comparative set of results. However, this downside will be remedied by offering this simple simulator for further study by the research community.

As part of the simulation framework, three more PIX protocols are implemented: an application protocol; a link protocol; and a protocol encompassing an error generating model. The application protocol (APP) is a simple bulk data streaming protocol. Client sessions of that protocol act as data sources by streaming large files from a persistent storage across the network using a configurable speed. Server sessions, on the other hand, act as data sinks by serializing the file streams back to the persistent storage. Bulk data transfer is favored here since it is the best traffic pattern to study

\footnotetext{
${ }^{1}$ Mandrake Linux version 8.0 is used
} 
the effect of errors on the efficiency of transport protocols. The long and continuous flow of data allows the transport protocol to demonstrate the full capabilities of its error control strategy. Configuration parameters for this protocol's sessions include the file size, the chunk size and the streaming rate.

The simulator's link protocol is called the Queue Link Protocol (QLP) since it is based on the POSIX message queues. Sessions of that protocol represent nodes in a simulated virtual network on a single physical machine. Each session is configured with a message queue identifier representing the node's link interface address. A session is also configured with a simple static forwarding table containing a map of possible destinations to their corresponding message queue identifiers. Using this protocol, it is possible to simulate networks of various topologies. Figure 5.1 shows how QLP sessions can simulate virtual network nodes. It is important to mention that QLP is a non-reliable protocol, which means it does not attempt to recover from link errors locally. QLP is also not transport-aware, which means it does not attempt to inform the transport protocol of link-level events (handoff for example).

The third and final simulation protocol is implemented to use an error modelling algorithm. The protocol is called the eXtended Delay and Drop protocol (XDELDROP). It is based on a similar protocol for $\mathrm{x}$-Kernel ${ }^{2}$ called VDELDROP, proposed by Tsaoussidis and Badr [TB00]. The main rationale of the used algorithm

\footnotetext{
${ }^{2} \mathrm{x}$-Kernel is another function-oriented protocol implementation framework [HP91]
} 


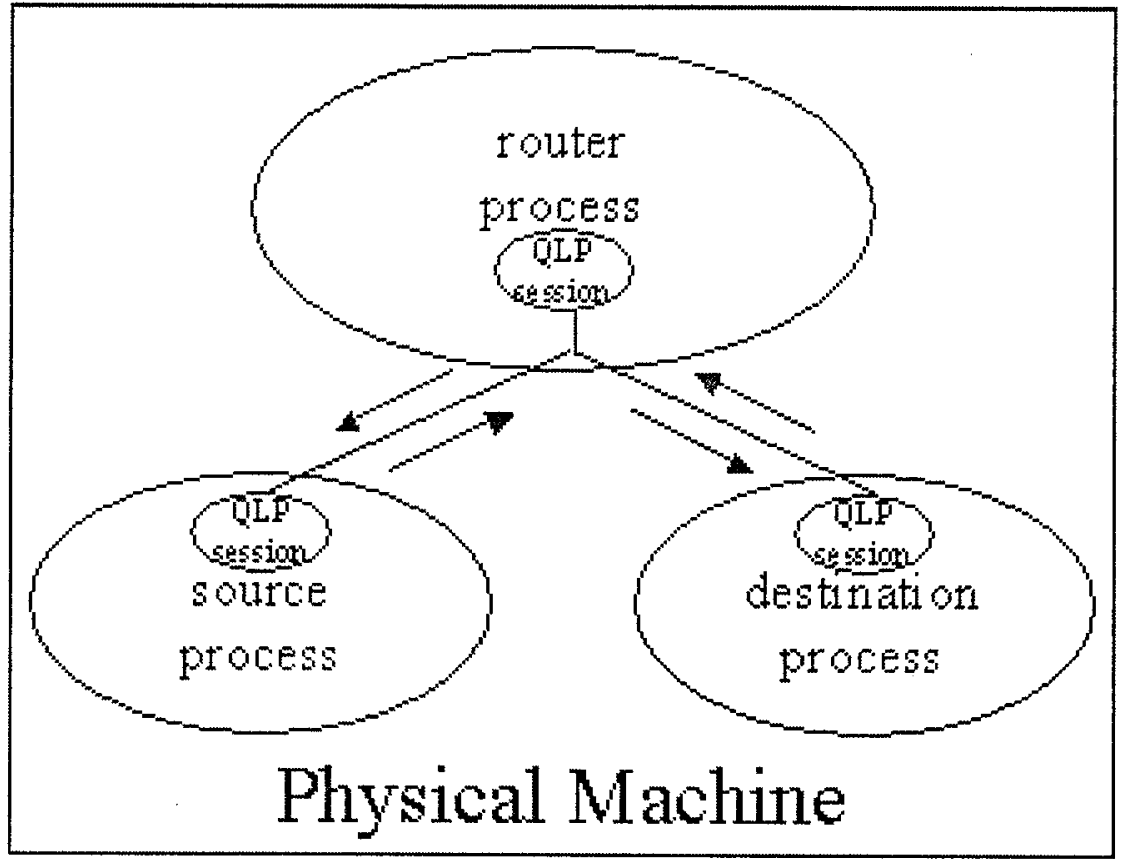

Figure 5.1: Simulating a virtual network using QLP 
is to model the delays and drops of packets in a heterogeneous network (like a LEO sátellite access network). Each session is modelled as a continuous-time Markov chain with two states. Each state has three configurable parameters: a mean sojourn time $t_{i}$ to model the persistence of the state; a dropping rate $r_{i}$ to model the severity of the error condition; and a delay range $d_{i}^{\min }$ to $d_{i}^{\max }(i=1,2)$ to model the prevailing end-to-end delay in the network, as shown in Figure 5.2.

When a session enters a particular state, it stays an exponentially distributed amount of time with mean $t_{i}$ before switching to the other state. During that time, the session receives incoming packets and either drops them with a probability $r_{i}$, or forwards them after applying the minimum delay $d_{i}^{\text {min }}$ or the maximum delay $d_{i}^{\text {max }}$. The choice of either delay is random with a uniform distribution but is maintained during each state. The minimum delay usually corresponds to the normal one way delay across the network when it is uncongested, while the maximum delay corresponds to some level of network congestion delay. The XDELDROP protocol inherits VDELDROP's insensitivity to packet size with respect to the dropping rate. However, unlike VDELDROP, the former protocol is configured above the link layer in intermediate nodes (router nodes) rather than below the transport protocol at the terminal nodes. Although the two cases are functionally equivalent, it is more logical to place sessions of that protocol in intermediate nodes since these sessions are more tied to network links than network nodes. In this setup, many terminal nodes can 


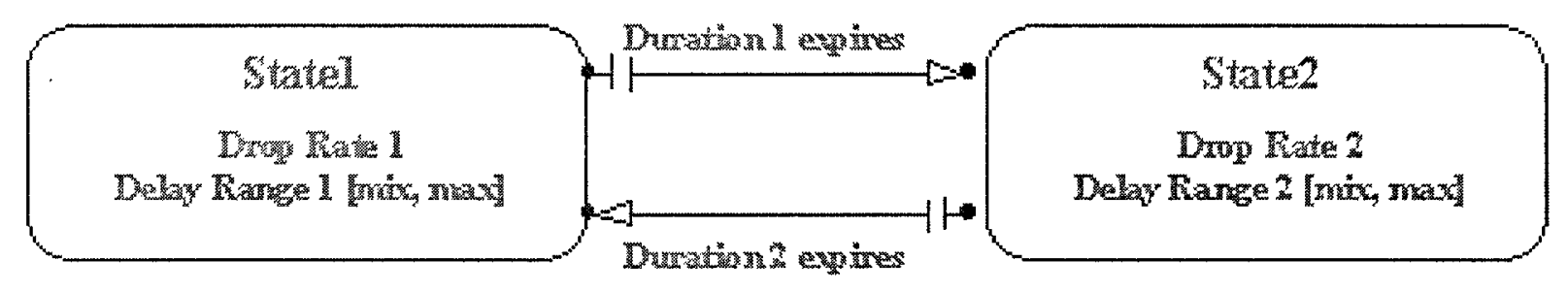

Figure 5.2: The two-state Markov chain in the XDELDROP protocol

share the same error session and be exposed to the same conditions simultaneously. Also, by installing different sessions of that protocol in multiple intermediate nodes, the effect of links with different error characteristics can be simulated.

\subsection{Simulation Configuration}

In this simulation, a simple LEO satellite access network is configured. In essence, this network consists of a source terminal, a destination terminal and a network path that starts with a LEO satellite link and possibly spans several other satellite and non-satellite links. The configuration used to model this network is shown in Figure 5.3. There are essentially three network nodes: a source node; a router node; and a destination node. In the source and destination nodes, a communication suite consisting (from top to bottom) of the application protocol APP, the transport protocol XSTP and the link protocol QLP is installed. In the router node, a suite consisting of the error generating protocol XDELDROP directly over the link protocol QLP is installed. Each node is run in a separate multi-threaded heavy-weight process on the 


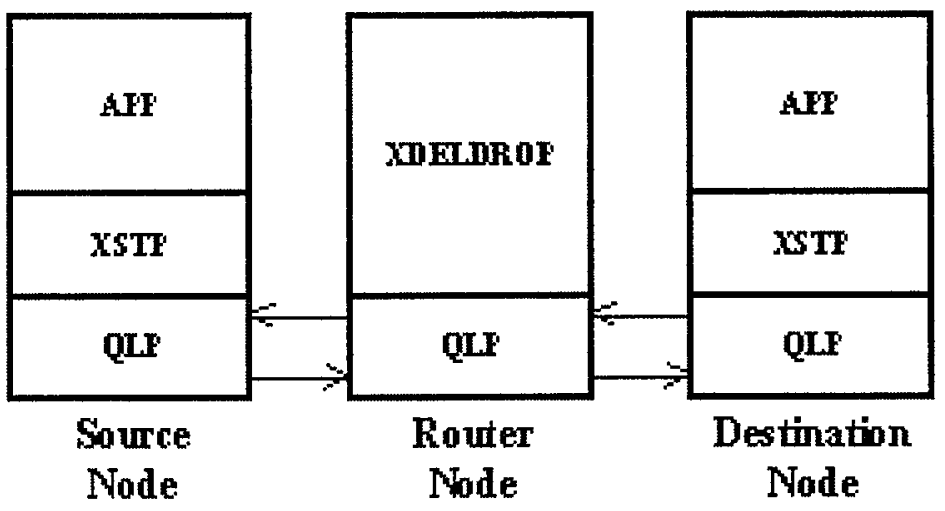

Figure 5.3: The general simulation configuration

same physical machine.

The application session in the source node is configured to be a data source, streaming a sufficiently large (10,000,000-byte) file from disk. In the destination node, the application session is configured to be a data sink, serializing the file data stream back to disk. The source session is configured to stream in chunks of 1000 bytes each, which is equivalent to the XSTP session's maximum segment size (MSS). This configuration is chosen to avoid any buffering delays and to neutralize some XSTP algorithms such as the Nagle's algorithm ${ }^{3}$. The flow of data to the XSTP session is also configured to be continuous with no delay. Furthermore, a single connection from the source to the destination is run in each test. The reason for not having competing connections is to avoid any unpredictable conditions with distorting

\footnotetext{
${ }^{3}$ Nagle's algorithm is used by transport protocols to delay sending small segments for some time until previous acknowledgements are received.
} 
effects on the measurements. Another reason relates to fact that the XDELDROP session in the router is already modelling the effect of a complex network, which usually contains competing traffic, by simulating its delays and drops. Therefore, the addition of explicit competing traffic is not really needed in this case.

Also, the QLP sessions are configured to provide a two-way network path between the source node and the destination node passing through the router node. This setup is done by configuring both terminal QLP sessions to have the router's session as their only destination. On the other hand, the router's session has two possible destinations; each goes back to one of the terminal sessions. Obviously, the QLP protocol mitigates the need for a network protocol since the routes are already preestablished.

Moreover, the benefit of the XDELDROP protocol is the flexibility to build a complex error model without the need to actually build a complex network model. The protocol achieves that flexibility by capturing the aggregate effect of different errors in a network path and modelling that effect as a simple drop and delay state-machine. An XDELDROP session, usually installed in the router node, can be configured to simulate all the desired errors in the network connection. As mentioned in the test cases below, different error conditions are simulated including a bit corruption condition, a handoff condition and a limited connectivity condition. Every one of these 
conditions is coupled with a congestion condition. Details of all XDELDROP configurations are explained in the test cases section.

Generally, the XDELDROP sessions are configured to clearly have two distinct states: an error-free state (also called the good state); and an error-prone state (also called the bad state). The only parameter that is fixed for both states throughout the tests is the packet forwarding delay range, which is set to a minimum of $100 \mathrm{msec}$ and a maximum of a $150 \mathrm{msec}$. The minimum value represents a hypothetical normal oneway delay when there is no congestion condition, while the maximum value indicates the same delay under a hypothetical congestion condition. The particular values for the parameters do not correspond to a specific real network topology but are rather set to distinguish a congestion from a non-congestion condition in any LEO satellite access network. A similar approach is taken for TCP-probing [TB00]. The reason for setting the maximum to be $50 \%$ more is simply to overcome an implementation limitation in the Linux kernel, where the accuracy of the "sleep" method on Intelbased systems tends to be unacceptable in the msec granularity. On the other hand, the values of the mean sojourn time and the dropping rates vary depending on the error condition desired to simulate in each case. However, in the good state a drop rate of zero is always assigned.

In general, an XDELDROP session is in either one of four states: no error condition; moderate congestion condition; link error condition; and severe congestion 


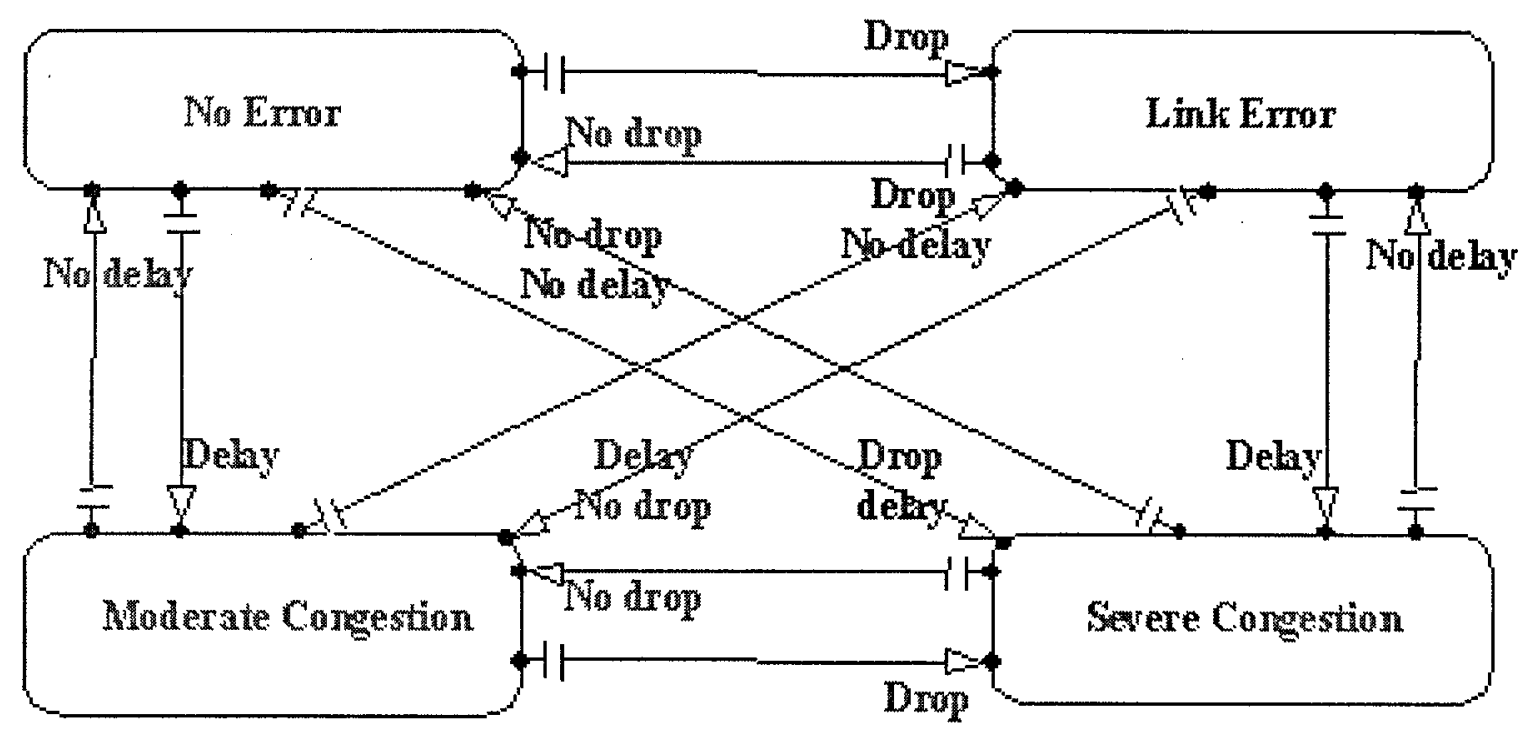

Figure 5.4: The four states of the simulated network

condition. The no error condition occurs when the session is forwarding all packets (good state) after applying the minimum forwarding delay. On the other hand, the link error condition takes place when the session starts dropping packets (bad state) while applying the minimum delay to forwarded ones if any. Similarly, the moderate congestion state occurs when the session is forwarding all packets (good state) after applying the maximum forwarding delay. The congestion becomes severe if the session starts dropping packets (bad state) and applying the maximum delays to forwarded ones if any. The state machine transitions are demonstrated in Figure 5.4.

In fact, the configuration of the XSTP session can greatly affect the overall performance of the connection. The source and sink sessions are configured similarly 
in the simulation. The sender and receiver buffer sizes are set to 64000 bytes, while the maximum segment size (MSS) is set to 1000 bytes, which leads to maximum window size of 64 segments. The polling frequency is also set to three per round trip and sending a POLL with the first burst is enabled. In addition, the USTAT sending threshold is configured to be three out-of-order segments. The initial congestion window is set to one segment size and the maximum burst size is set to eight segments. The probing option is also configured in the same way for all tests in the simulation. The maximum number of trackable probes is set to four probes, and the number of consecutive RTT measurements sufficient to finish the probing cycle is set to two measurements. Due to the mentioned sleep method's inaccuracy, the probing tolerance is set to $25 \%$ to normalize that effect. Ironically, this inaccuracy helps the modelling of a LEO satellite access network, since that inaccuracy introduces some variations in the RTT normally expected in a network with one or more LEO satellite links.

\subsection{Performance Metrics}

In the simulation, an attempt is made to follow the approach taken by Tsaoussidis and Badr in [TB00] and by Lahanas and Tsaoussidis in [LT01]. One of the relevant performance metrics in this context is the effective throughput ${ }^{4}$, which is defined as the average data reception rate (bits/sec) as seen by the data sink session and is

\footnotetext{
${ }^{4}$ This definition of effective throughput is used as in [TB00]
} 
calculated using the following formula:

$$
\text { effective throughput }=\text { original size/connection time }(\text { bit/sec })
$$

Another very important metric, particularly for battery-powered devices like LEO satellites and satellite modems is the level of energy expenditure during the connection. While there are plenty of factors to consider for the effective evaluation of energy expenditure, the focus is made on the transmission overhead metric ${ }^{5}$. This metric is defined as the percentage of extra bytes expended in the reliable transmission of the original data bytes. The transmission overhead is calculated using the following formula:

$$
\text { transmission overhead }=((\text { total size }- \text { original size }) / \text { original size }) \times 100(\%)
$$

A third metric that is also used is the throughput/overhead ratio. This metric is defined as the effective throughput achieved per one percent of expended transmission overhead. The ratio is calculated using the following formula:

throughput/overhead ratio $=$ ef fective throughput/total transmission overhead $($ bit $/ \mathrm{sec})$

This metric is particularly useful to measure the protocol's ability to manage the usual tradeoff between throughput and overhead.

To calculate these metrics, several statistics are collected in each test. A test is replicated twenty times (to smoothen out the statistical variations) with only one

\footnotetext{
${ }^{5}$ This definition of transmission overhead is used as in [TBO0]
} 
statistical data sample taken at the end of each test (after all data is sent from the source to the destination session). The mathematical average of the collected twenty samples is calculated along with their standard deviation and $95 \%$ confidence interval. The first statistic is the total connection time in seconds, which is enough to calculate the effective throughput. The other statistics are the retransmission overhead, the forward control overhead and the reverse control overhead (all in bytes). The sum of all those overhead values constitutes the total transmission overhead. However, it sometimes helps to look at individual components of the overhead to gain further insight. More details of the collected statistics can be found in Appendix B.

\subsection{Test Cases}

To properly evaluate the effect of the probing mechanism on the effective throughput and the energy efficiency of the XSTP protocol, several simulation tests are carried out. These tests are grouped into three main categories representing the three different satellite link error conditions under study. For each category, the XDELDROP session is configured to produce phases of that category's link error in addition to phases of congestion. This configuration permits the analysis of the mechanism's effect on performance for each type of error independently with congestion. Moreover, each individual test is basically a variation of the main theme of the category. The variation is either in the duration or the frequency of the error condition. Also, for 


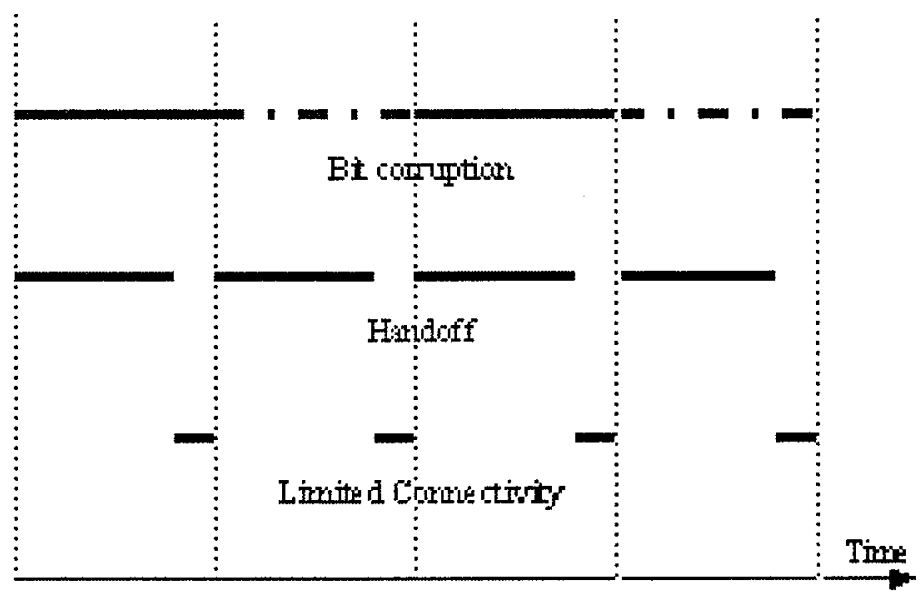

Figure 5.5: The three simulated link errors

each test two versions are configured: one with the XSTP session turning the probing mechanism on, and another one with the session turning it off. The different categories defined, also shown in Figure 5.5, are as follows:

\section{Bit Corruption Tests}

In this category the good and bad states have the same mean duration time.

While the good state has a drop rate equal to zero, the bad state's drop rate is varied from 0-50\% [TB00] and is the same for both directions. The specific test configurations as follows:

\begin{tabular}{|c|c|c|}
\hline Probing & Mean Duration (sec) & Drop Rate (\%) \\
\hline ON, OFF & 1,10 & $0,1,5,10,20,33,50$ \\
\hline
\end{tabular}

\section{Handoff Tests}

In this category, the good state is configured to have a zero drop rate, which 
makes it forward all packets in both directions. On the other hand, the bad state is configured to have a $100 \%$ drop rate, basically dropping all packets in both directions. The duration of the good state is larger than the duration of the bad state in this test. Different levels of handoff rates and duration (rendezvous) are tested by varying the mean duration time of both the good and bad state [LT01]. To simulate a required handoff rate and rendezvous combination, the bad state's mean duration is set to the handoff rendezvous and the good state duration is calculated as:

$$
((\text { rendezvous/rate }) * 100)-\text { rendezvous }
$$

The specific test configuration used are as follows:

\begin{tabular}{|c|c|c|}
\hline Probing & Handoff Rendezvous (sec) & Handoff Rate (\%) \\
\hline ON, OFF & $0.5,1$ & $1,3,5,10,15$ \\
\hline
\end{tabular}

\section{Limited Connectivity Tests}

In this category, the good state is configured to have a zero drop rate, which makes it forward all packets in both directions. On the other hand, the bad state is configured to have a $100 \%$ drop rate, basically dropping all packets in both directions. The duration of the bad state is larger than the duration of the good state in this test. Different levels of connectivity rate and duration (rendezvous) are tested by varying the mean duration time of both the good and bad state. To simulate the required connectivity rate and rendezvous combination, the 
good state's mean duration is set to the connectivity rendezvous and the bad state duration is calculated as:

$$
((\text { rendezvous } / \text { rate }) * 100)-\text { rendezvous }
$$

The specific test configuration used are as follows:

\begin{tabular}{|c|c|c|}
\hline Probing & Connectivity Duration (sec) & Connectivity Rate (\%) \\
\hline ON, OFF & 5,10 & $10,15,20,25,30$ \\
\hline
\end{tabular}




\section{Chapter 6}

\section{Simulation Results and Analysis}

In this chapter, the results of the simulation tests, defined in the previous chapter, are reported and explained. The chapter also presents an analysis of the results and highlights any interesting observations. Throughout the chapter, the XSTP session with the probing option turned off is referred to as XSTP-OFF. Similarly, the XSTP session with the probing option turned on is referred to as XSTP-ON. It is important to keep in mind that the XSTP protocol is already more tuned to carry out error control than the standard TCP versions. Katz and Henderson [Kat99, HK97] showed that the protocol can effectively recover from multiple errors (including link errors) in the same round trip. It is also interesting to mention that TCP-probing was only compared against the standard versions of TCP. Therefore, in contrast to TCP-probing, the performance gains achieved by the XSTP-probing mechanism are relative to a more extended protocol, which gives the results further significance.

For each test in the simulation, the total connection time and three kinds of 
transmission overhead are tracked: the retransmission overhead; the forward control overhead: and the reverse control overhead. While it is generally sufficient to observe the total transmission overhead, in some cases it helps to look at an individual overhead component to gain more insight. The complete measurement data along with their standard deviation and 95\% confidence interval are reported in Appendix B. This data is used to calculate three different metric values: the effective throughput; the total transmission overhead; and the throughput/overhead ratio. The analysis in the remainder of the chapter is outlined first by the error category ad second by the affected metric, both of which are defined in the previous chapter.

\subsection{Bit Corruption Tests}

In these tests, packets are dropped in both the good state and the bad state with a certain probability. On the other hand, packets surviving the drop are forwarded after being subjected to one of the two configured delays. The same error condition is applied to both directions of the connection at the same time. Usually, when packet drops are accompanied with extensions to the RTT, a congestion condition in the network, resulting from network over-buffering is indicated. Otherwise, the event is only indicative of link bit errors.

In this category of tests, an XSTP-OFF session usually detects the loss by a STAT or a USTAT segment bringing back a non-empty gap report. The session responds 
by updating the next unacknowledged segment, which may expand the congestion window. Then, the session examines the gap report by applying some validation on each reportedly missing segment. In the case of a USTAT report, the segments should not have been retransmitted before. For a STAT report, on the other hand, enough time (usually one RTT subject to backoff) must have passed since each segment was last transmitted. After examining all the gaps, and should at least one segment need retransmission, the session performs the required retransmissions before closing its congestion window using the congestion avoidance algorithm. The session then continues its normal transmission of new data segments only if there is still room in its send window. Although the USTAT acknowledgment mechanism is somehow resilient to premature reporting of gaps due to packet reordering, both the USTAT and STAT mechanisms are not totally immune from reporting bogus gaps. This bogus reporting can occur in the case of unexpected levels of packet reordering in the network.

Similarly, when an XSTP-ON session receives a STAT or USTAT gap report, it performs the usual validation on the report. However, should the session determine that one or more segments pass the criteria and hence need retransmission, it does not perform the retransmission but rather activate the probing mechanism. This activation involves suspending the new data transmission and initiating a probing cycle. The cycle does not finish until a specific number (two in this case) of consecutive probe exchanges are completed. If the probing mechanism detects an increase in the 
RTT of the network compared to the RTT prevailing before the cycle got triggered, the mechanism takes this increase as a sign of congestion. The probing mechanism reacts by applying measures similar to those applied by XSTP-OFF. However, if no increase in the RTT is detected, the mechanism resumes the new data transmission with Immediate Recovery. Moreover, if any probe acknowledgment brings back an empty gap report, the probing mechanism considers that a sign of premature probing. The mechanism reacts by self--terminating and resuming transmission with Immediate Recovery.

\subsubsection{Effective Throughput}

In this category of tests, the experiments are performed with a mean duration of 1 second and 10 seconds, as shown in figures 6.1 and 6.2 . In each case, the tests are repeated with different error intensities ranging from 0 to $50 \%$. The effective throughput is measured in each case. One important observation is that XSTP-ON achieves consistently higher effective throughput than XSTP-OFF (from 3 to $150 \%$ ). This is due in large to the probing mechanism's ability to proceed with Immediate Recovery when link errors are detected, and therefore avoid to unnecessarily reduce the transmission rate. However, the advantage is more apparent in the 10 -second tests than in the 1-second tests. The reason relates to the insufficient time in the 1second tests for the sender to demonstrate its full error control capabilities. Sessions in both cases expand their congestion window size in the error-free phase. However, 


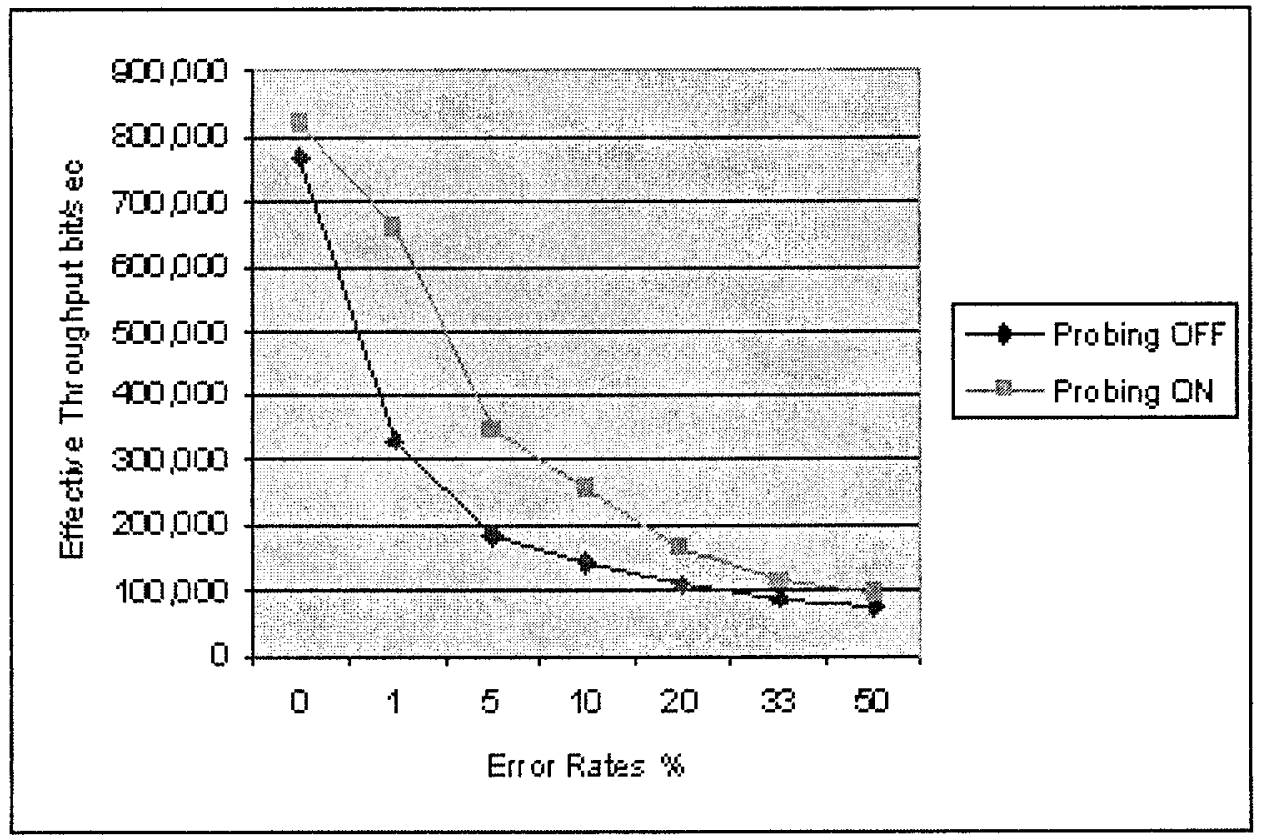

Figure 6.1: Effective throughput under bit corruption with 1 second mean duration

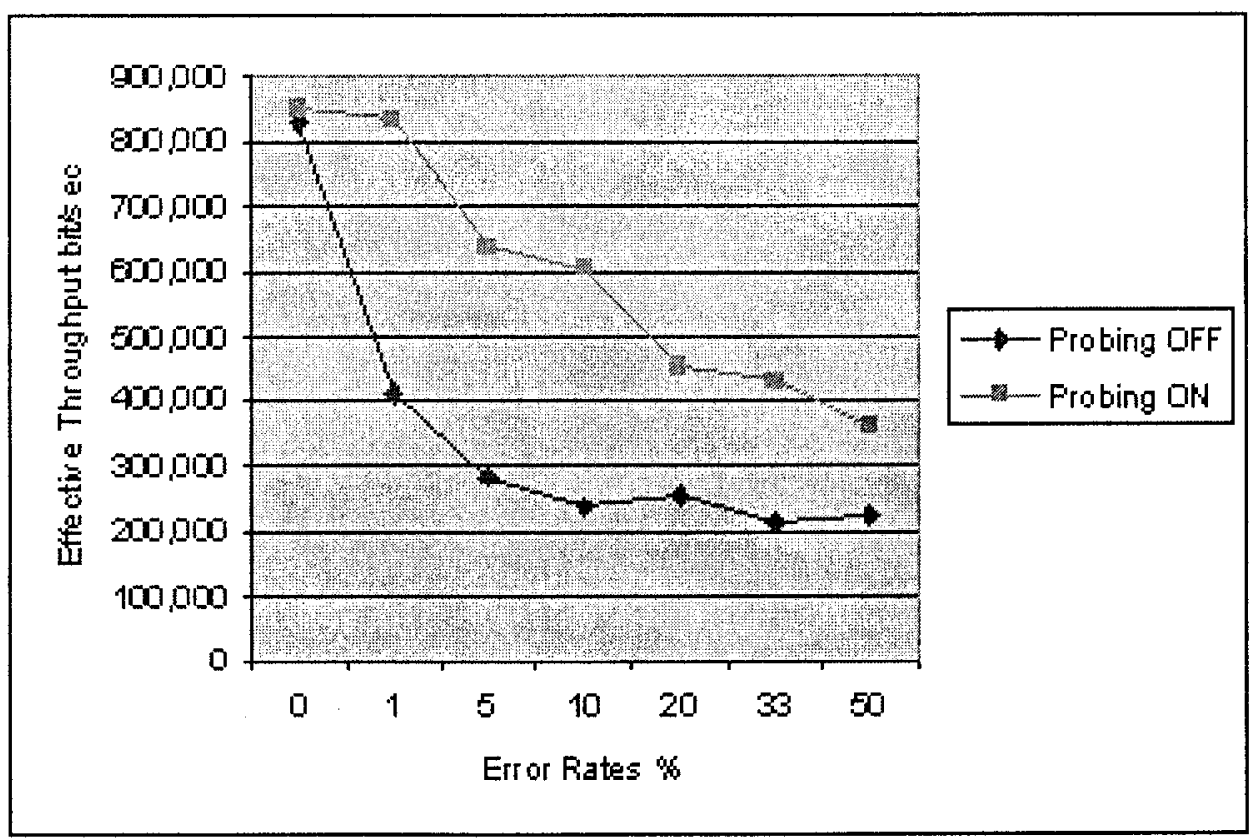

Figure 6.2: Effective throughput under bit corruption with 10 seconds mean duration 
that size reaches a more dramatic level under a 10-second phase than under a 1second one. In fact, the size after the 1 second cycle is usually not that different from the initial window size.

Another observation is that the more intense the error condition, the longer it takes the probing cycle to finish, due to the high probability of probe or acknowledgement loss. During that cycle, the session is effectively sitting out the error condition. Also, in case of severe error conditions with deteriorating RTTs that prevent an acknowledgment from coming back for sufficiently long time, the session can eventually go back to slow start after the probing is finished, which further stretches the connection time. This tends to make the session more conservative and therefore lowers throughput. On the other hand, if the error condition is light, the probing cycle finishes much more quickly. If no RTT extensions are detected, the session resumes transmission at the same speed as before probing.

It is also important to realize that the XSTP-OFF session does not close the congestion window further in response to more reported losses until the previous window of segments is confirmed received. This sequence has the beneficial effect of avoiding to close the congestion window multiple times in response to the same loss event. In addition, XSTP-OFF suspends its data transmission and only maintains a low frequency polling cycle until the window is reopened. This polling cycle becomes much 
like the XSTP-probing cycle, except with a different frequency that could also be subjected to backoffs in the absence of arriving STATs. Under severe error conditions, the polling cycle can be extended enough to miss windows of error-free transmission in the connection, which increases the overall connection time and hence lowers the achieved effective throughput. On the other hand, XSTP-probing maintains the probing frequency at one probe per RTT. The RTT value can still be updated with each arriving probe acknowledgment to approximate the RTT prevailing in the network. Also, since POLLs (probes) are being sent even before probing started, the chance to quickly collect the requested number of acknowledgments (STATs) improves considerably under moderate error conditions. The aforementioned generally favors effective throughput as transmission is resumed much quicker after appropriately adapting to the error condition.

\subsubsection{Overhead}

The total overhead is plotted against the various bit corruption rates in figures 6.3 and 6.4. The figures show that the probing mechanism generally reduces the total transmission overhead. As XSTP-probing activates the probing cycle, it suspends new data transmission to protect new packets from getting dropped. Through that probing cycle, the mechanism effectively waits until the error condition clears away before committing to new data transmission. In addition, the probing mechanism 


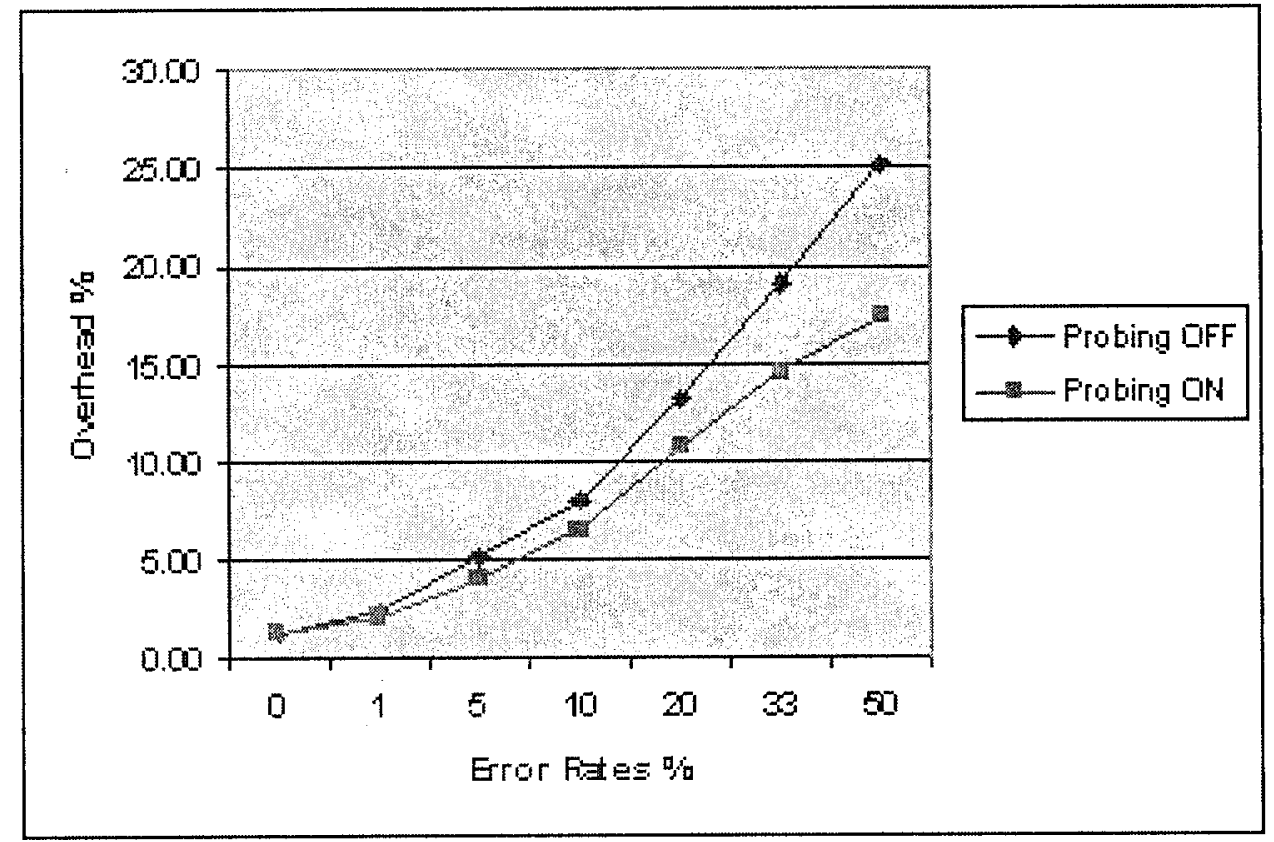

Figure 6.3: Overhead under bit corruption with 1 second mean duration

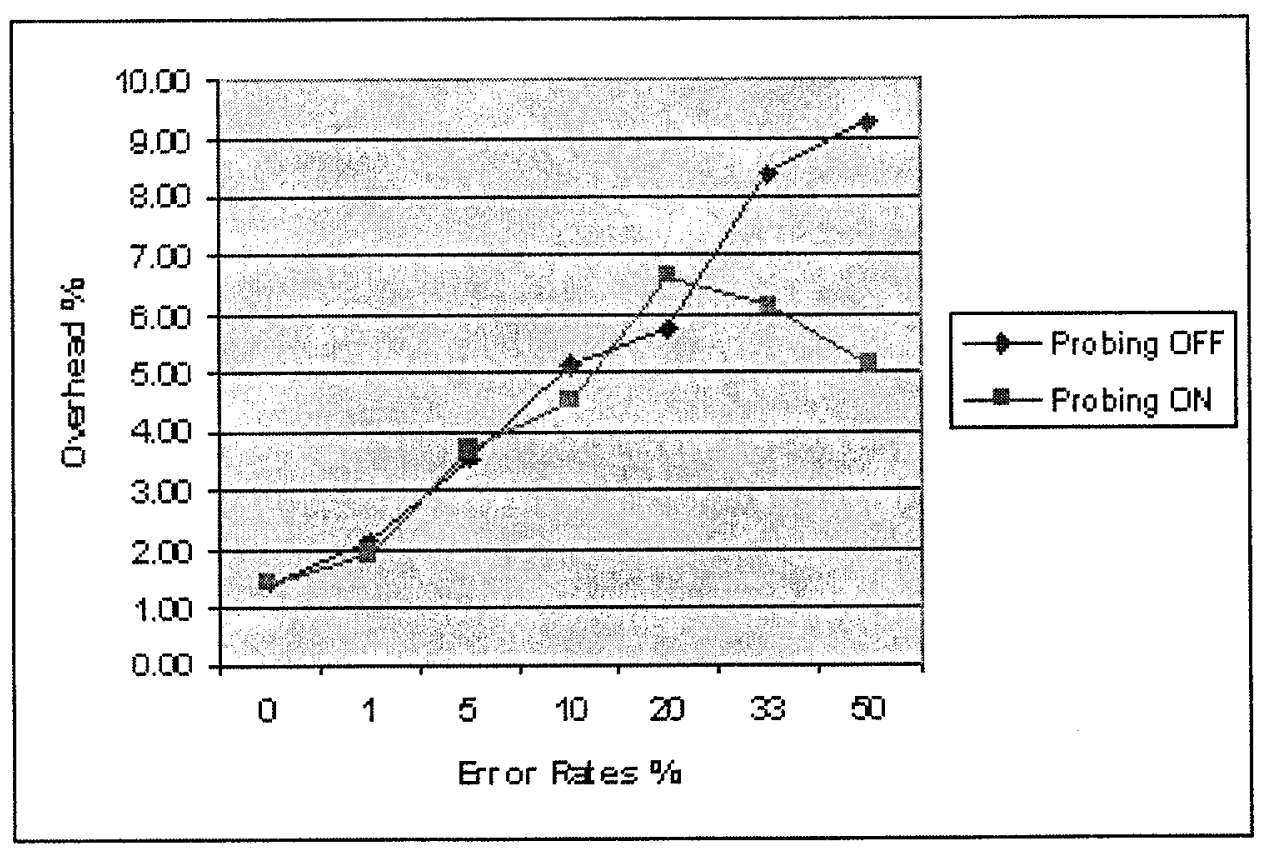

Figure 6.4: Overhead under bit corruption with 10 seconds mean duration 
tends to keep the session's send window at higher levels due to its Immediate Recovery option when link errors are detected. However, this also has the side effect of increasing the number of initial losses incurred by the session at the start of a loss event. At some error levels (like at $5 \%$ and $20 \%$ when the duration is 10 -second), the reduction in overhead achieved by suspending transmission is more than recouped by the overhead incurred as a result of having a large window at the start of an error phase. Another observation is that at some level of increasing error rates (20\% in this case), the probing mechanism manages to actually reduce the overhead. This is in large due to ability of the probing mechanism to start its cycle earlier using the early timeout feature. The probing cycle also gets extended because of the deteriorating error rates. For these reasons, the session becomes more conservative, which leads to an overall reduction in the retransmission overhead. One more observation is that the overhead levels are lower in the 10-second tests than in the 1-second tests. The reason for that observation relates to the extended error-free phases, in which the session grows its send window to much higher levels than it does in the 1-second tests, leaving less packets susceptible to drops and hence reducing the number of retransmissions. In most cases, the probing mechanism succeeds in lowering the overhead by a significant ratio ( $44 \%$ at the $50 \%$ point in 10 -second duration tests). 


\subsubsection{Throughput/Overhead Ratio}

The Throughput/overhead ratio is a metric to measure the amount of throughput achieved per one percent of overhead. In figures 6.5 and 6.6 , it is shown that this metric is inversely proportional to error rate. As error deteriorates, the amount of throughput achievable per a percentage of overhead decreases. The figures also show that XSTP-probing helps the session achieve more effective throughput with a lower level of overhead expenditure. This advantage increases as the mean duration increases from 1 to 10 -second, mainly due to the impressive gains in the effective throughput and the reduction in the overhead achieved in the 10-second tests.

\subsection{Handoff Tests}

In these tests, packets in both directions are dropped in the bad state with a $100 \%$ probability, marking a period of "blackout", where connectivity is temporarily lost. In the good state, packets are forwarded after being subjected to one of the two configured delays. If the error condition occurs with noticeable increases in $\mathrm{RT} T$, it is indicative of an intermediate router over-buffering, usually a symptom of a serious congestion condition in the network. The duration and frequency of that condition depends on the transport behavior of competing traffic in the network. However, if that period occurs without any RTT extensions, it is indicative of a handoff event. A handoff occurs due to the mobility of both the user terminal and the satellite. The 


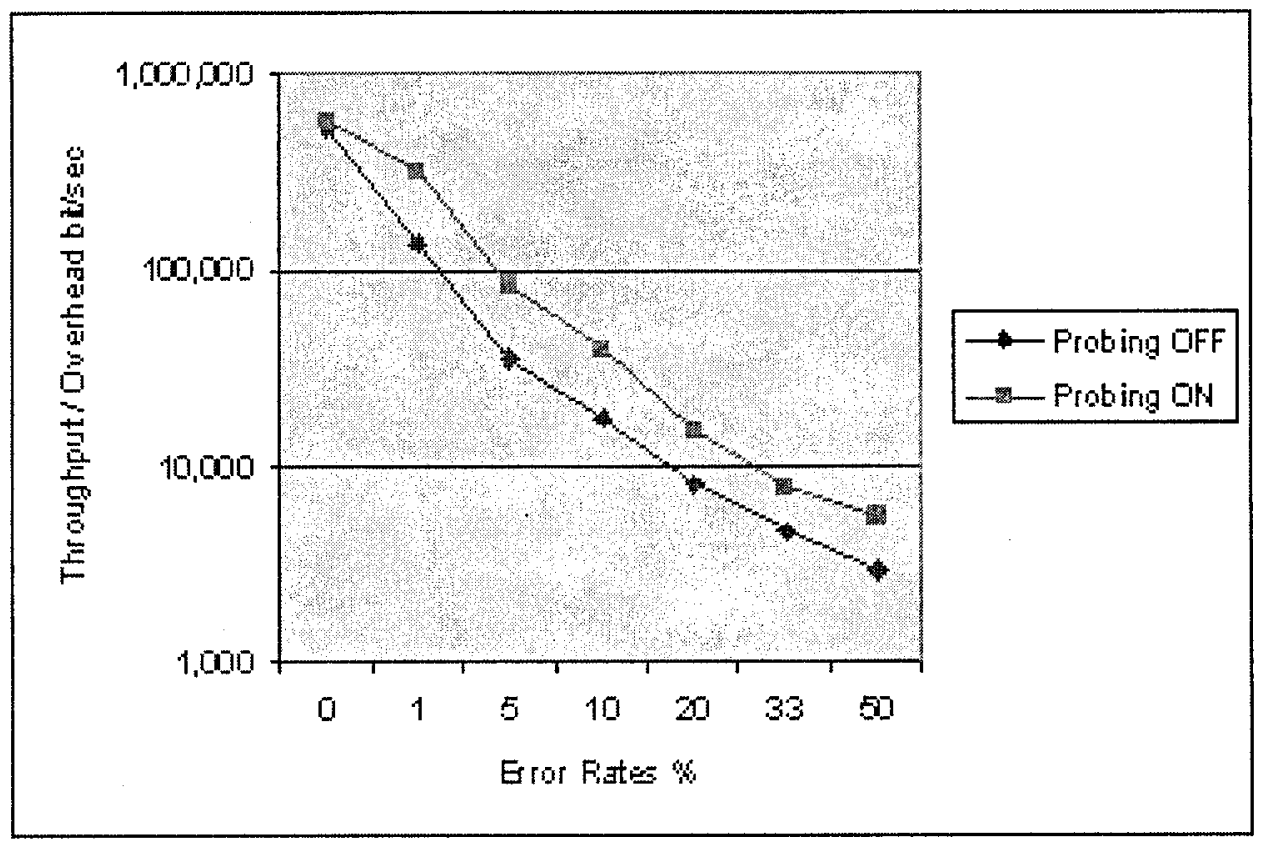

Figure 6.5: Throughput/overhead ratio under bit corruption with 1 second mean duration

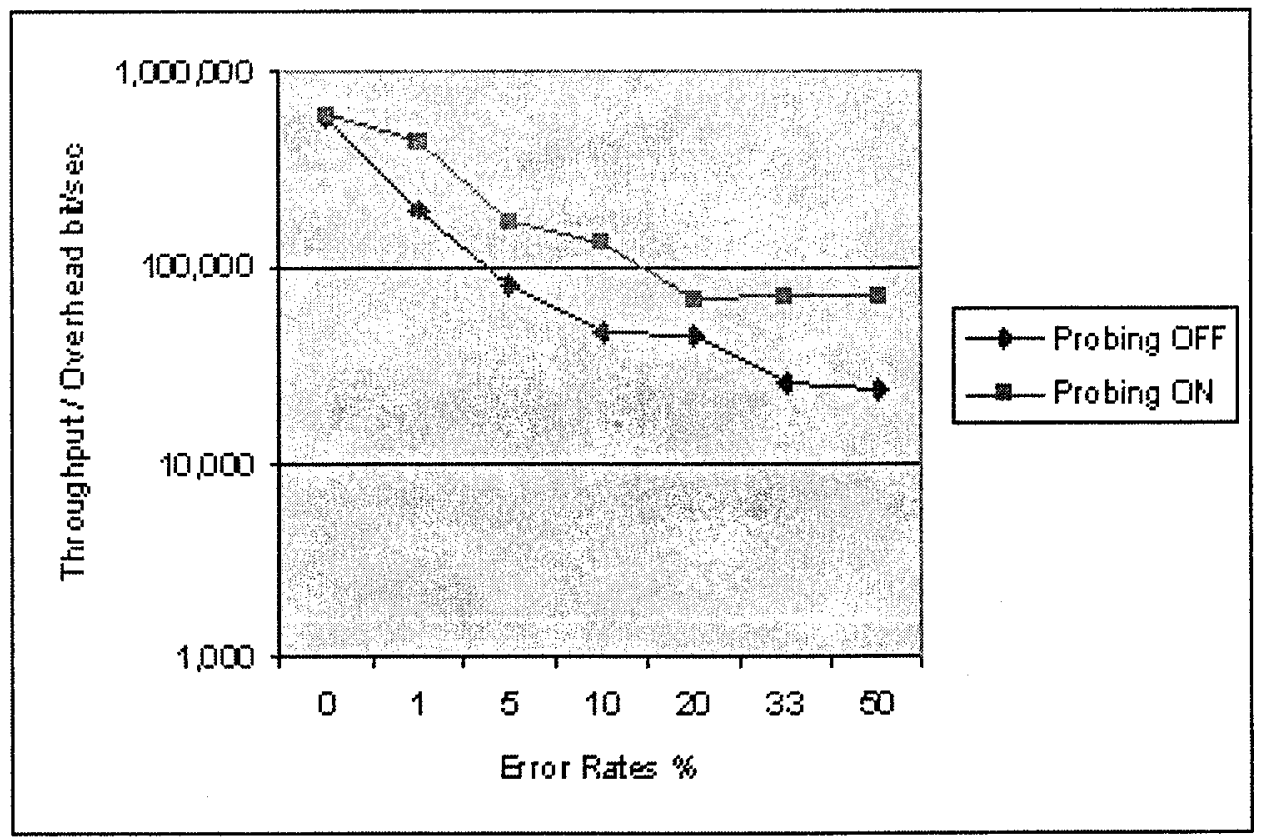

Figure 6.6: Throughput/overhead ratio under bit corruption with 10 seconds mean duration 
event takes place when either a satellite or a base-station is switched over. This period can vary in duration (rendezvous) or frequency depending on the speeds of both the satellite and the user terminal. In general for tests in this category, the connection is mainly in the good state and only frequently in the bad state.

An XSTP-OFF session does not detect the blackout period, hence the loss, until the period is over and either a STAT or a USTAT segment brings back a non-empty gap report in the following good state. This inability to sense the loss in connectivity makes the session susceptible to drain some or all of the segments in its send window. Also, as a result of applying congestion control measures, the session slows down its transmission speed before retransmitting the reportedly lost segments.

On the other hand, an XSTP-ON session can detect the blackout period much quicker by detecting a break in the POLL/STAT interleaf. This ability allows the session to only dump a fraction (1/POLLS_PER_RTT) of its current send window rather than the whole window, which translates into gains in both effective throughput: and energy efficiency. As soon as the session detects the loss, it activates the probing mechanism. In a LEO satellite environment, the RTT can moderately fluctuate with the mobility of the satellite, relative to the terminal user. The RTT can also increase due to a congestion condition starting to build up. In these cases, XSTP-ON can prematurely go into probing mode, as a result of the early-timeout feature. However, 
as soon as the presumedly lost STAT segment arrives containing an empty gap report, the probing mode is immediately terminated with virtually little if any loss in throughput.

\subsubsection{Effective Throughput}

In this category, test are performed with handoff rendezvous of 0.5 and 1 second, as shown in figures 6.7 and 6.8 . In each case, the tests are repeated with various handoff rates ranging from 1 to $15 \%$. The effective throughput is measured in each case. The first observation is that throughput is inversely proportional to the rate (hence the frequency) of handoff. The reason relates to each handoff occurrence costing the connection a portion of the send window worth of retransmissions, which increases the total connection time. Also, the throughput is expected to be inversely proportional to the rendezvous duration, due to the longer suspension of transmission. However, a comparison of the 0.5 and 1 second figures does not confirm this expectation. The reason for that relates to the rendezvous being relatively short when compared to the RTT, while the good phase duration is an order of magnitude larger than the RTT. For example, in the 1-second Figure, the rendezvous is double that of the other Figure, but the good phase durations are also double those in the other Figure. The loss in throughput realized by doubling the rendezvous is more than made up for by also doubling the good phase durations. This effect usually makes the connection obtain larger send windows and therefore shorter total connection time. 


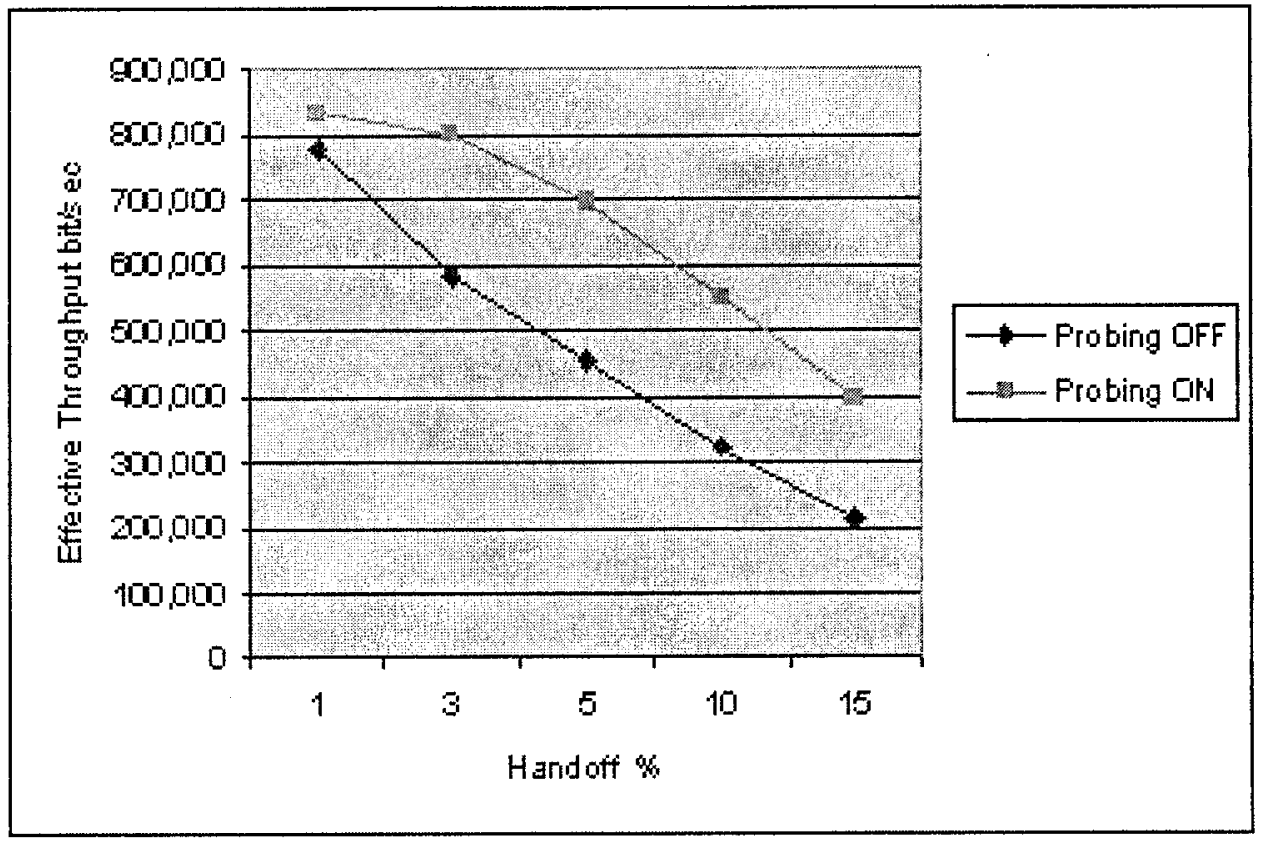

Figure 6.7: Effective throughput under handoff with 0.5 second rendezvous

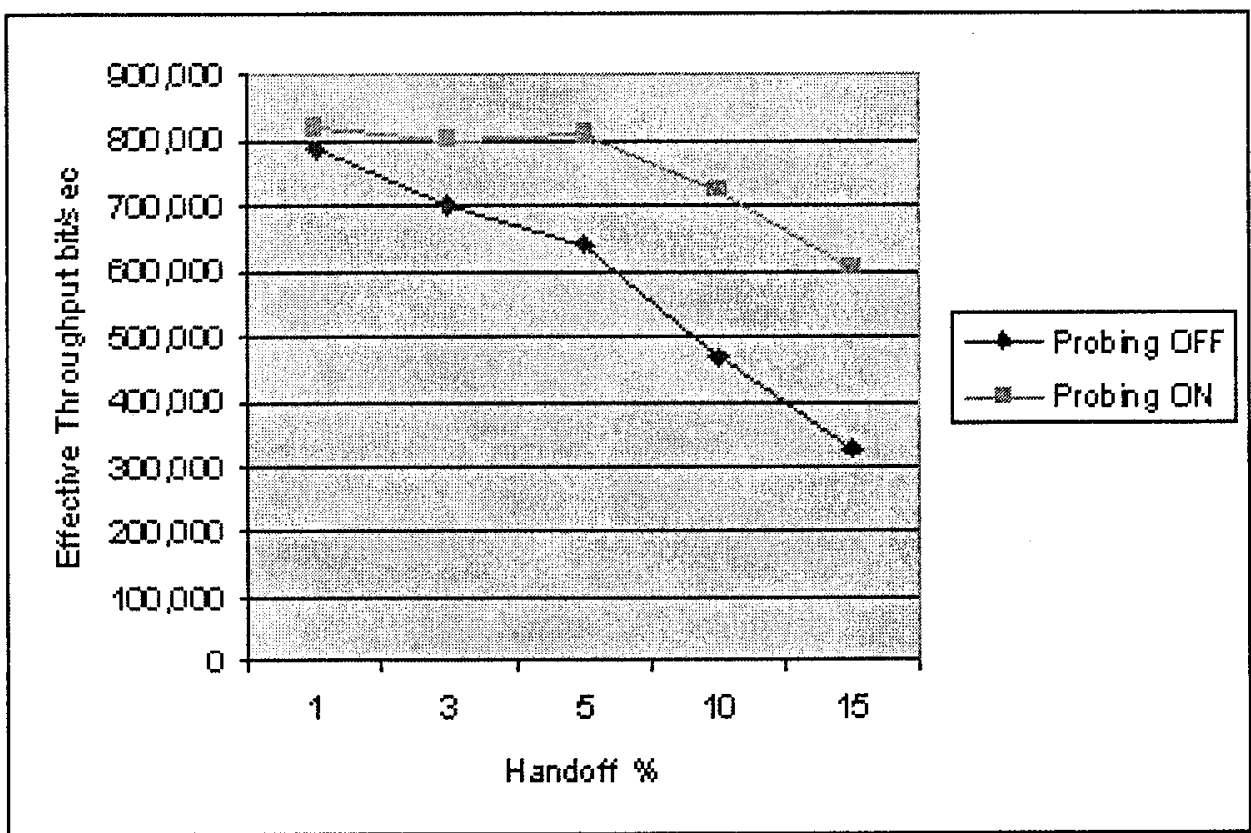

Figure 6.8: Effective throughput under handoff with 1 second rendezvous 
Another important observation is that XSTP-ON achieves consistently higher effective throughput than XSTP-OFF (from 4 to $86 \%$ gain). The main reason for that observation is the ability of XSTP-ON to differentiate congestion from handoff situations. This ability makes the session more conservative in case of deteriorating RTTs, and more aggressive otherwise by keeping a higher congestion window. Another reason for the observation is the ability of the protocol to quickly suspend transmission as soon as it detects a "blackout" period, and therefore avoid many retransmissions that usually extend the connection time. The advantage of the probing mechanism is more apparent in the 1 -second tests due to the longer good state duration, which results in a bigger send window when the session enters the handoff state. Therefore, more packets get dropped for the 1-second tests than for the other tests. Also, the number of packet drops saved by the probing mechanism translates directly into handsome gains for the connection effective throughput.

\subsubsection{Overhead}

The total overhead is plotted against the various handoff rates in figures 6.9 and 6.10 . The general observation is that the expended overhead is proportional to the handoff rates. This observation is mainly due to the increase in segment loss and hence the retransmission resulting from more frequent handoff events. The overhead is also expected to be proportional to the handoff rendezvous. However, a comparison of the overhead levels in the 0.5 and 1 second figures does not confirm this expectation. In 


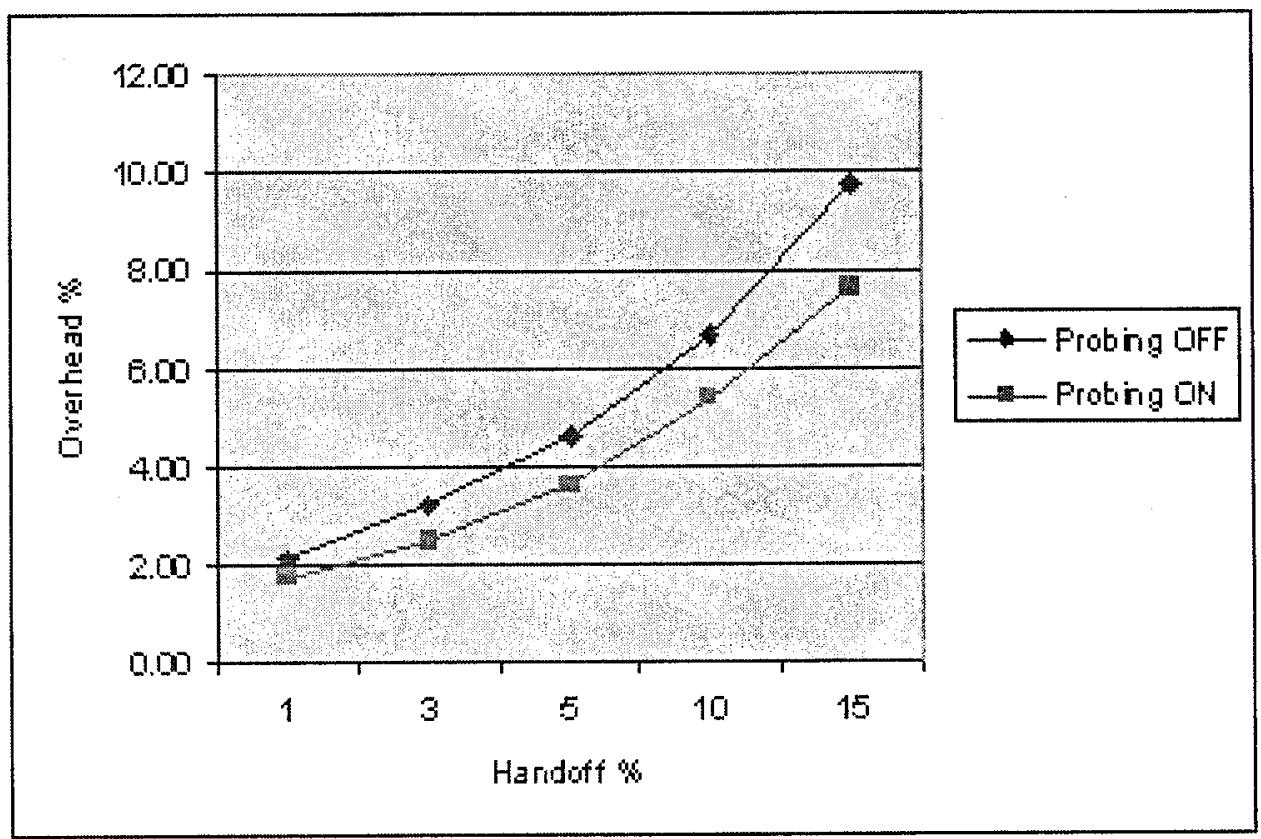

Figure 6.9: Overhead under handoff with 0.5 second rendezvous

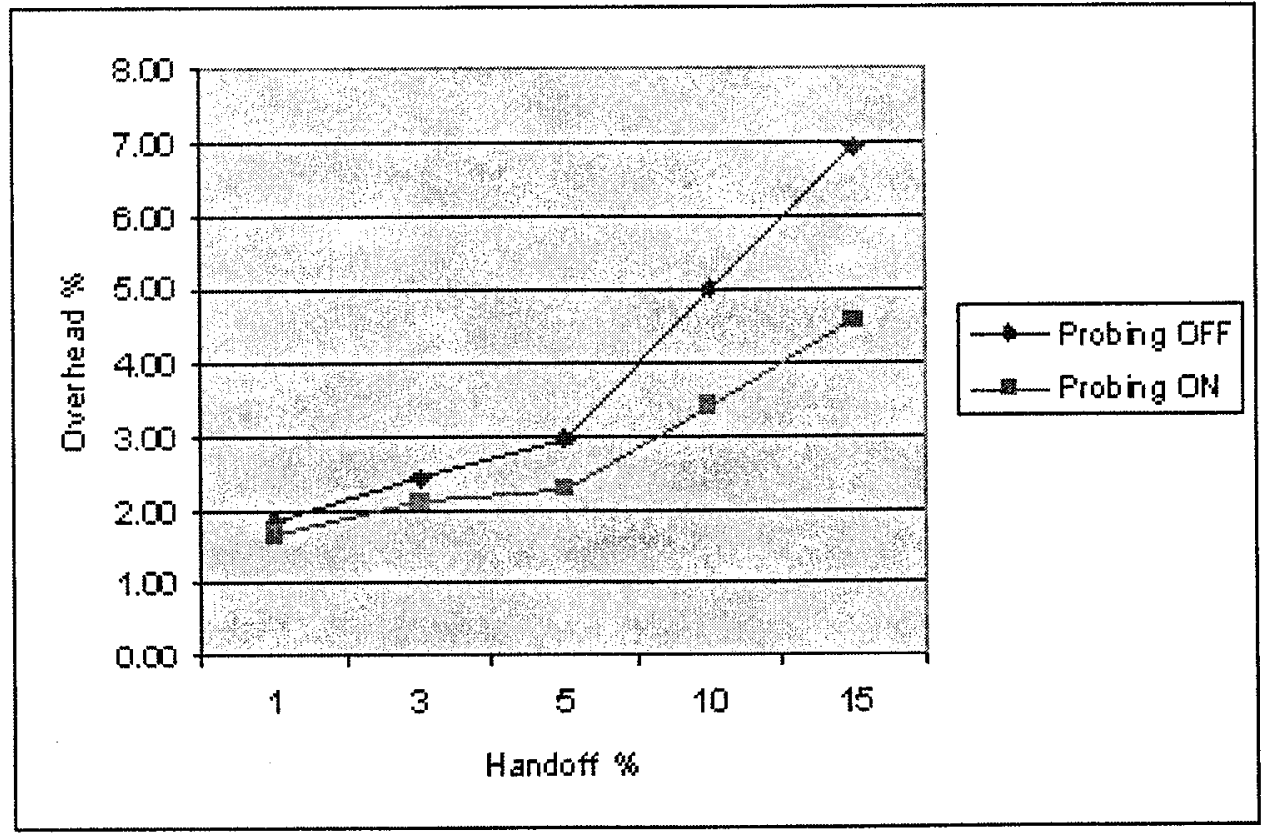

Figure 6.10: Overhead under handoff with 1 second rendezvous 
the 1-second Figure, the rendezvous is doubled but the good state duration is also doubled from that in the other Figure. The increase of overhead achieved by doubling the rendezvous is more than wiped out by the overhead savings resulting from faster transmission in the doubled good phase duration.

As mentioned, the XSTP-ON session suspends new data transmission as soon as it activates probing by the early-timeout event. That suspension effectively reduces the number of segments lost from the send window and therefore reduces the retransmission overhead. Also for the control overhead, XSTP-ON produces slightly less POLL and STAT traffic than does XSTP-OFF. This reduction is mainly due to the probing mechanism adjusting the polling (probing) rate to once per RTT while the mechanism is on. On the other hand, XSTP-OFF keeps its regular polling rate. It is interesting to realize that this much smaller polling rate does not noticeably affect overhead since the handof rendezvous is usually quite short. Generally, the probing mechanism succeds in lowering the overhead by a significant amount (34\% at the $50 \%$ point in 1 -second rendezvous tests).

\subsubsection{Throughput/Overhead Ratio}

In figures 6.11 and 6.12 , it is shown that the throughput/overhead ratio is inversely proportional to the handoff rate. Also for the same handoff rate, the efficiency is directly proportional to the handoff rendezvous, mainly because it is more proportional to the good state duration than it is inversely proportional to the rendezvous. The 


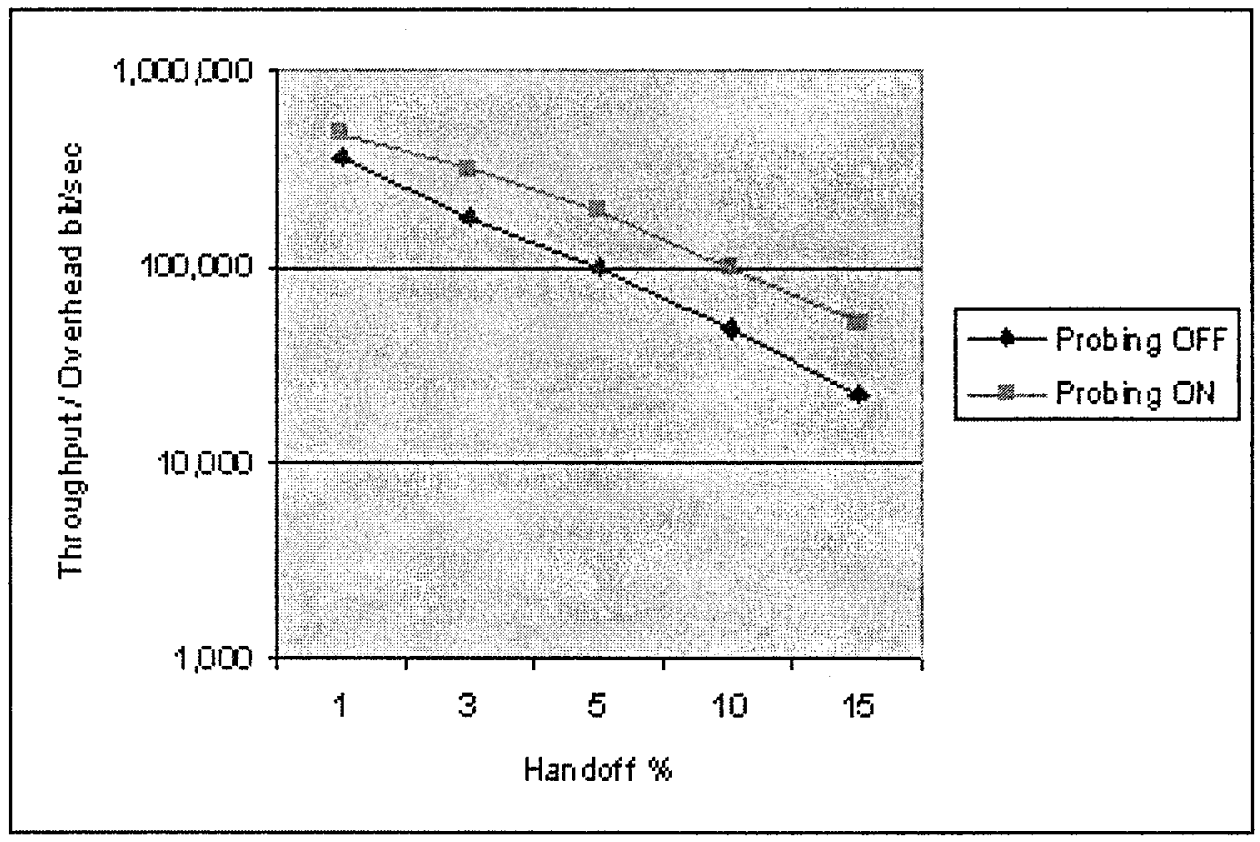

Figure 6.11: Throughput/overhead ratio under handoff with 0.5 second rendezvous

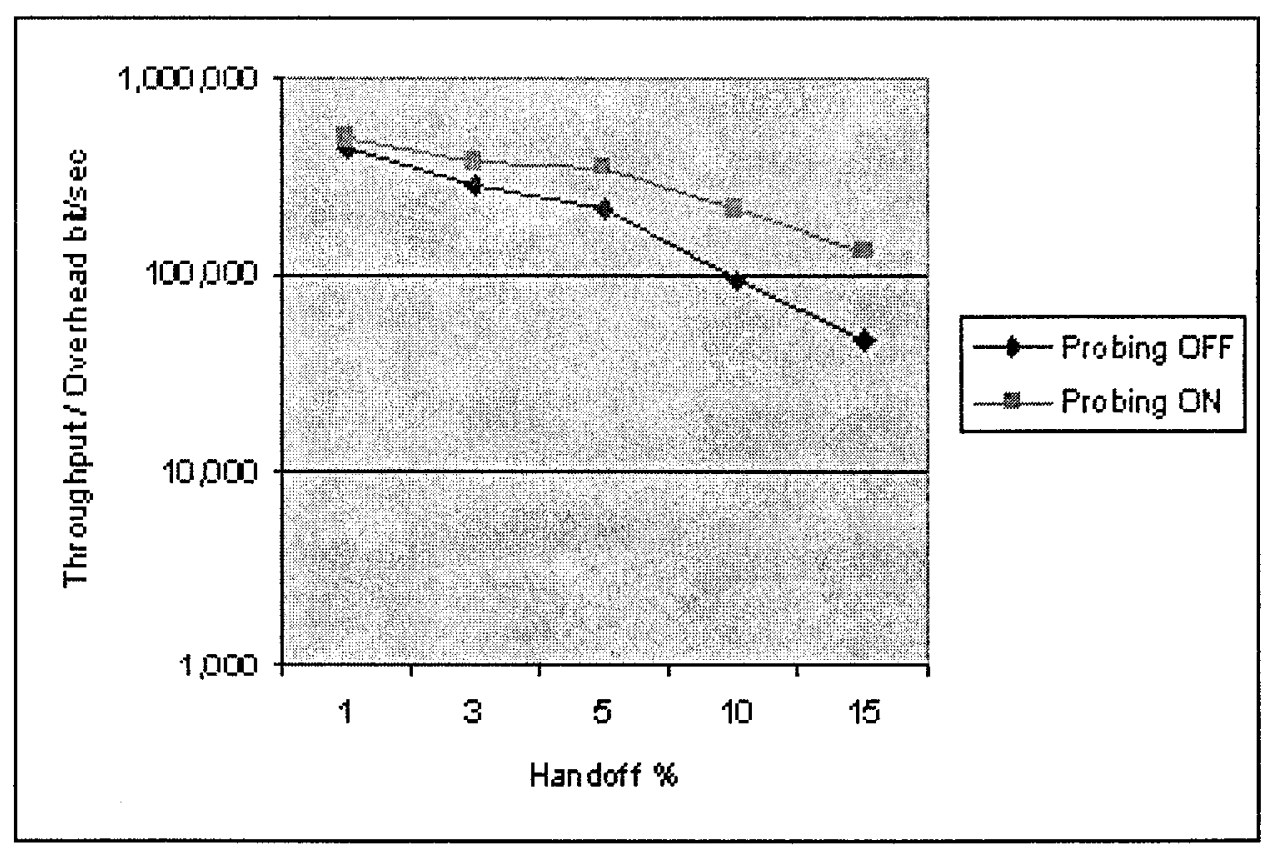

Figure 6.12: Throughput/Overhead ratio under handoff with 1 second rendezvous 
figures also show that using XSTP - probing helps the session achieve more throughput with the same level of overhead expenditure. The efficiency advantage increases from the 0.5 to the 1 -second tests, mainly due to the impressive gains in the throughput and the reduction in the overhead achieved in the 1-second tests.

\subsection{Limited Connectivity Tests}

In these tests, packets in both directions are dropped in the bad state with a $100 \%$ probability, marking a period of lost connectivity: In the good state, which is usually much smaller, packets are forwarded after being subjected to one of the two configured delays. This setup exemplifies limited connectivity conditions that result either from extended physical obstruction to the satellite signal, or from the unavailability of a satellite in range due to limitations in the coverage. This condition can also occur due to the constant mobility of the satellite and the frequent mobility of the terminal user. When the connection is later restored, it can either have a similar RTT to that before the interruption or a much deteriorated RTT that is usually indicative of congestion. Limited connectivity can vary in duration (rendezvous) or frequency depending on the satellite service coverage area and on the local environment (physical obstacles). In general for tests in this category, the connection is mainly in the bad state and only frequently in the good state.

An XSTP-OFF session does not detect the loss of connectivity, hence the segment 
loss, until it is over and either a STAT or a USTAT segment brings back a non-empty gap report in the good state. This inability to sense the loss in connectivity makes the session susceptible to drain some or all of the data segments from its send window. While connectivity is lost, the session maintains its usual polling cycles to sense when connectivity gets back. However, due to the extended connectivity loss periods, the polling cycles get extensively backed off to a level that compromises the ability of the session to detect the mainly short connectivity windows. In this case, the session either misses one or more connectivity windows or at best detects a window long after one started. As soon as the session detects the connectivity loss, it slows down its transmission before retransmitting the reportedly missing segments.

On the other hand, the XSTP-ON session can detect the connectivity loss period much quicker by detecting a break in the POLL/STAT interleaf. This detection ability makes the session only dump a fraction (1/POLLS_PER_RTT) of its current send window rather than the whole window, which translates into gains in both throughput and energy efficiency. As soon as the session detects the loss, it activates the probing mechanism. The mechanism maintains a probe-per-RTT cycle without applying any backoff. This feature has the advantage of quickly detecting the connectivity windows within one RTT. On the other hand, it can also have an adverse effect on the control overhead due to the amount of POLL traffic transmitted during the extended probing cycles. In the case of a premature timeout- event (a false alarm), the probing mode 
is terminated as soon as the next STAT arrives holding an empty gap report.

\subsubsection{Effective Throughput}

In this category, tests are performed with connectivity rendezvous of 5 and 10 seconds, as shown in figures 6.13 and 6.14 . In each case, the tests are repeated with various connectivity rates ranging from 1 to $30 \%$. The effective throughput is measured in each case. The first observation is that the more frequent the connectivity, the larger the chance of transmitting new data packets, and the shorter the overall connection time. Effective throughput is also proportional to the rendezvous of the connectivity, especially when the rendezvous is orders of magnitude larger than the RTT. The reason relates to the fact that during longer connectivity periods, the congestion window grows much bigger than during shorter periods, resulting in better throughput, hence shorter connection time.

Another important observation is that XSTP-ON achieves consistently higher throughput than XSTP-OFF (from 13 to $77 \%$ gain). The main reason relates to the ability of XSTP-ON to differentiate congestion from connectivity loss situations. This ability makes the session more conservative in case of deteriorating RTTs, and more aggressive otherwise by maintaining a higher congestion window. Another reason for this observation is the ability of the protocol to quickly suspend transmission as soon as it detects a loss in connectivity, and therefore avoid many retransmissions that usually extend the connection time. 


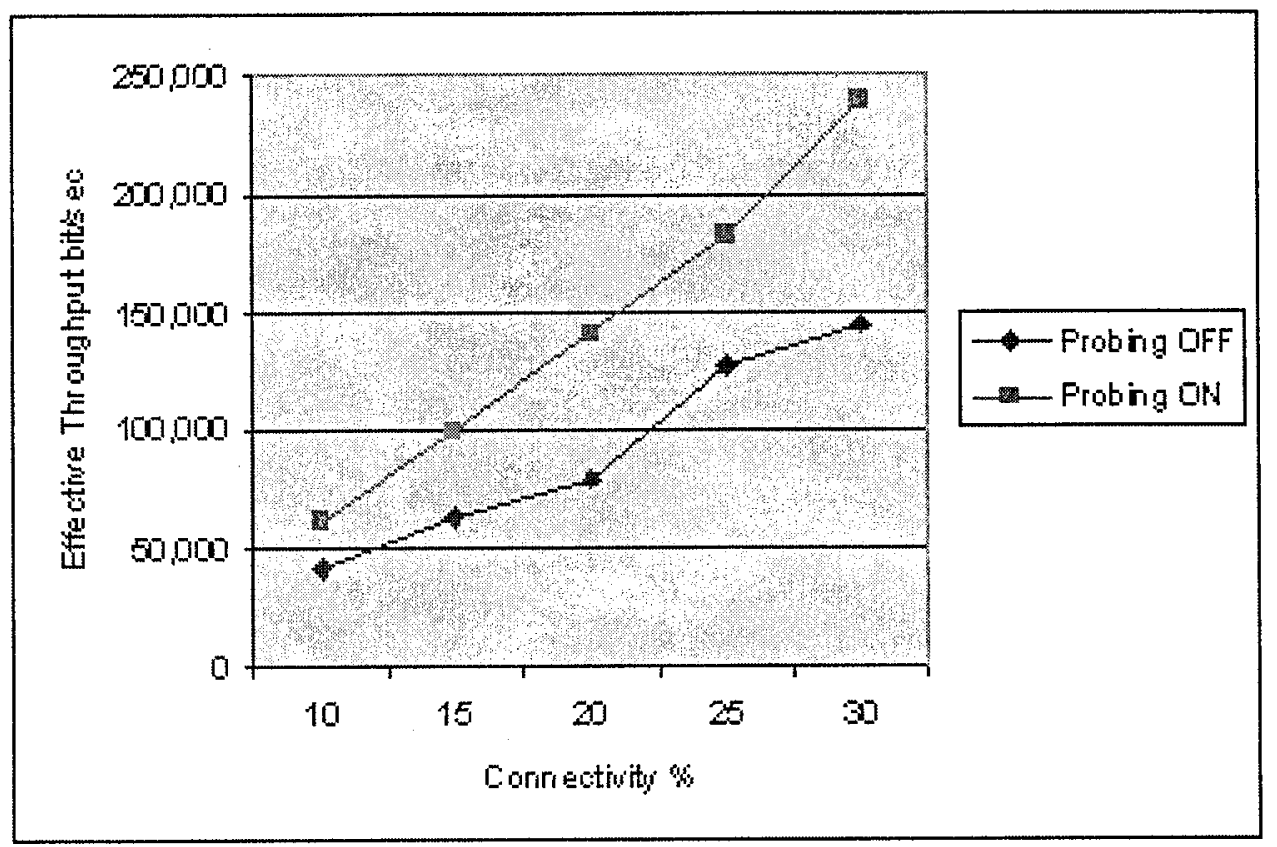

Figure 6.13: Effective throughput under limited connectivity with 5 seconds rendezvous

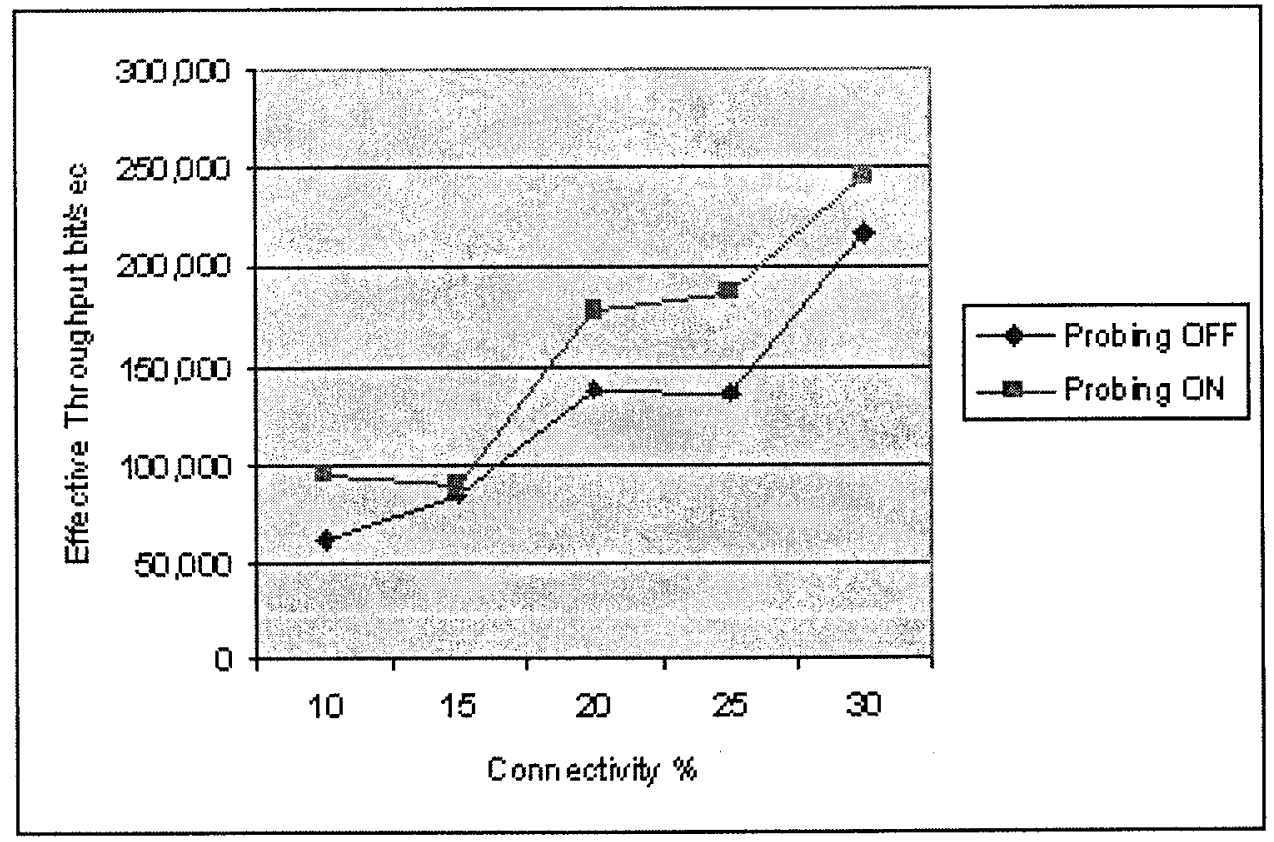

Figure 6.14: Effective throughput under limited connectivity with 10 seconds rendezvous 
Contrary to the expected, the probing mechanism appears to have a better advantage in the 5--second Figure than in the 10-second Figure. In the 10-second tests, the connectivity rendezvous is bigger but the loss of connectivity is also bigger. In fact, the loss of connectivity gets extended beyond XSTP's configurable maximum idle time. This situation forces XSTP-OFF to go back to slow start as soon as it detects the connection back. Since slow start allows exponential window growth, the connection catches up with throughput more quickly than in the 5-second tests. For this reason, the advantage of the probing mechanism to the session is lowered, but is not canceled. The probing mechanism still saves the session multiple round trips by immediately restoring the old throughput level when congestion is not detected.

\subsubsection{Overhead}

The total overhead is plotted against the various connectivity rates in figures 6.15 and 6.16. The general observation is that the expended overhead is not changing much in response to the changing connectivity rates. The reason for that observation becomes apparent when a closer look at the overhead components is taken. The major component is the retransmission overhead, which is usually incurred with every connectivity loss event. The value of that overhead is roughly the product of the number of connectivity loss events and the average number of drops per event. In the tests, the bigger the connectivity rate, the higher the average send window the session maintains. That higher window translates into more average drops in the bad 


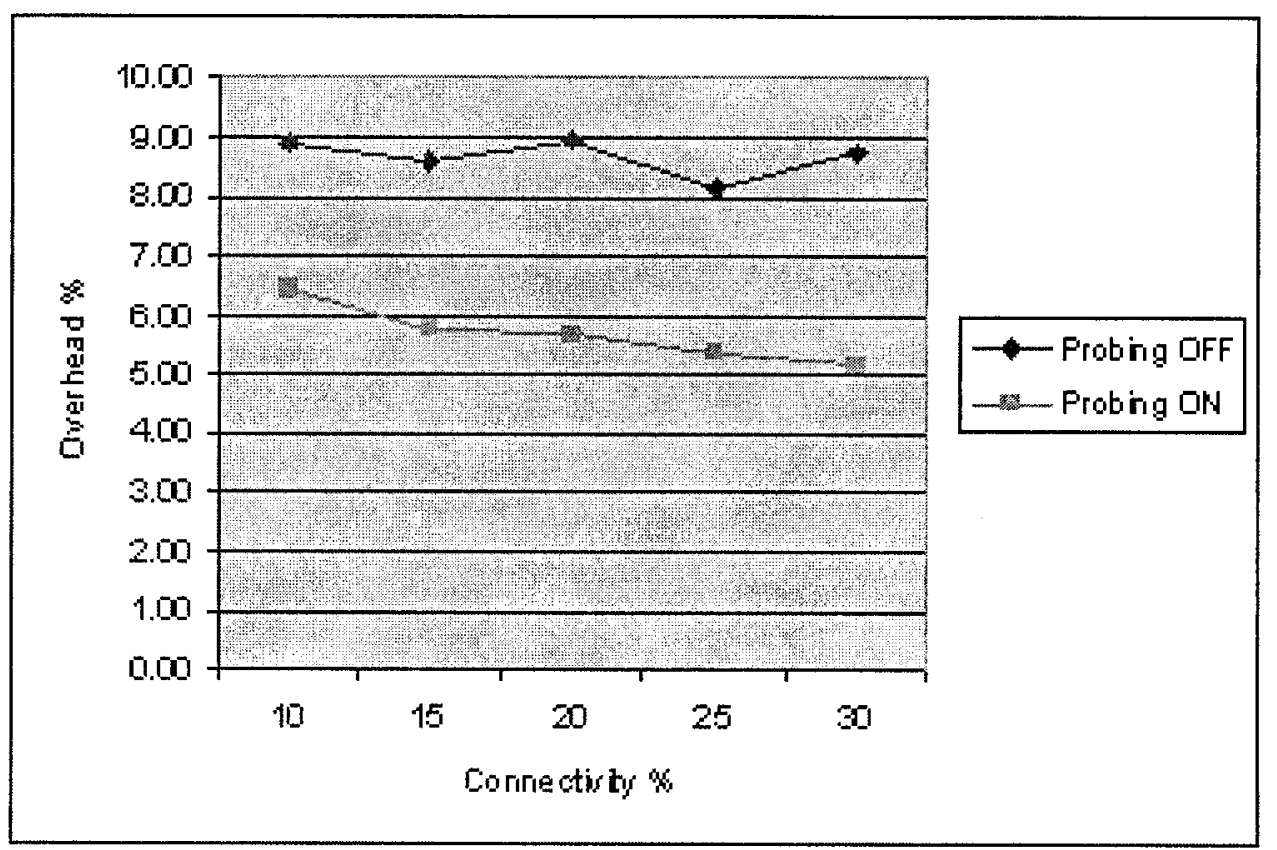

Figure 6.15: Overhead under limited connectivity with 5 seconds rendezvous

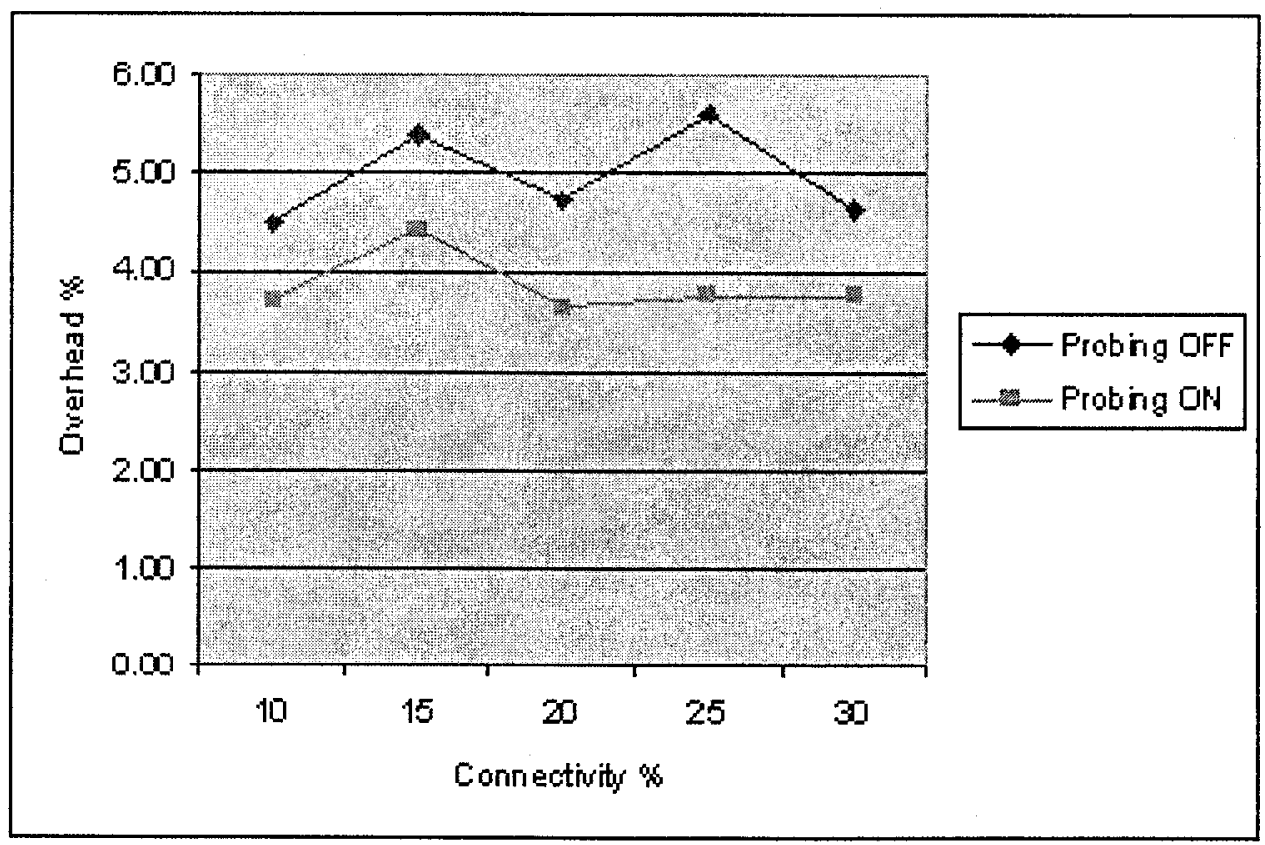

Figure 6.16: Overhead under limited connectivity with 10 seconds rendezvous 
state. At the same time, the higher window also means that more packets on average will make it through in the good state, leading to a shorter connection time and therefore a lower number of connectivity loss events. In short, one part of the product increases while the other decreases. Therefore, the total retransmission overhead is kept somewhat in a tight range.

Similarly, an explanation can be offered why the 10-second tests incur less overhead than the 5-second ones. The reason is obviously due to the larger connectivity period, in which more packets are forwarded, leaving less packets exposed to the connectivity loss events. Also taking a closer look at the control overhead, it is found that in the 10-second tests, the connectivity loss events get more extended, forcing the polling cycles to be more backed off. This backoff reduces the amount of POLL traffic, giving another reason for the reduced total overhead.

In addition, it is also shown that XSTP-ON sessions incur less overhead than XSTP-OFF sessions. The reason relates to the ability of the probing mechanism to detect the connectivity loss event much quicker using its early-timeout heuristic. That ability reduces the amount of segments dropped per loss event. Also by adapting to the error conditions in the network, the probing mechanism maintains a higher level of throughput and therefore a shorter connection time. This feature allows the connections to experience fewer loss events in total. It is also interesting to notice that the probing mechanism's advantage is lower in the 10-second tests than in the other 
tests. This lower advantage is mainly due to XSTP-OFF going back to slow start when connectivity is resumed. In this case, $\mathrm{XSTP}-\mathrm{OFF}$ maintains a higher average throughput, which shortens the total connection time. Again, a shorter connection experiences fewer connectivity loss events and hence has less retransmission overhead. Another reason is the extension of the probing cycle in the longer connectivity loss events. This extension leads to an increase in the control overhead incurred by the probing mechanism (a probe per RTT), which lowers the overall advantage of the probing mechanism for these tests.

\subsubsection{Throughput/Overhead Ratio}

In figures 6.17 and 6.18 , it is shown that, throughput/overhead ratio is mainly proportional to the connectivity rate. Also for the same connectivity rate, the efficiency is proportional to the connectivity rendezvous. The figures also show that using XSTPprobing helps the session achieve more throughput with the same level of overhead expenditure. However, the probing mechanism's advantage decreases from the 5second to the 10-second tests, mainly due to the reduced gains in the throughput and energy efficiency made in the 10-second tests. 


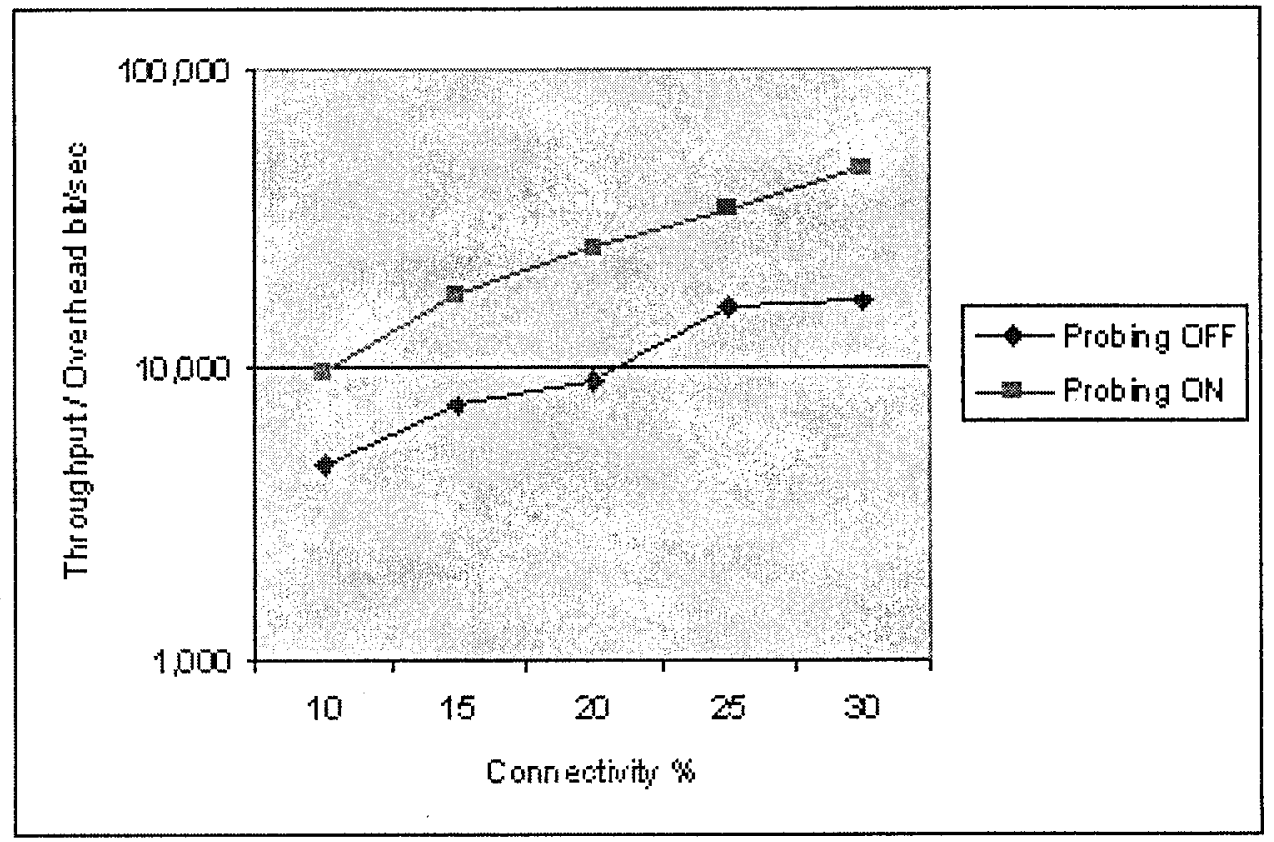

Figure 6.17: Throughput/overhead ratio under limited connectivity with 5 seconds rendezvous

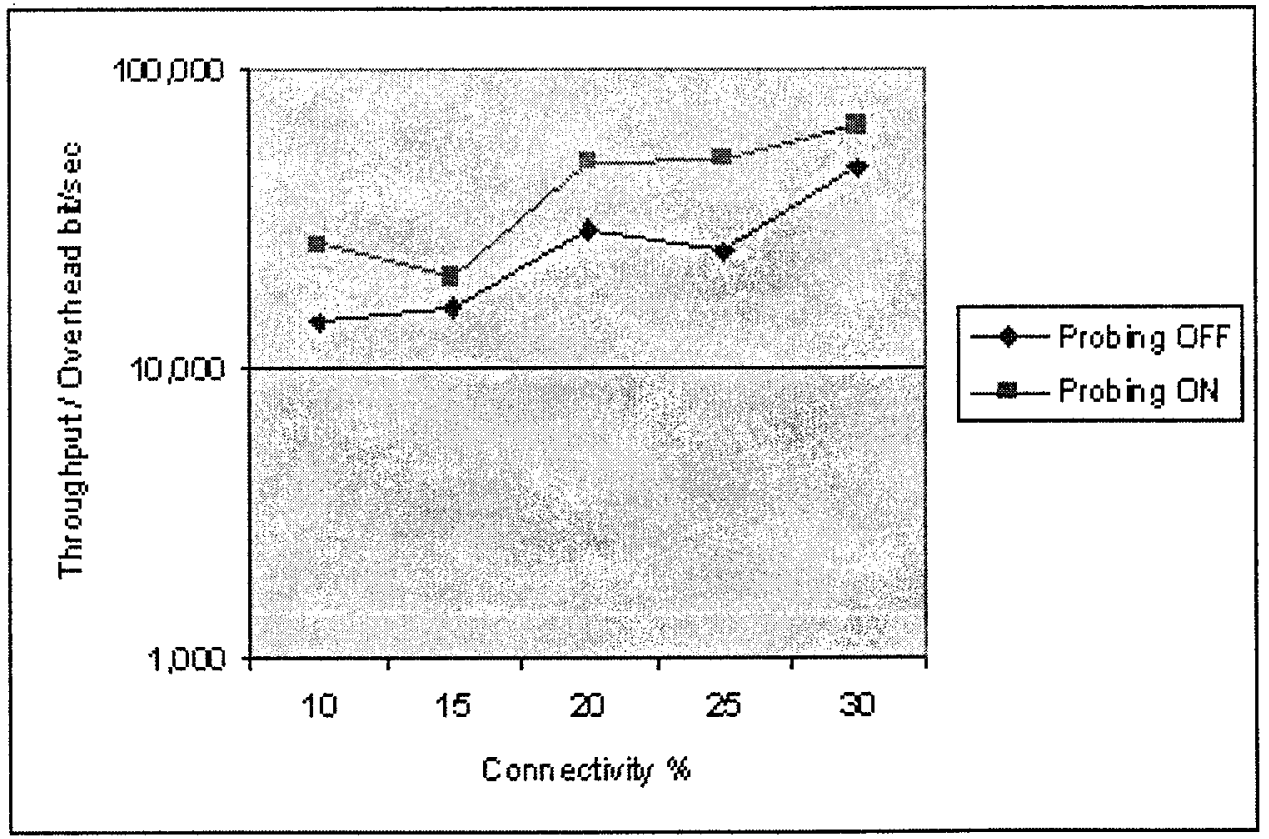

Figure 6.18: Throughput/overhead ratio under limited connectivity with 10 seconds rendezvous 


\section{Chapter 7}

\section{Conclusion and Future Work}

LEO satellite access networks present a challenge for an efficient and reliable transport of data. The main reason is attributed to the failure of standard transport protocols to deal with the diverse characteristics of heterogeneous links including LEO satellite links. Specifically, these protocols are congestion-centric and they lack an error control strategy that is adaptive to various error conditions in the network. The Satellite Transport Protocol (STP) is a recent proposal that addresses many issues with transporting data over satellite links. However, STP inherits the same congestion control bias that exists in its ancestor protocols.

In this thesis, a new error control strategy for STP that adapts to the available error condition in the network is presented. The strategy is based on an end-to-end probing mechanism installed at the STP sender and activated when a segment loss is detected. A loss is detected by either an early timeout event or an explicit feedback from the STP receiver. The idea of the probing mechanism is to suspend new data 
transmission upon discovering a loss and wait until the error situation improves before adjusting the transmission rate. The mechanism compares the connection's RTT both before and after probing, to determine if the error condition is congestion or linkrelated. After this comparison, the mechanism retransmits the lost segments and resumes transmission of new data.

To test the new probing mechanism, the STP protocol is implemented in the PIX framework. PIX offers the new protocol an object-oriented design, an established pattern with clearly defined interfaces, ease of configuration and deployment and finally access to light-weight libraries. The new probing mechanism is also implemented as a configurable option in the protocol. The new protocol with the probing option is called the eXtended Satellite Transport Protocol (XSTP).

The tests performed rely on comparing the performance of XSTP with the probing option turned on and off. These tests are performed in a simulated LEO satellite access network environment. For that simulation, two more PIX protocols are implemented: a link layer protocol based on the POSIX message queues; and an error generating protocol capable of producing different congestion and link error phases. The simulation setup involves three nodes: a data source node; a router node; and a data sink node. Only one bulk data transfer connection is run, from the data source through the router to the data sink. Three categories of tests are also defined: bit corruption tests; handoff tests; and limited connectivity tests. For each category, phases 
of network congestion are also generated. The measurements collected from each test include the total connection time and the various incurred overhead including: the retransmission overhead; the forward control overhead; and the reverse control overhead. In each case, three metric values are calculated: the effective throughput; the total transmission overhead; and their ratio.

The probing mechanism basically works by investing some transmission overhead and energy to investigate the nature of the error condition before adjusting the transmission rate. However, this overhead is more than recovered in the form of substantial throughput gains and energy savings. The test results confirm expectations that under intensive error conditions, a more conservative mode helps throughput, while under light or random error conditions, an aggressive bias is more appropriate. These results also proved to be more useful than either directly invoking congestion control and degrading the throughput for no reason, or sending more data segments that are likely to be dropped. In all these tests, the probing mechanism consistently helps the protocol achieve higher levels of throughput/overhead ratio mainly by increasing the connection effective throughput while maintaining lower levels of overhead.

An important point of note is that the probing mechanism is most helpful in mixed link and congestion error conditions, especially when link errors are more prevalent. However, the more the errors turn out to be congestion-related, the more conservative the connection becomes on average. This effect can counter the throughput gains 
achieved by the probing mechanism for non-congestion errors. At some point, the aggregate negative impact on throughput can balance and sometimes exceed the positive impact. The same situation can occur to overhead as well. It is prudent to leave the investigation of that pivotal point to a future work.

Furthermore, in each of the tests a particular kind of link error with some fixed parameters (duration and frequency) is simulated, to evaluate the effect of the probing mechanism in each case. However, a real network is modelled with a pattern of varying configurations (kind, duration and frequency) of the error model. While no claim is made that these results hold in general, but rather are indicative of the kind of gains attainable by using the probing mechanism, it will be interesting to see how the mechanism scales under varying configurations of the error model. It also helps if the probing mechanism can be tested in a real satellite access network.

There are many other points that could not be accommodated within the time frame of this thesis, that are worth investigating in a future work. One of these points is a more sophisticated decision-making criteria at the end of the probing cycle that possibly makes use of the following: history of previous decisions; the patterns of probing exchanges; and some other connection statistics. In addition, it also makes sense to investigate the probing mechanism under different traffic patterns, including HTTP, transactional and full duplex traffic. The mechanism can also be tested with longer RTT connections (available in GEO satellite paths). Another point 
to consider, is the level of energy expended during probing and whether this energy can be measured in different ways; other than merely the transmission overhead percentage.

It also makes sense to compare the XSTP-probing mechanism with TCP-probing. However, this comparison cannot be done by implementing TCP-probing in XSTP due to lack of sufficient assumptions like the availability of the ACK timeout concept in XSTP. Alternatively, the TCP protocol can be implemented in the PIX framework along with its probing mechanism. Both protocols can then be configured with a similar set of features and parameters and compared together. The comparison can show which mechanism is more efficient in adapting to the unique error characteristics of the underlying network. Another option is to implement the XSTP mechanism in TCP and compare it to other suggested error control strategies for TCP.

Furthermore, other possible topics for future work include modelling of the variation of delays of LEO satellites using a model of orbit, experimenting with different network topologies including inter-satellite links or limited bandwidth links. The probing mechanism is also worth investigating in the wireless domain or any other similar domain characterized by heterogeneous errors.

The research in the area of LEO satellite networks is still in its infancy. Specifically, transport protocols for LEO satellite networks are still far from being perfected. 
Many researchers and organizations are putting a concerted effort to enhance the performance of transport protocols in space. Very recently, NASA launched its SpaceDev CHIPSat program [RJS02]. The project is the first mission to use TCP/IP on an end-to--end satellite operation. The project aims to use robust Internet protocols to communicate in LEO satellite specific missions. Another attempt is made by a company called Teledesic [Koh96] to launch a global broadband, wireless Internet service using a constellation of LEO satellites covering the globe. These proposals and others help define the characteristics of the new generation of Internet connectivity. They also give perspective to future much appreciated efforts to enhance our quality of life. 


\section{Bibliography}

$\left[\mathrm{ADG}^{+} 00\right]$ M. Allman, S. Dawkins, D. Glover, J. Griner, D. Tran, T. Henderson, J. Heidemann, J. Touch, H. Kruse, S. Ostermann, K. Scott, and J. Semke. Ongoing TCP research related to satellites. RFC 2760, February 2000.

[AGS99] M. Allman, D. Glover, and L. Sanchez. Enhancing TCP over satellite channels using standard mechanisms. RFC 2488, January 1999.

[All98] M. Allman. On the generation and use of TCP acknowledgements. ACM Computer Communications Review, 1998.

[APS99] M. Allman, V. Paxson, and W. Stevens. TCP congestion control. RFC 2581, April 1999.

[Bar02a] M. Barbeau. Protocol implementation framework for linux (PIX). Technical report, 2002.

[Bar02b] M. Barbeau. Small satellite experiment. http://www.scs.carleton.ca/ barbeau, 2002.

[BB02] M. Barbeau and F. Bordeleau. A protocol stack development tool using generative programming. In Proceedings of Generative Programming and Component Engineering (GPCE), 2002. Lecture Notes in Computer Science 2487.

$\left[\mathrm{BKG}^{+} 01\right]$ J. Border, M. Kojo, J. Griner, G. Montenegro, and Z. Shelby. Performance enhancing proxies. RFC 3135, June 2001. 
[BKPV96] B. Bakshi, N. Krishna, D. Padhan, and N. Vaidya. A comparison of mechanism for improving TCP performance over wireless links. In Proceedings of ACM SIGCOMM' '96. Stanford, CA, August 1996.

[BPK97] H. Balakrishnan, V. Padmanabhan, and R. Katz. The effects of asymmetry on TCP performance. In Proceedings of Mobile Computing and Networking '97, pages 77-89, 1997.

[BPSK96] H. Balakrishnan, V. Padmanabhan, S. Seshan, and R. Katz. A comparison of mechanisms for improving TCP performance over wireless links. In Proceedings of ACM SIGCOMM '96, Stanford, CA, August 1996.

[Bra89] R. Braden. Requirements for internet hosts - communication layers. RFC 1122, October 1989.

[BSK95] H. Balakrishnan, S. Seshan, and R. Katz. Improving reliable transport and handoff performance in cellular wireless networks. ACM Wireless Networks, 1(4), 1995.

[BV98] S. Biaz and N. Vaidya. Sender-based heuristic for distinguishing congestion losses from wireless losses. Technical report, Texas A\&M University, June 1998. Tech. Rep. TR98-013.

[BV99] S. Biaz and N. Vaidya. Discriminating congestion losses from wireless losses using inter-arrival times at the receiver. IEEE Symposium $A S$ SET'99, Richardson, TX, USA, 1999.

[CCV02] S. Cen, P. Cosman, and G. Voelker. End-to-end differentiation of congestion and wireless losses. In Proceedings of ACM Multimedia Computing and Networking, volume 4673, pages 1-15, January 2002.

[CE95] M. Corson and A. Ephremides. A distributed routing algorithm for mobile wireless networks. ACM-Baltzer Journal of Wireless Networks, 1(1):6181, 1995. 
[FH99] S. Floyd and T. Henderson. The newreno modification to TCP's fast recovery algorithm. RFC 2582, April 1999.

[Gur00] A. Gurtov. TCP performance in the presence of congestion and corruption losses. Master's thesis, University of Helsinki, Department of Computer Science, Helsinki, December 2000.

[Hay97] C. Hayes. Analyzing the performance of new TCP extensions over satellite links. Master's thesis, Ohio University, August 1997.

[HK97] T. Henderson and R. Katz. Satellite transport protocol (STP): An SSCOP-based transport protocol for datagram satellite networks. In Proceedings of 2nd Workshop on Satellite-Based Information Systems, 1997.

[HP91] N. C. Hutchinson and L. L. Peterson. The x-Kernel: An architecture for implementing network protocols. IEEE Transactions on Software Engineering, 17(1):64-76, January 1991.

[HV99] G. Holland and N. Vaidya. Analysis of TCP performance over mobile ad hoc networks. In Proceedings of IEEE/ACM MOBICOM '99, pages 219-230, August 1999.

[Jac88] V. Jacobson. Congestion avoidance and control. volume 18, pages 314329, Stanford, CA, August 1988.

[JB88] V. Jacobson and R. Braden. TCP extensions for long-delay paths. RFC 1072, October 1988.

[JBB92] V. Jacobson, R. Braden, and D. Borman. TCP extensions for high performance. RFC 1323, May 1992.

[Kat99] R. Katz. Satellite Transport Protocol. PhD thesis, December 1999. 
[KLB99] T. Kim, S. Lu, and V. Bharghavan. Improving congestion control performance through loss differentiation. Eighth International Conference on Computer Communications and Networks, October 1999.

[Koh96] D. Kohn. The Teledesic network: Using low-earth-orbit satellites to provide broadband, wireless, real-time internet access worldwide. http://www.isoc.org/isoc/whatis/conferences/inet/96/proceedings/g1/g1_3.htm, 1996.

[Lan01] D. Lane. HyperStat Online. http://davidmlane.com/hyperstat/index.html, 2001.

[LT01] A. Lahanas and V. Tsaoussidis. Behavior of TCP-probing with handoffs. In Proc. of IC'2001, volume 1, pages 325-331, 2001.

[Lud99] R. Ludwig. A case for flow-adaptive wireless links. Technical report, University of California, Berkeley, 1999.

[PA00] V. Paxson and M. Allman. Computing TCP's retransmission timer. RFC 2988, November 2000.

[PN98] K. Poduri and K. Nichols. Simulation studies of increased initial TCP window size. RFC 2415, September 1998.

[Pos81] J. Postel. Transmission control protocol. RFC 793, 1981.

[RJS02] K. Rubio, J. Janicik, and J. Szielenski. Chipsat's TCP/IP mission operations architecture - elegantly simple. In Proceedings of 16th Annual USU Conference on Small Satellites. SpaceDev, 2002. SSC02-IV-4.

[RS96] S. Ramanathan and M. Steenstrup. A survey of routing techniques for mobile communications networks. Mobile Networks and Applications, 1(2):89-104, 1996. 
[Sam99] N. Samaraweera. Non-congestion packet loss detection for TCP error recovery using wireless links. In IEEE Proceedings of Communications, volume 146, pages 222-230, August 1999.

[San99] R. Sangal. Performance analysis of the transmission control protocol over low earth orbit satellite communication systems. Master's thesis, College of Engineering and Technology, Ohio University, August 1999.

[SCP98] Space communication protocol specification (SCPS): Rationale, requirements and application notes. Technical report, Consultative Committee for Space Data Systems (CCSDS), August 1998. Green Book, Draft 0.4.

[SMM98] J. Semlie, J. Mahdavi, and M. Mathis. Automatic TCP buffer tuning. In Proceedings of ACM SIGCOMM'98, pages 315-323, 1998.

[SP98] T. Shepard and C. Partridge. When TCP starts up with four packets into only three buffers. RFC 2416, September 1998.

[SSC96] W. Sun, M. Sweeting, and A. Curiel. LEO satellite constellation for regional communication. Technical report, Center for Satellite Engineering Research, University of Surrey, UK, 1996.

[Sta00] W. Stallings. Data and Computer Communications. Prentice-Hall, sixth edition, 2000.

[Ste94] W. Stevens. TCP/IP Illustrated, Volume I; The Protocols. AddisonWesley, 1994.

[Ste96] W. Stevens. TCP/IP Illustrated, Volume III; TCP for Transactions, HTTP, NNTP, and the UNIX Domain Protocols. Addison-Wesley, 1996.

[Tan96] A. Tanenbaum. Computer Networks. Prentice-Hall International, 1996. 
[TB00] V. Tsaoussidis and H. Badr. TCP-probing: Towards an error control schema with energy and throughput performance gains. In Proceedings of the 8th IEEE Conference on Network Protocols, Japan, November 2000.

[TBGP00] V. Tsaoussidis, H. Badr, X. Ge, and K. Pentikousis. Energy / throughput tradeoffs of TCP error control strategies. In Proceedings of the 5th IEEE Symposium on Computers and Communications (ISCC), 2000.

[TBV00] V. Tsaoussidis, H. Badr, and R. Verma. Wave \& wait protocol (WWP): An energy-saving transport protocol for mobile IP-devices. In Proceedings of the 8th IEEE conference on networks, pages 469-473, September 2000.

[TM01] V. Tsaoussidis and I. Matta. Open issues on TCP for mobile computing. Technical Report 2001-013, 32001.

$\left[\mathrm{TTM}^{+}\right.$00] Y. Tobe, Y. Tamura, A. Molano, S. Ghosh, and H. Tokuda. Achieving moderate fairness for UDP flows by path-status classification. In 25th Annual IEEE Conference on Local Computer Networks (LCN'00), Tampa, Florida, November 2000.

[Vai99] N. Vaidya. Tutorial on TCP for wireless and mobile hosts, 1999.

[VH97] V. Visweswaraiah and J. Heidemann. Improving restart of idle TCP connections. Technical Report 97-661, University of Southern California, November 1997.

[Zha02] S. Zhang. Channel management, network representation and event handling of a protocol implementation framework for Linux using generative programming. Master's thesis, School of Computer Science, Carleto University, June 2002.

[ZT01] C. Zhang and V. Tsaoussidis. TCP real: Improving real-time capabilities of TCP over heterogeneous networks. In Proceedings of the 11th IEEE/ACM NOSSDAV, New York, 2001. 


\section{Appendix A}

\section{Transmission Control Protocol}

The Transmission Control Protocol (TCP) [Pos81, Bra89, Ste94] is the transport protocol of choice in the Internet today. It provides end-to-end connection-oriented fully reliable (complete, correct, in-sequence and without duplication) transport service on top of the Internet Protocol (IP). The protocol serializes user-data to the network in segments, whose size is bound by the Maximum Segment Size (MSS). This size is negotiated by the two ends of a connection during its establishment in a process known as the three-way handshake. A client typically starts by sending a SYN segment to a server (active-open request). The server replies to this segment by sending back its SYN-ACK segment, acknowledging the client's SYN and forwarding its own (passive-open request). The client then replies to that by sending an ACK segment, acknowledging the server's SYN. This process marks the start of the data transmission phase, where the client and the server transmit data segments to each other. When data transmission is completed, a four-way connection termination handshake 
is started, where each side sends its FIN segment and gets back a FIN-ACK.

As explained by Allman et al. in [APS99], TCP assigns a unique sequence number to each byte. Receivers acknowledge receiving segments by sending their own acknowledgement segments (ACKs). The protocol uses cumulative acknowledgements, where each ACK confirms arrival of all bytes up to the mentioned sequence number. Some segments with higher sequence numbers could have also been received. However, standard TCP has no mechanism to confirm that event. Moreover, the protocol manages flow control through a property called self-clocking. In the steady state, each arriving ACK triggers a transmission of a new segment. Receivers can delay acknowledging an arriving segment for a period of time, hoping for a chance to piggyback its ACK with a data segment transmitted in the same direction. Meanwhile should another segment arrive, an ACK is rushed right away acknowledging both segments together. On their part, TCP senders use an algorithm called Nagle's algorithm to delay sending small segments for some time until previous acknowledgements are received. This algorithm helps reduce the transmission overhead by minimizing the transmission of such small segments.

TCP segments are transmitted as IP datagrams, hence can be lost, reordered or duplicated. Receivers can discover such cases when they detect a sequence gap in the flow of arriving segments. Upon receiving "out-of-sequence" segments, receivers send back duplicate acknowledgments (DUPACK), which repeat the same sequence 
number as the last non-duplicate ACK. According to Paxson and Allman [PA00], a TCP sender maintains a smoothed round trip time (RTT) estimate. During the course of a connection, the sender frequently times transmitted segments until their corresponding ACKs arrive. When the segments arrive, the sender updates its RTT estimate with the newly measured round trip time. The sender uses this estimate as a retransmission timeout ( $\mathrm{RTO}$ ) that expires on transmitted segments when their corresponding ACKs fail to arrive on time. This timeout is exponentially backedoff with every unsuccessful retransmission of a segment. Having cached "out-ofsequence" segments, TCP receivers wait for the missing segments to arrive before passing them up to the application. Obviously, ACKs and timeouts are TCP's main tools for reliable data delivery.

TCP manages flow control by using a classic sliding window algorithm, as the one discussed by Tanenbaum in [Tan96]. By advertising a receiver window or rwnd with every ACK, TCP receivers cap the amount of data, which senders are allowed to transmit. An arriving ACK can either repeat the same window advertisement, increase it by advancing its right edge further to the right, or reduce it by advancing its left edge to the right. When the size of the outstanding (in flight) segments reaches the limit of the receiver window, further transmissions are blocked. According to Jacobson [Jac88], TCP sessions can force their peers to stop transmitting by advertising a zero-window. When this happens, the sender sessions enter a persist mode. In this 
mode, the sender sessions periodically probe their peers to force them to send window updates.

While the previous form of flow control is receiver-driven and aimed at preventing a fast sender from overwhelming a slow receiver, TCP employs another sender-driven flow control mechanism called congestion control. This mechanism, detailed by Allman et al. [APS99], is sensitive to the network's available capacity, and therefore prevents senders from overloading the network and eventually driving it to a state of congestive collapse. The mechanism has two phases: the slow start and the congestion avoidance. TCP senders try to estimate the network's available capacity by maintaining a congestion window (cwnd). At any point of time, the maximum number of segments allowed to be outstanding in the network swnd is governed by the following formula:

$$
\text { swnd }=\max (\text { cwnd }, \text { rwnd })
$$

Senders initialize their cwnd with an initial window of segments. During slow start, senders inflate cwnd by one segment for each arriving ACK, roughly doubling it every RTT (depending on whether receivers use delayed-acknowledgements). This exponential growth of $c w n d$ continues until a loss is detected or cwnd reaches the slow start threshold (ssthresh). At this point, senders switch to congestion avoidance, where they apply a linear increase to cwnd. During this phase, cwnd increases by one segment for each cwnd number of ACKs, or roughly one segment per RTT. Figure 


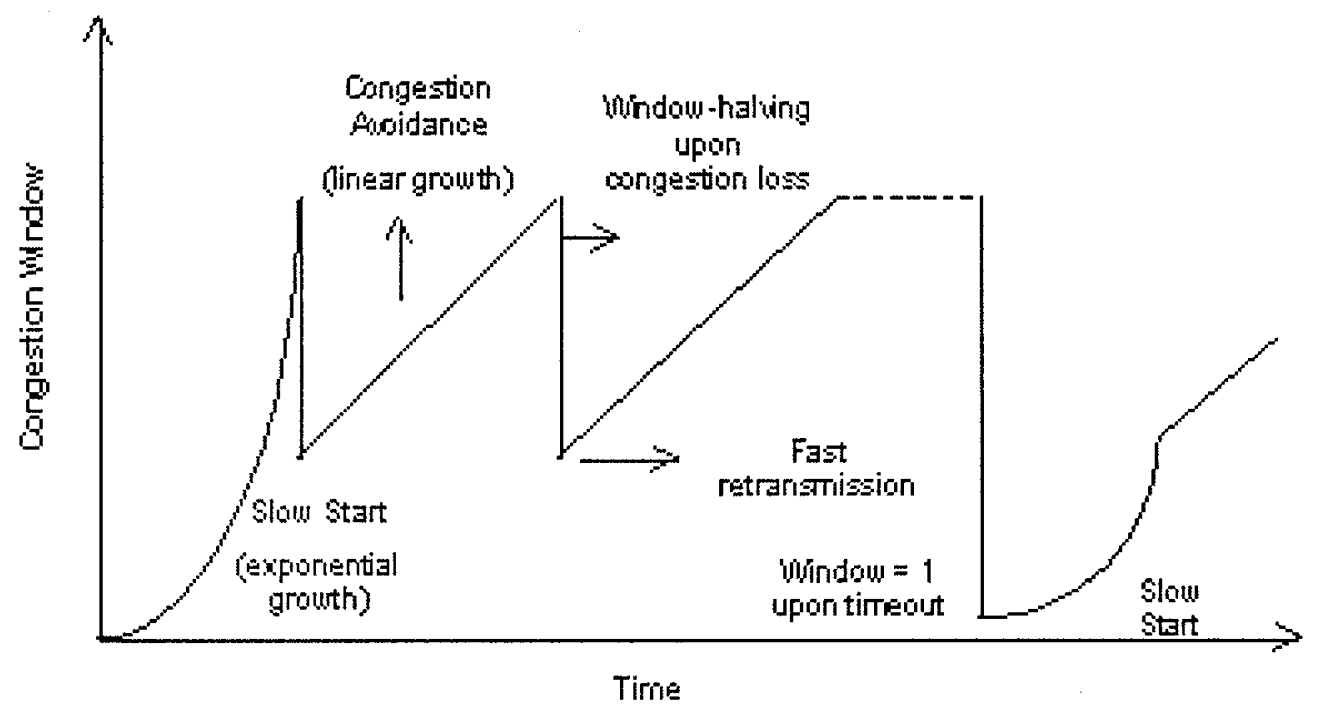

Figure A.1: A typical behavior of a TCP session [Gur00]

A.1 shows typical behavior of a TCP session. The following formula updates cwnd:

If cwnd $\leq$ ssthresh then

$$
c w n d=c w n d+M S S
$$

else

$$
c w n d=c w n d+\frac{M S S}{c w n d}
$$

TCP senders consider loss detection a sign of congestion and therefore react by slowing down their transmission rate. They achieve the slowdown by setting ssthresh to half the current swnd. Further, if the loss is detected by a retransmission timeout, the senders back-off their retransmission timer and reduce their cwnd to one segment, effectively re-entering slow start. This basic algorithm was enacted by an early 
version of TCP called Tahoe. However, to avoid waiting for a timeout to discover the loss, TCP was later enhanced by the fast retransmit and the fast recovery algorithms in a new version called Reno. As detailed in [APS99, Ste94], these algorithms make use of DUPACKs sent by receivers in response to out-of-order segments. Since some networks may re-order segments, these algorithms wait for a threshold of three DUPACKs before concluding that a loss is detected. This threshold was suggested as a compromise between bogus loss inference and timely recovery. When the threshold is reached, the DUPACKed segment is considered missing and therefore, is retransmitted. In addition, cwnd gets halved as a congestion control measure. Now the fast recovery algorithm is controlling the new data transmission until a non-duplicate ACK is received. The algorithm works on the assumption that every arriving DUPACK is an indication that a new segment has left the network, and therefore it can inflate cwnd temporarily by one more segment for each arriving DUPACK. When cwnd gets inflated beyond the current flight size, new segments start getting transmitted, effectively preserving the ACK clock. Once a non-duplicate ACK arrives, the algorithm is terminated and cwnd is deflated.

Reno is most efficient when a single segment is lost. However, when multiple segments are lost, timeouts occur, which make its fast recovery algorithm less useful. NewReno, presented by Floyd and Henderson in [FH99], is another variation of TCP that can recover from multiple losses at a rate of one loss per RTT. The recovery 
phase here does not end until all segments prior to the fast retransmit phase are acknowledged by a non-duplicate ACK. Therefore, if such an ACK only partially acknowledges those segments, it is taken as an indication that more segments are lost in the same window. In this case, the presumed segments are retransmitted and the recovery phase is prolonged until the precondition is met or the retransmission timer expires. 


\section{Appendix B}

\section{Measurement Data}

The following tables show the complete results of the simulation tests for the three different satellite error categories. All time results are approximated to the nearest second. The effective throughput and the throughput/overhead ratio results are rounded to the nearest integer. For the overhead and effective throughput measurements, the standard deviation and the $95 \%$ confidence interval are also calculated for each statistic. The standard deviation (SD) $\sigma$ is calculated by the following formula ${ }^{1}[\operatorname{Lan} 01]:$

$$
\sigma=\sqrt[2]{\frac{\sum_{k=1}^{n}\left(X_{k}-\bar{X}\right)^{2}}{n-1}}
$$

where $n$ is the number of replications, $\bar{X}$ is the mean value and $X_{k}$ is the $\mathrm{k}$ th value. The confidence interval $C I$ is calculated by the following formula ${ }^{2}$ [Lan01]:

$$
C I=\bar{X} \pm Z \times \frac{\sigma}{\sqrt[2]{n}}
$$

\footnotetext{
${ }^{1}$ This formula calculates the unbiased value of the standard deviation.

${ }^{2}$ This formula is customized for a $95 \%$ confidence interval by setting $Z$ to 1.96 .
} 
where $n$ is the number of replications, $\bar{X}$ is the mean value, $\sigma$ is the standard deviation and $Z$ is a statistical value corresponding to the desired confidence interval. 


\begin{tabular}{|c|c|c|c|c|}
\hline 을을을 & 另另另穷另另另 & 999999 & 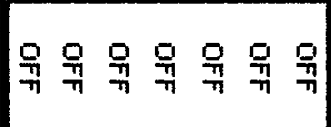 & \\
\hline 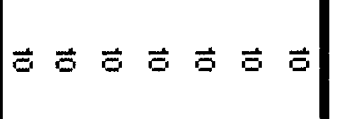 & 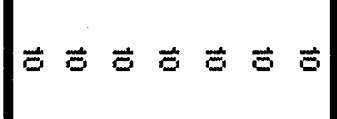 & - & & h \\
\hline-0 & $8 \mathrm{~g}=-$ & $8080 m-0$ & $0 \& 8=\omega-0$ & $H \frac{1}{n}$ \\
\hline 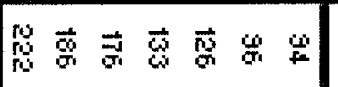 & 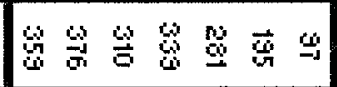 & 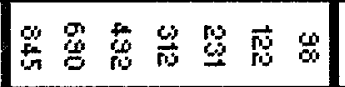 & 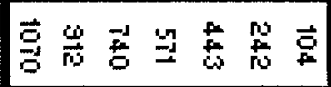 & \\
\hline 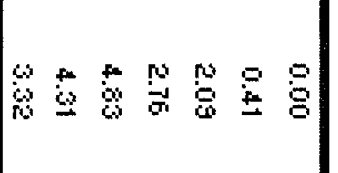 & $\vec{s}$ & 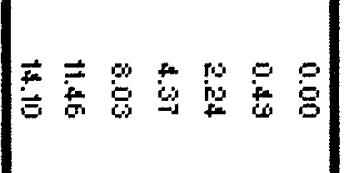 & 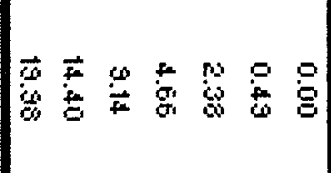 & $\frac{5}{6} \frac{1}{6}$ \\
\hline $\overrightarrow{8}$ & $\overrightarrow{\mathrm{G}} \overrightarrow{\mathrm{g}}$ & 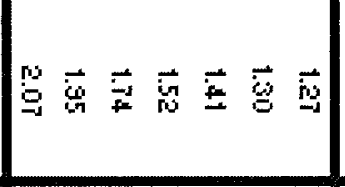 & 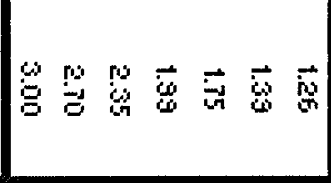 & $\begin{array}{l}0 \\
\frac{1}{6} \\
4 \\
\frac{8}{4}\end{array}$ \\
\hline 0 & 9899898 & 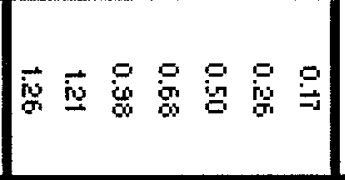 & 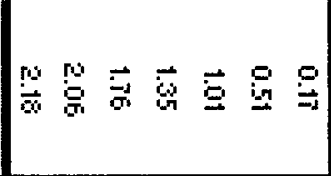 & $\frac{5}{6} \frac{8}{6}$ \\
\hline 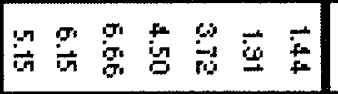 & 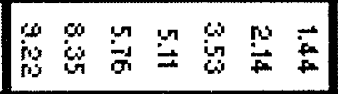 & 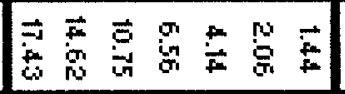 & 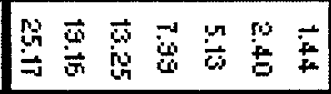 & $\frac{1}{t}$ \\
\hline 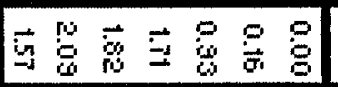 & $\mathrm{g}$ & $\overrightarrow{8} 8 \mathrm{~g} 8 \mathrm{~g}$ & 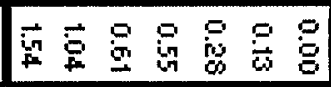 & 6 \\
\hline 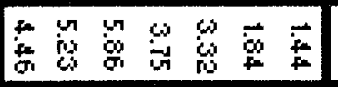 & 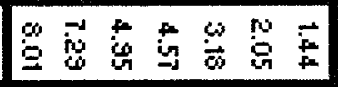 & 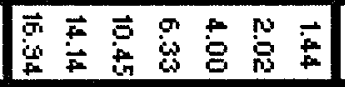 & 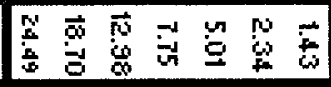 & 4 \\
\hline 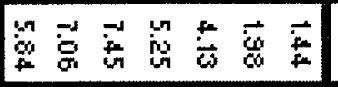 & 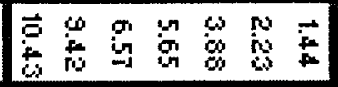 & $\overrightarrow{\dot{\theta}}$ & 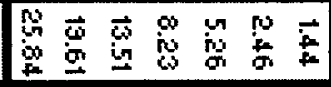 & 8 \\
\hline 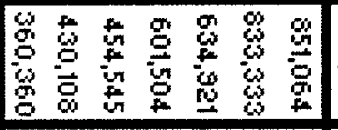 & 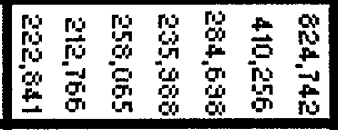 & 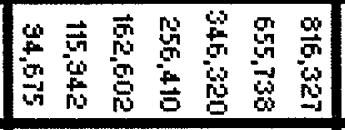 & 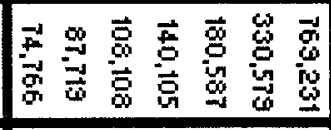 & $\frac{1}{8}$ \\
\hline 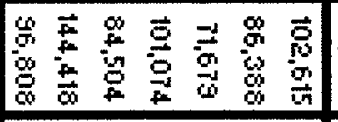 & 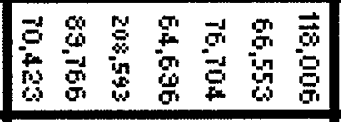 & 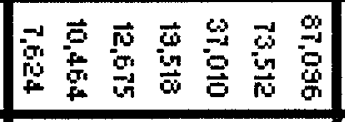 & 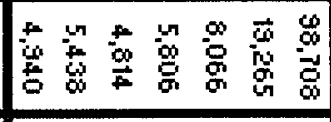 & 6 \\
\hline 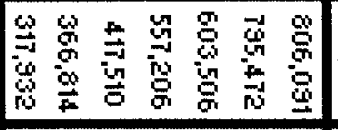 & 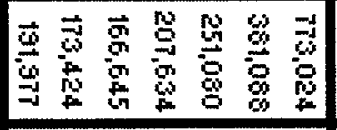 & 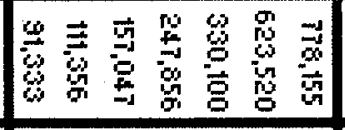 & 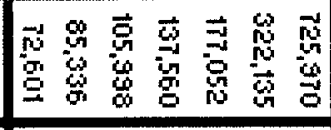 & +8 \\
\hline 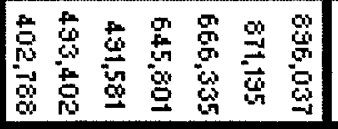 & 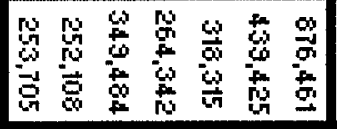 & 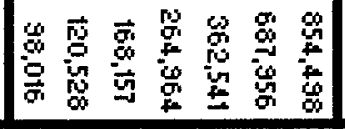 & 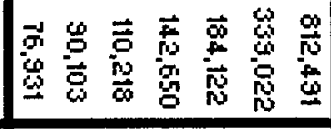 & 8 \\
\hline 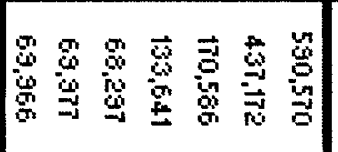 & 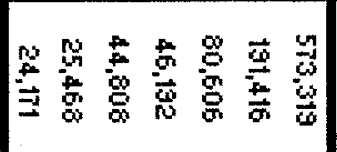 & 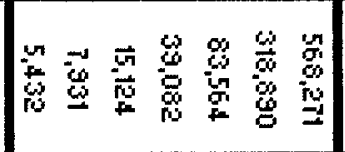 & 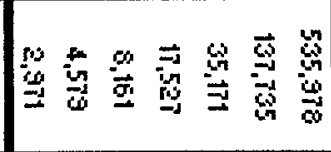 & 5 \\
\hline
\end{tabular}

Table B.1: Bit Corruption Test Measurements 


\begin{tabular}{|c|c|c|c|c|}
\hline 9999 & 䎏号界界易 & 올을울 & 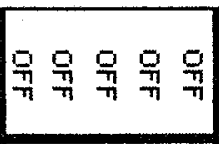 & \\
\hline & & $\therefore 8$ & 8989 & in \\
\hline$\cdots-1$ & - & $\omega-$ & & \\
\hline$\Leftrightarrow \Xi$ & $\exists \overrightarrow{B^{\prime}}$ & 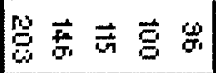 & 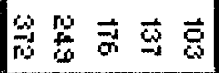 & \%: \\
\hline 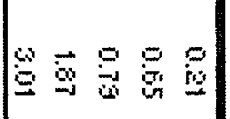 & 68 & $\frac{b}{2} \overrightarrow{8}$ & 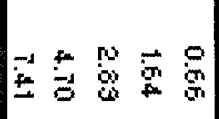 & $\frac{2}{7}$ \\
\hline$\vec{Q} \overrightarrow{8} \overrightarrow{8}$ & $\overrightarrow{\mathrm{g}} \overrightarrow{\mathrm{g}} \overrightarrow{\mathrm{g}} \overrightarrow{\mathrm{n}}$ & & $\vec{G} \vec{G} \vec{Q}$ & $+\frac{1}{2} \frac{6}{8}$ \\
\hline $89 \frac{9}{9} 9$ & 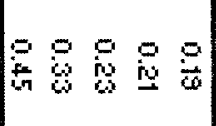 & $\frac{g}{9}$ & 8989 & $m \frac{1}{6}$ \\
\hline 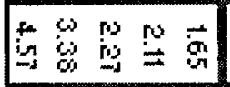 & 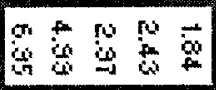 & 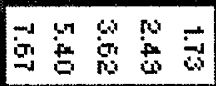 & 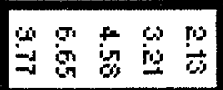 & \\
\hline $89 \begin{array}{ll}9 \\
0\end{array}$ & $\vec{\sigma} 989$ & 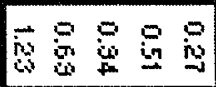 & 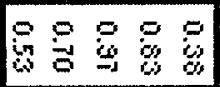 & \\
\hline 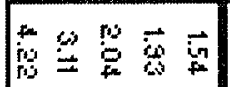 & 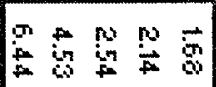 & 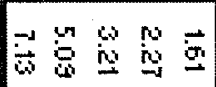 & $\begin{array}{l}\infty \\
0\end{array}$ & \\
\hline 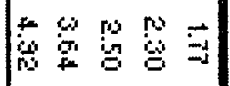 & 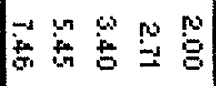 & $\begin{array}{l}\infty \\
\mathrm{B} \\
\mathrm{B}\end{array}$ & 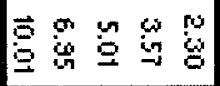 & 95 \\
\hline 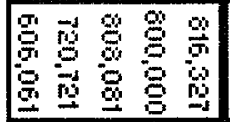 & 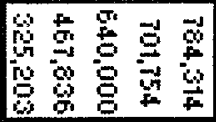 & 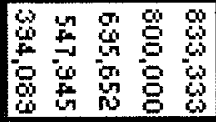 & 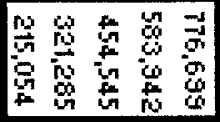 & $\frac{\mu}{\sigma}$ \\
\hline 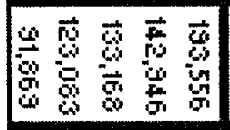 & 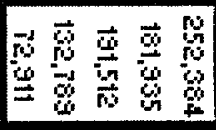 & 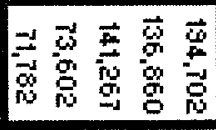 & 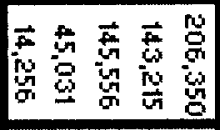 & 8 \\
\hline 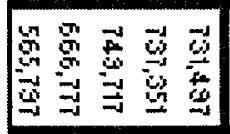 & 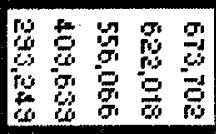 & 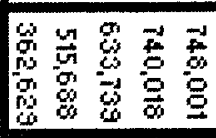 & 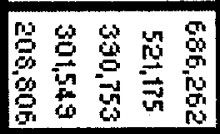 & 8 \\
\hline 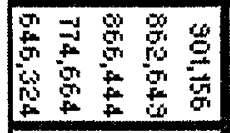 & 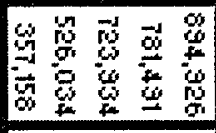 & 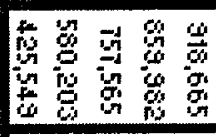 & 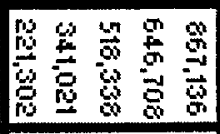 & 8 \\
\hline 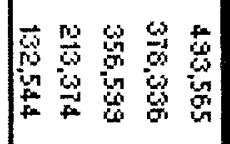 & 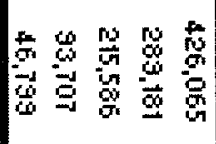 & 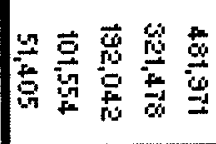 & 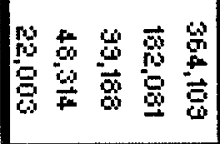 & 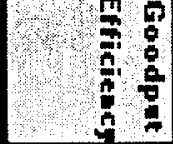 \\
\hline
\end{tabular}

Table B.2: Handoff Test Measurements 


\begin{tabular}{|c|c|c|c|c|}
\hline 99999 & 另号号另另 & 몰몰을 & 易另另另易 & 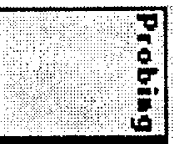 \\
\hline$\dot{\theta} \theta \dot{\theta} \bar{\theta}$ & 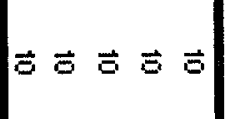 & & & ${ }_{n}^{4}:$ \\
\hline$\vec{\theta}$ & $\Theta \mathrm{E} \quad \overrightarrow{\mathrm{s}}$ & $\Theta g$ & & $+\frac{3}{4}$ \\
\hline 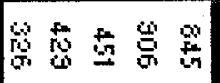 & 96898 & 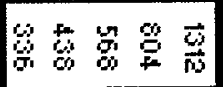 & 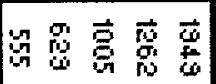 & in \\
\hline 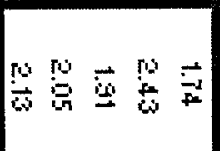 & 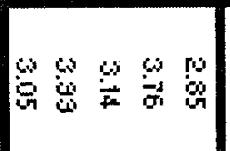 & 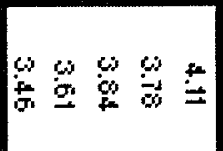 & 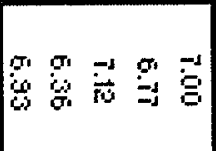 & 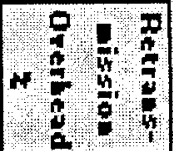 \\
\hline$\vec{y}$ & $\overrightarrow{\mathrm{g}} \overrightarrow{\mathrm{g}} \overrightarrow{\mathrm{g}} \overrightarrow{\mathrm{b}}$ & $\vec{f} \vec{g} \vec{g}$ & $\overrightarrow{3} \vec{b} \vec{b}$ & 8 \\
\hline 8080 & $88 \mathrm{~B} 8$ & 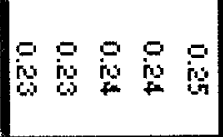 & 80898 & $4 \frac{6}{6} 8 \frac{6}{8}$ \\
\hline $\begin{array}{l}0 \\
\partial+2\end{array}$ & 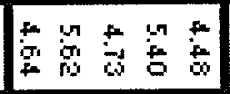 & 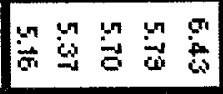 & 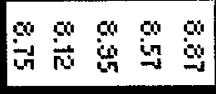 & $\frac{5}{5}$ \\
\hline $\overrightarrow{\mathrm{B}} \mathrm{g} \dot{\mathrm{g}} \mathrm{g}$ & $\overrightarrow{\mathrm{s}} \overrightarrow{\mathrm{g}} \overrightarrow{\mathrm{B}}$ & 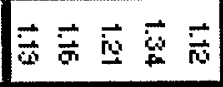 & $\overrightarrow{8}$ & \\
\hline$\Leftrightarrow$ & 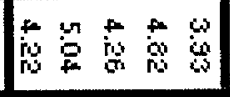 & 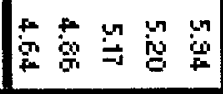 & 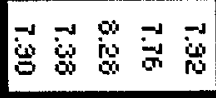 & $\theta$ 象 \\
\hline 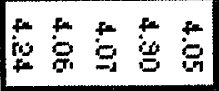 & $\Leftrightarrow \frac{\pi}{a} \mathrm{~g}$ & 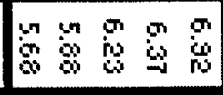 & 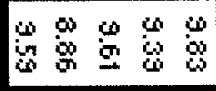 & $\mathbf{E}^{2}$ \\
\hline 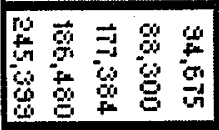 & 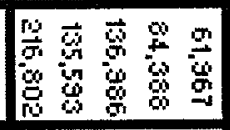 & 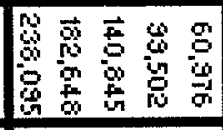 & 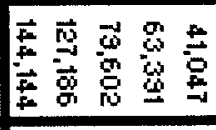 & $\frac{4}{4}$ \\
\hline 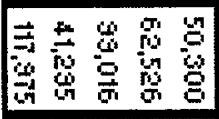 & 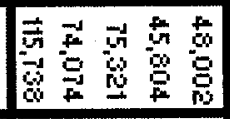 & 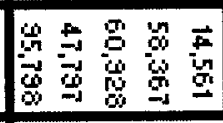 & 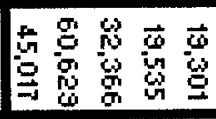 & 6 \\
\hline 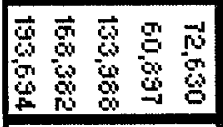 & 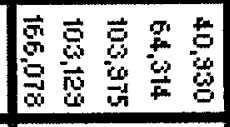 & 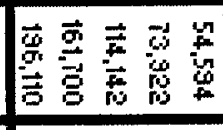 & 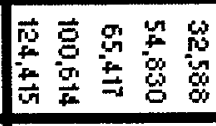 & H \\
\hline 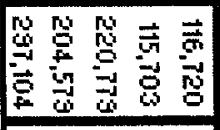 & 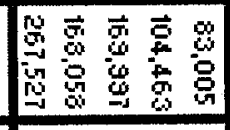 & 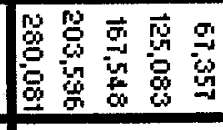 & 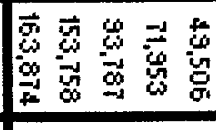 & 8 \\
\hline 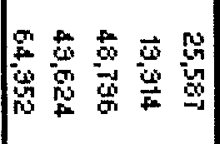 & 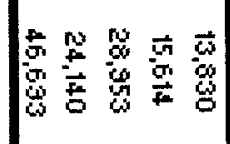 & 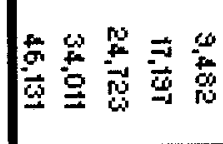 & 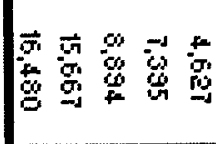 & \\
\hline
\end{tabular}

Table B.3: Limited Connectivity Test Measurements 